\title{
Advances in Passive Acoustic Detection, Localization, and Tracking Applied to Unmanned Underwater Vehicles
}

\author{
by \\ Kristen Railey Kita
}

S.B. Mechanical Engineering, Massachusetts Institute of Technology (2013)

S.M. Mechanical Engineering, Massachusetts Institute of Technology (2018)

Submitted to the Department of Mechanical Engineering

in partial fulfillment of the requirements for the degree of

Doctor of Philosophy

at the

MASSACHUSETTS INSTITUTE OF TECHNOLOGY

and the

WOODS HOLE OCEANOGRAPHIC INSTITUTION

February 2022

(c) 2022 Kristen Railey Kita. All rights reserved.

The author hereby grants to MIT, WHOI, and The Charles Stark Draper Laboratory, Inc. permission to reproduce and to distribute publicly paper and electronic copies of this thesis document in whole or in part in any medium now known or hereafter created.

Author

Department of Mechanical Engineering, MIT

Applied Ocean Science \& Engineering, WHOI

October 26, 2021

Certified by

Henrik Schmidt

Professor of Mechanical and Ocean Engineering, MIT

Thesis Supervisor

Certified by . .

Dino DiBiaso

Distinguished Member of Technical Staff, The Charles Stark Draper Laboratory, Inc.

Thesis Supervisor

Accepted by

Nicolas Hadjiconstantinou

Professor of Mechanical Engineering, MIT

Chair, Department Committee for Graduate Students

Accepted by

David Ralston

Associate Scientist with Tenure, WHOI

Chair, Joint Committee for Applied Ocean Science \& Engineering 


\title{
Advances in Passive Acoustic Detection, Localization, and Tracking Applied to Unmanned Underwater Vehicles
}

\author{
by \\ Kristen Railey Kita \\ Submitted to the Department of Mechanical Engineering \\ on October 26, 2021, in partial fulfillment of the \\ requirements for the degree of \\ Doctor of Philosophy
}

\begin{abstract}
Detection, classification, localization, and tracking (DCLT) of unmanned underwater vehicles (UUVs) in the presence of shipping traffic is a critical task for passive acoustic harbor security systems. In general, vessels can be tracked by their unique acoustic signature due to machinery vibration and cavitation noise. However, cavitation noise of UUVs is considerably quieter than ships and boats, making detection significantly more challenging. In this thesis, I demonstrated that it is possible to passively track a UUV from its highfrequency motor noise using a stationary array in shallow-water experiments with passing boats. First, causes of high frequency tones were determined through direct measurements of two UUVs at a range of speeds. From this analysis, common and dominant features of noise were established: strong tones at the motor's pulse-width modulated frequency and its harmonics. From the unique acoustic signature of the motor, I derived a high-precision, remote sensing method for estimating propeller rotation rate. In shallow-water UUV field experiments, I demonstrated that detecting a UUV from motor noise, in comparison to broadband noise from the vehicle, reduces false alarms from $45 \%$ to $8.4 \%$ for $90 \%$ true detections. Beamforming on the motor noise, in comparison to broadband noise, improved the bearing accuracy by a factor of $3.2 \times$. Because the signal is also high-frequency, the Doppler effect on motor noise is observable and I demonstrate that range rate can be measured. Furthermore, measuring motor noise was a superior method to the "detection of envelope modulation on noise" algorithm for estimating the propeller rotation rate. Extrapolating multiple measurements from the motor signature is significant because Bearing-DopplerRPM measurements outperform traditional bearing-Doppler target motion analysis. In the unscented Kalman filter implementation, the tracking solution accuracy for bearing, bearing rate, range, and range rate improved by a factor $2.2 \times, 15.8 \times, 3.1 \times$, and $6.2 \times$ respectively. These findings are significant for improving UUV localization and tracking, and for informing the next-generation of quiet UUV propulsion systems.
\end{abstract}

Thesis Supervisor: Henrik Schmidt

Title: Professor of Mechanical and Ocean Engineering, MIT

Thesis Supervisor: Dino DiBiaso

Title: Distinguished Member of Technical Staff, The Charles Stark Draper Laboratory, Inc. 


\section{Acknowledgments}

First and foremost, I would like to thank my advisors Henrik Schmidt and Dino DiBiaso for your support, guidance, and confidence in me to become a more skilled and independent researcher. Thank you to Henrik, your curiosity and passion for ocean acoustics and robotics inspires me. I appreciate your optimism and encouragement to try new things and learn from past experiments. Thank you to Dino, your knowledge of robotics, systems engineering, and Navy interests was so helpful in guiding my research. I am also grateful for your expertise and assistance with the Riptide UUV field tests, which led to some of the key findings of this thesis work.

Thank you to my committee members and thesis chair - Arthur Baggeroer, Dana Yoerger, John Leonard, and Andone Lavery- for your suggestions, feedback, and questions that strengthened this thesis work.

Thank you to my fellow LAMSS members, alumni, and collaborators for your friendship, interesting technical discussions, and support over the years: Rui Chen, Blake Cole, Oscar Viquez, Nick Rypkema, Supun Randeni, Greg Nannig, Paul Robinette, Michael Novitzky, Erin Fischell, Mike Benjamin, Scott Carper, Dan Goodwin, Bradli Howard, Craig Evans, Caileigh Fitzgerald, Brendan O’Neill, Kyle Woerner, and Eeshan Bhatt.

Thank you to Joel Parry for your mentorship and feedback on this work. Thank you to my mentors Doug Hart, Joe Edwards, and Nick Pulsone for introducing me to the exciting field of unmanned underwater vehicles when I was an undergrad at MIT taking 2.013/2.014.

I am so grateful for the support of my friends that I met or have gotten to know better through grad school. Special thank you to Janille Maragh, Jackie Ohmura, Uyanga Tsedev, Sara Nagelberg, and Jean Sack. Thank you to the "Mega"women Hannah Varner, Rebecca Zubajlo, Andrea Lehn, Nastasia Winey, Miranda Kotidis, and Katie Cavanagh.

Thank you to all my friends near and far for your support and encouragement, especially Lauren Ciaravalli. Thank you to Veronica Lopez, Danielle Class, Elina Hu, Valerie Anderson, Sascha Burton, Ola Kalinowska, Alix de Monts, Jackie Sly, Rebecca Yeh, Amanda Rivamonte, Patrick Nginyah, Victoria McCrave, and Bibi Koch.

I am so grateful to my parents, Cheryl and Malcolm, for always believing in me and encouraging me to follow my passion. Thank you to my brothers, Stuart and Owen, for your love, support, and humor. 
Thank you to my in-laws, Betsy, Mark, Ashley, and Raf for welcoming me into your family, and giving me encouragement and support during my $\mathrm{PhD}$.

Lastly, thank you to my husband and best friend, Derek. I am so grateful for all the love and support you have given me during the $\mathrm{PhD}$ journey. I will always cherish the memories we had together as grad students, and I am so excited for what adventures come next.

I am grateful for the financial support for this research from the Draper Scholarship, the National Defense Science and Engineering Graduate Fellowship, the Office of Naval Research, and DARPA. Chapter 3 of this thesis is reproduced from: Railey, Kristen, Dino DiBiaso, and Henrik Schmidt. "An acoustic remote sensing method for high-precision propeller rotation and speed estimation of unmanned underwater vehicles." The Journal of the Acoustical Society of America 148.6 (2020): 3942-3950 with the permission of AIP Publishing. 


\section{Contents}

1 Introduction $\quad 21$

1.1 Commercial and Research Applications for UUVs . . . . . . . . . . . . 22

1.2 The Role of UUVs in the Modern Navy . . . . . . . . . . . . . . 23

1.3 The Counter-UUV Problem . . . . . . . . . . . . . . . . . . . 24

1.4 Summary of Contributions . . . . . . . . . . . . . . . . 26

1.5 Thesis Organization . . . . . . . . . . . . . . 27

2 Background $\quad 29$

2.1 Unmanned Underwater Vehicles . . . . . . . . . . . . . . . . . . . 29

2.1 .1 Navigation . . . . . . . . . . . . . . . . . . . 29

2.1.2 Control and Propulsion Systems _. . . . . . . . . . . . 32

2.1.3 Low-cost, Micro-UUV Design . . . . . . . . . . . . . . . . 33

2.1.4 Sandshark UUV . . . . . . . . . . . . . . . . . . . . 34

2.1 .5 Riptide UUV . . . . . . . . . . . . . . . . . 35

2.2 Radiated Acoustic Noise of Vessels . . . . . . . . . . . . . . . . . . 36

2.3 Permanent Magnetic AC Synchronous Motors . . . . . . . . . . . . 38

2.3 .1 Torque Ripple . . . . . . . . . . . . . . . . . . 39

2.3 .2 Cogging Torque . . . . . . . . . . . . . . . . . . 41

2.3.3 Commutation Methods . . . . . . . . . . . . . 42

2.3.4 Sensorless Control Techniques . . . . . . . . . . . . . 43

2.3.5 Radial Electromagnetic Forces . . . . . . . . . . . . . . . . 44

2.4 Acoustic Array Design and Signal Processing Methods . . . . . . . . . . 45

2.4.1 Conventional Beamforming . . . . . . . . . . . . 45

2.4 .2 Array Design Parameters . . . . . . . . . . . . . . 50 
2.4.3 Frequency-domain Snapshot Model for Broadband Beamforming . . 52

2.4.4 Adaptive Beamforming . . . . . . . . . . . . . . . 52

2.4.5 Design of the Acoustic Array System . . . . . . . . . . . . 54

2.4.6 Effect of Temporal and Spatial Aliasing . . . . . . . . . . . . 54

2.5 Pool and Tank experiments . . . . . . . . . . . . . . . 56

2.6 Target Motion Analysis . . . . . . . . . . . . . . . . 58

2.6.1 Batch Processing Least-squares Estimation . . . . . . . . . . 60

2.6 .2 Kalman Filter . . . . . . . . . . . . . . . . . . . . . . . . 62

2.6.3 Unscented Kalman Filter _. . . . . . . . . . . . . . . . . . . 66

3 An Acoustic Remote Sensing Method for High-precision Propeller Ro$\begin{array}{ll}\text { tation and Speed Estimation of UUVs } & 71\end{array}$

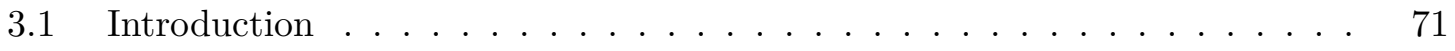

3.2 Methodology . . . . . . . . . . . . . . . . . . . . . 74

3.2.1 Overview of the Sandshark and Riptide Micro-UUVs . . . . . . . 74

3.2.2 Identifying UUV Acoustic Features . . . . . . . . . . . 75

3.2.3 Speed and Propeller Rotation Measurement Method . . . . . . . . . 76

3.2.4 Demonstration of Speed Estimation in Field Experiments . . . . . . 77

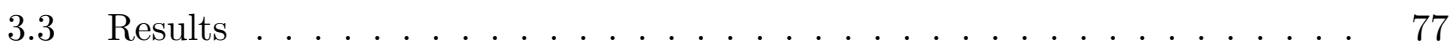

3.3 .1 Sandshark UUV Acoustic Signature . . . . . . . . . . . 77

3.3.2 Riptide UUV and T200 Thruster Acoustic Signature . . . . . . . 78

3.3.3 Field Measurements of UUV Acoustic Features . . . . . . . . . . . . 79

3.3.4 Derivation and Field Demonstration of Speed and Propeller Rotation Estimation Method . . . . . . . . . . . . 82

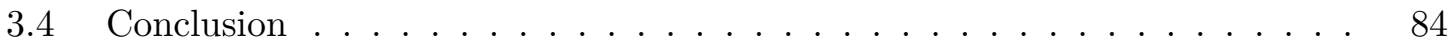

4 Passive Acoustic Detection and Tracking of a UUV from High-frequency $\begin{array}{ll}\text { Motor Noise } & \mathbf{8 9}\end{array}$

4.1 Introduction . . . . . . . . . . . . . . . 89

4.2 Methods . . . . . . . . . . . . . . . . . . . . . 95

4.2.1 Description of the Unmanned Underwater Vehicle . . . . . . . . 95

$4.2 .2 \quad$ Field Test Set-up . . . . . . . . . . . . . . . 95

4.2.3 Acoustic Array Data Analysis . . . . . . . . . . . . . . . 98 
4.2.4 Estimating the UUV Speed Prediction Model . . . . . . . . . . . 103

4.2.5 Unscented Kalman Filter Implementation . . . . . . . . . . . . . . . 104

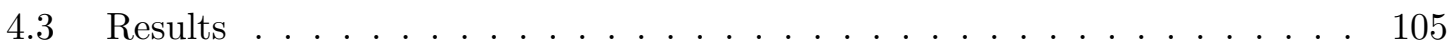

4.3.1 Passive Acoustic Detection . . . . . . . . . . . . . . 105

4.3.2 Direction of Arrival Estimation . . . . . . . . . . . . 106

4.3.3 Range Rate and RPM . . . . . . . . . . . . . . . . . . . . . 108

4.3.4 Localization and Tracking . . . . . . . . . . . . . 110

4.3.5 Robustness of RPM-Speed model . . . . . . . . . . . . . . 113

4.4 Conclusion . . . . . . . . . . . . . . . . . . . . 113

5 Conclusion $\quad 119$

5.1 Implementation in the Ocean Environment . . . . . . . . . . . . . 119

5.2 Range Limitations of UUV Detection from Motor Noise . . . . . . . . . . 121

5.3 Future Work . . . . . . . . . . . . . . . . . . . 123

5.4 Concluding Remarks . . . . . . . . . . . . . . . . . . . 126

A MPDR Beamforming Results 131

B UUV Source Level Estimates and Additional DEMON Algorithm Analysis

$\begin{array}{ll}\text { C Observability of Range } & 143\end{array}$ 


\section{List of Figures}

2-1 The Sandshark UUV is used in field testing in the Charles River of Boston,

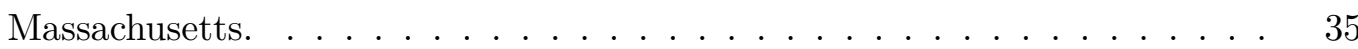

2-2 The Riptide UUV is tested in field experiments in the Charles River of Boston, Massachusetts. . . . . . . . . . . . . . . 36

2-3 Diagram of how machinery noise is generated inside a diesel-engine machine. Adapted from Urick et al. $[1] \ldots \ldots$. . . . . . . . . . . . . 37

2-4 Cavitation noise of submarines increases with higher speeds and lower depths. Adapted from Urick et al. $[1] \ldots \ldots$. . . . . . . . . . . . .

2-5 Image of the inside of a BLDC motor: (a) permanent magnets on the rotor (b) stator coils. . . . . . . . . . . . . . . . . . . 40

2-6 Diagram of the inside of a brushless DC motor. The rotor (the part that rotates) has permanent magnets which interact with the energized coils on the stator (stationary part). The rotor rotates sequentially as the coils are energized as shown from positions $(\mathrm{a})$ to $(\mathrm{b}) . \ldots \ldots \ldots$

2-7 The torque output (T), back-EMF (E), and current (I) are plotted for onephase. (a) Constant torque results from an ideal quasi-square wave. (b) Torque ripple occurs in reality because current can't instantaneously rise and fall. Image modified from $[2] \ldots \ldots \ldots$

2-8 Trapezoidal or six-step commutation for a brushless DC motor with Hall sensors is illustrated. . . . . . . . . . . . . . . . . . 43

$2-9$ Line array diagram $\ldots \ldots \ldots \ldots \ldots \ldots \ldots$

2-10 Delay-and-sum beamformer for an incoming plane wave $f(t) \ldots \ldots$. . . 48

2-11 Beampatterns are presented for $8000 \mathrm{~Hz}$ steered to (a) broadside and (c) 45 degrees, and $17500 \mathrm{~Hz}$ steered to (b) broadside and (d) 45 degrees. . . . . . 
2-12 Process for frequency-domain snapshot model for broadband beamforming .

2-13 The 8-channel data collection method for acoustic measurements is designed to compactly fit in a watertight electronics box. . . . . . . . . . 55

2-14 Array setup options . . . . . . . . . . . . . . . . . . 56

2-15 Pool experiment setups where the (a) Sandshark and (b) Riptide UUV were held in place on the left wall. On the other end of the pool, (c) an offboard horizontal line array collected acoustic measurements. . . . . . . . . .

2-16 (a) Diagram of the tank experiment for collecting acoustic noise from the thruster is presented. (b) The acoustic data is collected from two hydrophones. The thruster is mounted to the bottom of the tank. . . . . . . . . . 58

2-17 Kalman filter process, adapted from Bar-Shalom et al. $[3] \ldots \ldots 2$

3-1 During field experiments with the (a) Sandshark and (b) Riptide UUVs, acoustic data was collected from the MIT Sailing Pavilion in the Charles River. The vehicle position over time, plotted here, was logged by the IMUbased navigation system. . . . . . . . . . . . . . . . .

3-2 (a) The radiated noise from a Sandshark UUV was characterized at different speeds, followed by isolated fin movements. Three features are highlighted: (b) a constant tone at $19,201 \mathrm{~Hz}$, which matches $f_{\mathrm{PWM}}$ and sidebands that increase with speed; $(\mathrm{c})$ a tone at $2 f_{\mathrm{PWM} \text {,aliased }} \ldots \ldots \ldots$

3-3 The radiated noise of the Sandshark at $400 \mathrm{rpm}$ and the Riptide at approximately $870 \mathrm{rpm}(1 \mathrm{~m} / \mathrm{s}$ desired speed $)$ are characterized in plots (a)-(c) and (d)-(f) respectively. The PSD plots are centered at $f_{\mathrm{PWM}}$ and $2 f_{\mathrm{PWM} \text {,aliased }}$ which are: (a) $19,205 \mathrm{~Hz}$ (b) $21,650 \mathrm{~Hz}$ (d) $17,884 \mathrm{~Hz}$, and (e) 24,292 Hz. The DEMON spectrum is plotted in (c) and (f). Grey lines indicate $p f_{\mathrm{s}}$ and the

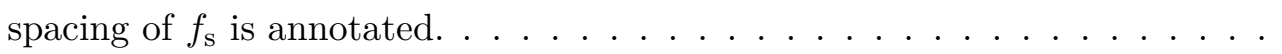

3-4 (a) A spectrogram of the Riptide UUV noise characterization test is centered at $f_{\mathrm{PWM}}$. (b) The DEMON spectrum is plotted below. Sideband spacings in (a) correspond to the tones in the $(\mathrm{b}) \ldots \ldots \ldots \ldots$

3-5 The spectrogram of the T200 motor isolation experiment is centered at $24,008 \mathrm{~Hz}\left(f_{\mathrm{PWM}}\right)$. As the speed increases, sidebands centered around the $f_{\mathrm{PWM}}$ carrier increase their interval spacing. . . . . . . . . . . 
3-6 (a) The spectrogram of acoustic data from the Sandshark noise experiment in the Charles River is compared to (b) the vehicle navigation data. The tone at $18,270 \mathrm{~Hz}$ with sideband spacing at $142 \mathrm{~Hz}$ and the tone at $17,504 \mathrm{~Hz}$ correlate to the PWM switching frequencies of the thruster motor and fin servomotors. The former tone is present when the vehicle is underway. . . .

3-7 (a) The spectrogram of acoustic data from the Riptide noise experiment in the Charles River is compared to (b) the DEMON spectrum, and (c) the vehicle navigation data from the INS of desired and actual speed. In (a), the constant tone at $17,890 \mathrm{~Hz}$ corresponds to $f_{\mathrm{PWM}}$ with sideband spacings of $f_{\mathrm{m}}$ at $212 \mathrm{~Hz}$, which increase to $251 \mathrm{~Hz}$ with a desired speed change. The values of $f_{\mathrm{m}}$ are also present in $(\mathrm{b}) \ldots \ldots \ldots \ldots$

3-8 The acoustic measurements of the sideband frequency intervals, $f_{m}$, are compared to the corresponding motor speeds, $f_{\mathrm{s}}$, for the (a) T200 thruster and (c) Sandshark UUV, and the desired speed for (b) the Riptide vehicle. The predicted sideband spacings are in dashed black. In (b) and (c) lines are drawn to show measured sideband spacings versus the true vehicle speed in the field experiments. . . . . . . . . . . . . . . . .

4-1 For the Charles River field experiment, the Sandshark vehicle, pictured in the corner, was deployed from the MIT sailing pavilion dock. Acoustic measurements of the radiating noise from the UUV were collected from the dockmounted array. The vehicle IMU-based navigation solution is plotted over time, shown in color (starting in blue, ending in red). There are three relevant coordinate systems shown here. First, a local cartesian coordinate system, where the origin is dock-centered. Second, the acoustic array polar coordinate system. Third, the coordinate system fixed to the UUV. . . . . .

4-2 (a) Speed and depth of the vehicle over time during the Charles River field experiment. (b) Distance travelled by the vehicle between each time step shows significant jumps when a GPS fix is acquired. . . . . . . . . . 
4-3 A spectrogram of the acoustic data collected in the Charles River field test experiment with radiating noise from the Sandshark UUV is presented. Strong tones from the motor inside the vehicle's propulsion system and broadband acoustic noise emitting from the vehicle are observable. During the experiment, boats passed by the robot, creating loud broadband interfering noise.

4-4 Signal processing chain for detection, localization, and tracking of the vehicle from motor noise. . . . . . . . . . . . . . . . . . . . .

4-5 ROC curve analysis is performed to compare detecting the UUV in the Charles River field test with two energy detectors. The first detector uses a bandpass filter on broadband noise and the second detector uses a bandpass filter on the motor signature noise. . . . . . . . . . . . .

4-6 Passive acoustic detections of the UUV are plotted over time with 1 corresponding to a detection and 0 corresponding to no detection. The UUV is present from 760-1920 seconds. Two energy detectors are compared with different bandpass filters for isolating broadband vehicle noise and the motor acoustic signature at (a) $1000-8000 \mathrm{~Hz}$ and (b) $17450-17550 \mathrm{~Hz}$ respectively. The threshold for both detectors is set at $90 \%$ probability of detection. . .

4-7 Considering only the period of the experiment where the UUV is present, the acoustic data collected on the radiated noise of the vehicle from the line array is analyzed. (a) The acoustic data from a single hydrophone is used to generate a spectrogram. A timeline of when boats are present is denoted in red to the left of the spectrogram. Bearing time recordings of the radiating acoustic noise of the UUV (b) broadband noise and (d) motor noise are compared. The respective direction of arrival plots are in (c) and (e). . . .

4-8 (a) The Doppler shift of the fin servomotor's PWM switching frequency is observed in the spectrogram. Above the spectrogram is a timeline of when the vehicle is underway, and when the vehicle has stopped for a GPS fix. The perceived tone is constant when the vehicle is stationary for a GPS fix, and changes frequency as the vehicle moves closer and further from the array. (b) The measured and expected perceived tone of the switching frequency from the stationary array are compared. . . . . . . . . . . . . . . 109 
4-9 (a) Sidebands centered on the PWM switching frequency of the thruster motor are observed in the spectrogram. Above is a timeline of when the thruster is on (the vehicle is underway) and the thruster is off (the vehicle is at the surface for a GPS fix). Broadband noise interference from boats passing by the UUV is also annotated. (b) Propeller rotation of the UUV is measured over time from the sidebands and compared to the true RPM of the vehicle. . . . . . . . . . . . . . . . . . . . . . . . .

4-10 (a) The true vehicle position, taken from the IMU-based navigation solution of the robot from the Charles River field test, is plotted over time. The coordinate system is with respect to the stationary, dock-mounted array. For comparison, the predicted vehicle position from fusing passive acoustic measurements with a UKF is plotted in $(\mathrm{b}) \ldots \ldots \ldots \ldots$

4-11 The UKF-predicted and true state variables of the UUV from the field test are plotted individually: (a) range, (b) range rate, (c) bearing, and (d) bearing

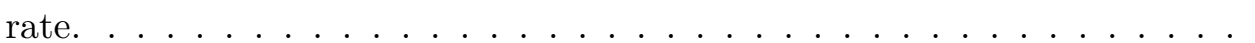

4-12 Error, defined as the mean absolute difference, for the predicted UKF state variables are presented. The columns represent the acoustic measurements used in the UKF. The first column is the baseline case for passive UUV tracking with bearing-Doppler measurements. As the number of available measurements increases, the error in the UKF-predicted state variables decreases. $\ldots \ldots \ldots \ldots \ldots$

4-13 The resulting average error of the UKF prediction for range is plotted for different values of the RPM-speed mapping $(\alpha)$. The dynamic model prediction for the Sandshark UUV $\alpha^{*}$, which is used in the UKF analysis, aligns with the minimum state variable error. . . . . . . . . . . . . . .

5-1 Ray tracing results are presented for extreme shallow water of $10 \mathrm{~m}$ depth, where the UUV is at $1 \mathrm{~m}$ depth: (a) sound speed profile, (b) eigenray paths, (c) incoherent and (e) coherent signal excess for thruster noise, and (d) incoherent and (f) coherent signal excess for fin noise . . . . . . . . . . . 128 
5-2 Ray tracing results are shown for shallow water in the winter of $100 \mathrm{~m}$ depth, where the UUV is at $10 \mathrm{~m}$ depth: (a) sound speed profile, (b) eigenray paths, (c) incoherent and (e) coherent signal excess for thruster noise, and (d) incoherent and $(\mathrm{f})$ coherent signal excess for fin noise . . . . . . . . . .

5-3 Ray tracing results are shown for shallow water in the summer of $100 \mathrm{~m}$ depth, where the UUV is at $10 \mathrm{~m}$ depth: (a) sound speed profile, (b) eigenray paths, (c) incoherent and (e) coherent signal excess for thruster noise, and (d) incoherent and (f) coherent signal excess for fin noise . . . . . . . . .

A-1 Comparison of applying (a) conventional beamforming and (c) MPDR beamforming to the broadband noise from the Sandshark UUV. The respective DOA plots are in $(\mathrm{b})$ and $(\mathrm{d}) \ldots \ldots \ldots \ldots$

A-2 Comparison of applying (a) conventional beamforming and (c) MPDR beamforming to the motor noise from the Sandshark UUV. The respective DOA plots are in $(\mathrm{b})$ and $(\mathrm{d}) \ldots \ldots \ldots \ldots$

B-1 Acoustic data was collected on the radiated noise of the Sandshark UUV in a pool at a high and low speed to estimate the robot's acoustic signature. (a) Power spectral density of the Sandshark UUV at $400 \mathrm{rpm}$. (b) Source level of the UUV above the ambient noise. (c) Power spectral density of the Sandshark UUV at $1000 \mathrm{rpm}$. (d) Source level of the UUV at $1000 \mathrm{rpm}$ above the ambient noise. . . . . . . . . . . . . . . . . . . .

B-2 Acoustic data was collected on the radiated noise of the Sandshark UUV fins in a pool (a) Power spectral density of the fins rotating. (b) Source level of the UUV fins above the ambient noise. . . . . . . . . . . . . .

B-3 Acoustic data was collected on the radiated noise of the Riptide UUV in a pool. (a) Power spectral density of the robot with a desired speed of $1 \mathrm{~m} / \mathrm{s}$ (b) Source level of the UUV above the ambient noise at a desired speed of $1 \mathrm{~m} / \mathrm{s}$. (c) Power spectral density of the robot with a desired speed of $5 \mathrm{~m} / \mathrm{s}$ (d) Source level of the UUV above the ambient noise at a desired speed of

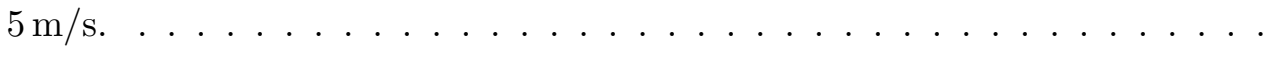

B-4 Comparison of applying the DEMON algorithm to the Sandshark UUV pool test data for (a) broadband noise and (b) high-frequency motor noise . . . 
B-5 Comparison of applying the DEMON algorithm to the Riptide UUV pool test data for (a) broadband noise from $1 \mathrm{kHz}-17 \mathrm{kHz}$, (b) broadband noise from $10 \mathrm{kHz}-17 \mathrm{kHz}$, and (c) high-frequency motor noise. . . . . . . . . . . 140

B-6 Comparison of applying the DEMON algorithm to the Riptide Charles River test for (a) broadband noise from $1 \mathrm{kHz}-17 \mathrm{kHz}$, (b) broadband noise from $10 \mathrm{kHz}-17 \mathrm{kHz}$, and (c) high-frequency motor noise . . . . . . . . . . .

B-7 Comparison of applying the DEMON algorithm to the Sandshark Charles River test for (a) broadband noise from $10 \mathrm{kHz}-17.45 \mathrm{kHz}$ and (b) highfrequency motor noise . . . . . . . . . . . . . . . . . . . 142

C-1 Schematic shows the passive sonar measurements for a UUV approaching an array with constant bearing. The same measurements are produced for (a) and (b) which have different ranges. . . . . . . . . . . . . . . . . . . . . 144

C-2 Schematic shows the passive sonar measurements for a UUV moving around an array at a constant range. A unique solution for range is derived for these measurements. . . . . . . . . . . . . . . . . . . . . 145

C-3 Schematic shows the passive sonar measurements for a UUV moving with a nonzero bearing rate and range rate. A unique solution for range is derived for these measurements. . . . . . . . . . . . . . . . . . . . . 145 


\section{List of Tables}

2.1 List of UUVs that use brushless DC motors for propulsion and are powered by lithium-ion batteries. This combination is common among UUVs of different size, weight, and depth rating $[4] \ldots \ldots \ldots \ldots \ldots$ 


\section{Chapter 1}

\section{Introduction}

Unmanned underwater vehicles have the potential to expand capabilities beyond human limitations, cut costs, reduce human error, and keep humans out of danger in naval operations. For example, one of the first applications of marine robotics in undersea warfare was to handle underwater explosive ordnance. To meet this challenge, the U.S. Navy developed the "Cable-controlled Undersea Recovery vehicle" (CURV) in the 1960s to recover ordnance as deep as $600 \mathrm{~m}$. The CURV became famous in 1966 by recovering a hydrogen bomb from the bottom of the Mediterranean Sea [5].

Cable-controlled vehicles, however, are limited by their tether system: the tether constrains the range of the vehicle, the tether can be damaged during deployment and recovery, and the tether requires a complex management system. The solution to these problems is the untethered, self-propelled unmanned underwater vehicle (UUV). This type of vehicle became an enabling technology for wide-area mine-hunting surveys conducted by the U.S. Navy. Prior to the development of UUVs, shallow-water mine countermeasures (MCM) were carried out by marine mammals and expertly trained divers. In order to reduce risk to personnel, cost, and time of the mission, the man-portable REMUS-100 UUV (remote environmental monitoring units) was developed by the Woods Hole Oceanographic Institution (WHOI) with support from the Office of Naval Research (ONR) to locate and identify mines in shallow water environments [6]. During Operation Iraqi Freedom in 2003, the REMUS-100 UUV successfully performed mine search and clearance in the port city Umm Qasr to allow safe delivery of humanitarian aid [7]. The REMUS-100 UUV technology officially transitioned to the U.S. Navy as the Mk-18 Mod 1 Swordfish program. Then, 
to expand the endurance and improve the sensor performance of the REMUS-100 (7.5 in diameter, $37 \mathrm{~kg}$ weight) for MCM, WHOI created a larger class of UUVs: the REMUS-600 (12.75 in diameter, $240 \mathrm{~kg}$ weight) [8,9]. This new design supported the small synthetic aperture minehunter (SSAM), that provides higher resolution and larger swath width than single beam sidescan sonar. The REMUS-600 also transitioned into the U.S. Navy fleet as the Mk-18 Mod 2 Kingfish.

Inspired by the potential of autonomous undersea systems to meet modern day national security challenges, the U.S. Navy mapped out a vision for future technologies, capabilities, and missions in the The Navy Unmanned Undersea Vehicle (UUV) Master Plan of 2004 [10]. UUVs would be capable of anti-submarine warfare (ASW), collecting intelligence, gathering oceanographic data, acting as a communication node, delivering payloads, and smart mining. For the anti-submarine warfare application, these autonomous platforms could monitor ports and choke points to detect and track adversary submarines. UUVs could also navigate in denied areas or shallow-waters to collect intelligence, and to extend the reach of conventional navy platforms of ships and submarines. Another role for a UUV, as outlined in the "Master Plan," is to act as a submarine decoy or communication jammer [10].

\subsection{Commercial and Research Applications for UUVs}

Because of advances in autonomy, sensing, and navigation, present-day UUVs are capable of completing complex missions. In the search for the missing Malaysia Airlines Flight 370 (MH370), Phoenix International Holdings, Inc. deployed a Bluefin-21 UUV to image the seafloor with sidescan sonar. Although the airplane was not found, two 19th century shipwrecks were discovered [11]. The robot mapped a remote area of the Indian ocean as wide as $860 \mathrm{~km}^{2}$, and in some parts, as deep as $5000 \mathrm{~m}$ [12].

UUVs have also been deployed to explore extreme deep-sea environments like submarine volcanoes [13] and under-ice in the arctic to characterize climate change effects [14]. With advanced autonomy, UUVs can strategically and adaptively sample oceanographic data (such as temperature and conductivity). The data can then be assimilated into ocean models for predicting acoustic propagation [15].

UUVs also have several advantages for observing marine life in situ. Robots are min- 
imally invasive, so the marine life won't attract or avoid. The platforms can be equipped with oceanographic sensors, acoustic sensors, depth sensors, and cameras. Therefore, the behavior of wildlife can be correlated to the environment. For example, researchers at WHOI modified a REMUS-100 to autonomously track sharks that were tagged with an acoustic transponder [16]. By following and imaging the sharks in close proximity, researchers can gain information about the predator's behavior [16]. This approach has also been demonstrated on researching turtle behavior. WHOI developed a REMUS-100 that can follow and image leatherback turtles, where the vehicle is equipped with high-definition cameras and oceanographic sensors (i.e. temperature, salinity, turbidity) [17]. Recently, the Mesobot was developed by a collaborative team of researchers from WHOI, Monterey Bay Aquarium Research Institute (MBARI), and others to study the "ocean twilight zone" [18]. Although this midwater realm is poorly understood, the "ocean twilight zone" - spanning from 200-1000 m depth - hosts the largest fish stock in the ocean and the Earth's largest migration [19]. Researching this ecosystem can help answer questions on climate change and inform policies on human exploitation (i.e. the fishing industry) [19]. The Mesobot is designed for exploring this realm without disturbing the marine life: the robot is capable of tracking zooplankton, fish, and other marine life with a stereo camera [18]. In addition, the platform can sample biogeochemical and environmental DNA [18].

\subsection{The Role of UUVs in the Modern Navy}

Because of UUVs' advanced capabilities, these autonomous platforms are playing an integral part of the Navy's new strategy to move towards Distributed Maritime Operations (DMO) and away from individual, large, and expensive surface ships. The motivation for this approach is in response to enhanced maritime anti-access, area-denial capabilities of other countries [20]. Furthermore, the readiness level of autonomous systems today makes this vision possible and the affordability of unmanned systems makes the strategy cost-effective.

In the introduction to the March 2021 Unmanned Campaign Framework of the U.S. Navy, which is in support of the DMO fleet architecture, the Chief of Naval Operations M. M. Gilday writes:

Unmanned Systems (UxS) have and will continue to play a key part in future Distributed Maritime Operations (DMO), and there is a clear need to field af- 
fordable, lethal, scalable, and connected capabilities. That is why the Navy is expanding and developing a range of unmanned aerial vehicles (UAV), unmanned undersea vehicles (UUV), and unmanned surface vessels (USV) that will play key roles as we shift our focus toward smaller platforms that operate in a more dispersed manner. A hybrid fleet will be necessary for the Navy to meet emerging security concerns. [21]

The purpose of the campaign is to align requirements, acquisition policies, and resources to deliver unmanned systems faster and more effectively. The Navy's 30-year shipbuilding plan for fiscal year 2022, published in June 2021, reflects this vision for a hybrid mannedunmanned fleet: the research and development funding for large unmanned vehicles alone is $\$ 434.1$ million [20] and the inventory of UUVs in the fleet would increase from several prototypes to between 18 and 51 vessels [22]. The portfolio of UUVs includes a range of sizes from the XLUUV Orca (the first five were built in FY2019 by Boeing), which is the size of a subway car, to the Mk-18 Mod 1 Swordfish, which is one-man portable. The expectation for the entire portfolio of unmanned systems is that they continue to extend the reach of the Navy to areas of high-risk, perform missions autonomously with limited human operator communication, and to deploy payloads.

\subsection{The Counter-UUV Problem}

As the United States evolves its own organic AUV (autonomous underwater vehicle) capability, competitors will also be developing their AUV capabilities and cooperating them in close proximity to our own forces. Undersea forces need to develop and deliver the methods, techniques, and tactics to counter AUV detection, survey, and if necessary attack adversary AUVs. [23]

Ray Maybus

75th United States Secretary of the Navy

Autonomous Undersea Vehicle Requirements for 2025

As an essential technology in the modern day navy, UUVs are under development and in operation across the globe for military applications. According to the Jane's report on 
Unmanned Maritime Vehicles from 2015-2016, 21 countries in addition to the United States possess UUVs for military use: Australia, Canada, China, Denmark, France, Germany, Iceland, India, Israel, Italy, Japan, South Korea, Norway, Portugal, Russia, Singapore, South Africa, Sweden, Turkey, Ukraine, and the United Kingdom [4]. In addition to being integrated into major navies, UUVs are an ideal platform for asymmetric warfare. UUVs can be assets to less established navies and non-state actors because they are covert, easy to deploy, low-cost, and low-risk to personnel. While these platforms were once primarily used for mine countermeasures, UUVs of today are capable of being armed and performing offensive missions. As battery, sensing, autonomy, and navigation technology continues to advance, and more vehicles become commercially available, UUVs pose a new threat to ports and harbors, offshore infrastructure, undersea network cables, and navy assets. To counteract this threat, it is necessary to invest in technology to detect, track, and ultimately stop UUVs from causing harm.

For example, DARPA proposed the research program, "Open Ocean Counter Unmanned Underwater Vehicle Study" in 2016 to explore techniques for exploiting vulnerabilities in adversarial UUVs, specifically in open ocean environments [24]. In this program, there were two focus areas. The first focus area was to develop new methods for long-range, accurate detection and classification, and the capability to simultaneously track many vehicles. The second focus area was to find novel techniques for preventing a UUV from carrying out its mission.

In order to prevent armed UUVs from operating near ports and harbors, the Navy (specifically the Strategic Systems Program Nuclear Weapons Security program) published a call for a small business innovation research (SBIR) proposal on UUV detection methods in 2015 [25]. The request required the proposed technology could detect at ranges up to $1000 \mathrm{~m}$ and integrate into existing sonar-based harbor protection systems. In 2017, the Stiletto Maritime Demonstration Program by the Assistant Secretary of Defense for Research and Engineering, Rapid Reaction Technology Office solicited new acoustic methods to detect, classify, and track UUVs in the shallow-waters of ports and harbors [26]. Applying existing technology for detecting combat swimmers and scuba divers near Navy assets and waterside facilities falls short of detecting UUVs in a timely response window. Because existing defense systems are challenged by detecting, tracking, and stopping UUVs from acting on their malicious intent, countering UUVs is a new national security priority. 


\subsection{Summary of Contributions}

In this thesis work, I sought answers to the following questions which were inspired by the counter-UUV problem: What are the sources of machinery noise inside the vehicle, specifically related to the electric motors? What is the performance of the detection of envelope modulation on noise (DEMON) algorithm on UUV cavitation noise? What sources of acoustic noise in UUVs can be exploited for passive detection, classification, localization, and tracking (DCLT)?

I investigated these outstanding questions in the field of radiated acoustic noise in UUVs, which led to the following major contributions:

- Identified common and dominant sources of high-frequency noise in the UUV propulsion system as tones at harmonics of the motor switching frequency.

- Derived a propeller rotation rate estimation method from the unique high-frequency acoustic signature from the motor.

- Collected radiated acoustic noise of UUVs in field tests from a stationary array in the presence of boat traffic in a shallow-water environment.

- Demonstrated the motor noise method is superior to the DEMON algorithm for estimating propeller rotation rate, in shallow-water environments with boat traffic.

- Demonstrated passive acoustic detection, localization, and tracking of a UUV from motor noise with energy thresholding and ROC curve analysis, conventional and adaptive beamforming, measuring the Doppler effect, and applying an Unscented Kalman Filter to the acoustic measurements.

- Demonstrated that Bearing-Doppler-RPM measurements outperformed traditional bearing-Doppler target motion analysis: the tracking solution accuracy of bearing, bearing rate, range, and range rate improved by a factor of $2.2,15.8,3.1$, and 6.2 respectively.

While this thesis is motivated by the perspective of the counter-UUV problem, the contributions of this thesis work are relevant to other problems in the field of radiated acoustic noise of UUVs. Understanding sources of acoustic noise in UUVs can inform the design of quieter propulsion systems. A quiet acoustic signature is necessary for minimally 
invasive observation of wildlife and for avoiding interference with onboard acoustic sensors. Another application of this work is UUV navigation and localization. Onboard the vehicle, the navigation system could use the vibro-acoustic signals from the motor as a low-cost method to measure propeller rotation rate, instead of relying on a costly optical encoder [27]. Lastly, motor noise could be used for UUV localization and tracking generally, as an alternative or supplemental method to acoustic positioning systems.

\subsection{Thesis Organization}

The structure of this thesis begins with an introduction to aspects of UUV design in Chapter 2 that are relevant to the generation of acoustic noise. Also in Chapter 2, I provide background on the key technical concepts of radiated noise in vessels and electric motors. In order to characterize vehicle noise, I built and deployed a reconfigurable, portable acoustic array. I also wrote a library of acoustic signal processing algorithms in Python and MATLAB for analyzing the array data. The details of the array design and signal processing algorithms are in this chapter. Lastly, I include descriptions of the experimental set-ups for the UUV acoustic signature pool and tank tests.

In Chapter 3, I present the acoustic remote sensing method for high-precision propeller rotation and speed estimation of unmanned underwater vehicles. Using this method, in Chapter 4, I demonstrate passive acoustic detection and tracking of an unmanned underwater vehicle from high-frequency motor noise. In Chapter 5, I discuss the effects of the ocean environment that should be taken into consideration for integrating this method into existing passive sonar systems. I also outline future directions to take this work. 


\section{Chapter 2}

\section{Background}

\subsection{Unmanned Underwater Vehicles}

Understanding how unmanned underwater vehicles (UUVs) are built provides insight into the origins of acoustic noise that can be exploited for passive acoustic detection, localization, and tracking. Furthermore, in this section, we provide background information on UUV technologies that are relevant to the vehicles deployed in the field experiments of this thesis. The main subsystems inside the body of a UUV that we will discuss are navigation, control, and propulsion. The navigation subsystem is important to this work because the passive acoustic tracking solution of the UUV position is compared the vehicle's on-board navigation solution. Therefore, the accuracy of the navigation solution should be considered in reporting our results. Motors are utilized in the control and propulsion subsystems of the UUV. These subsystems are described in detail since motors are major contributors to the overall acoustic noise of the vehicle. Lastly, we describe the vehicles which were specifically used in this research.

\subsubsection{Navigation}

Accurate UUV navigation is important for safe recovery and operation of the vehicle, in addition to the utility of the data collected. Because GPS measurements are unavailable underwater, the vehicle can rely on acoustic beacons with a known position as an external reference, dead reckoning, and external environmental information in order to navigate 
underwater. In some cases, the UUV can also periodically resurface for a GPS measurement to reduce navigation error. However, periodic resurfacing costs mission time and energy consumption, particularly for deep-water deployments. For military operations, resurfacing also risks the covertness of the UUV mission.

One approach for accurate underwater navigation is to rely on acoustic beacons or modems as an external reference. The two main categories for this approach are longbaseline or ship-tracked. The long-baseline (LBL) acoustic positioning system involves a network of sea-floor mounted transponder nodes. The challenges of implementing an LBL system include increasing complexity to deploy in deep waters, sensitivity to multi-path, and limited range of a few $\mathrm{km}^{2}$ [28]. Ship-tracked methods- short-baseline (SBL) and ultashortbaseline (USBL) - rely on a surface vehicle to assist the transponder-equipped UUV to navigate. More specifically, the position of the UUV is calculated by measuring range and bearing relative to the surface reference (i.e. a ship) with a known GPS position. Although SBL and USBL require simpler installation and lower maintenance than a LBL system, a challenge of these ship-based systems is accounting for the surface vessel's movements. The advantages and disadvantages of acoustic positioning systems are discussed in detail in Thomson et al. [29] and Vickery et al [30].

When no acoustic positioning system is available, the vehicle relies on inertial navigation or dead reckoning. This is the process where the vehicle estimates its position based on its orientation (from a compass) and integrating measurements from velocity (from a Doppler velocity $\log$ ) or acceleration (from accelerometers and gyroscopes). Because these sensors are subject to noise, navigation error accumulates with distance travelled. In general, vehicles utilize an attitude and heading reference system (AHRS) that fuses measurements from an inertial measurement unit (IMU) and a heading sensor to estimate the vehicle's orientation in the world frame. An IMU is a 3-axis gyroscope, 3-axis accelerometer, and in some cases, 3-axis magnetometer. The resulting pitch, roll, and yaw from the AHRS are inputs to the inertial navigation system (INS), in addition to other sensor inputs (GPS, LBL, USBL, depth sensor, etc.) that ultimately estimates the vehicle position. For UUV operations near the seabed, a Doppler velocity log (DVL) can aid the INS for a more accurate position estimate of the vehicle. The DVL device usually includes four acoustic transceivers that are mounted on the vehicle to be downward facing towards the seafloor bottom. The transceivers send out acoustic pulses to the sea floor, where the pulses are 
reflected off the bottom (establishing bottom lock), and the Doppler shift of the pulses is measured to estimate vehicle speed. The allowable distance between the DVL and sea floor is frequency dependent: low-frequency $(150 \mathrm{kHz})$ requires a minimum distance of $500 \mathrm{~m}$ and high-frequency $(1200 \mathrm{kHz})$ requires a distance of $30 \mathrm{~m} \mathrm{[28].}$

The accuracy of the navigation solution correlates to cost, power, and size constraints of the vehicle. For example, a low-cost UUV (\$4100) with a compass, flow meter, and attitude sensor has a drift rate of $10 \%$, a standard UUV $(\$ 30,000)$ with an INS, DVL and LBL has a drift rate of $1 \%$, and a high-end UUV (\$80,000 dollars) with a FOG-based INS, DVL, and LBL has a drift rate of $0.1 \%$, where drift rate error is percent of the distance travelled [28]. The DVL alone adds significant cost to the vehicle. DVL prices range from $\$ 20,000-\$ 80,000$ [31]. Inside the IMU, the two main types of gyroscopes vary widely with cost and accuracy: ring laser gyroscope drift rate is as low as $0.0001^{\circ} / \mathrm{hr}$ (order of thousands of dollars), and a MEMS IMU is as high as $60^{\circ} / \mathrm{hr}$ (order of hundreds of dollars) [31].

In addition to dead reckoning and acoustic positioning systems, geophysical positioning has been demonstrated for UUV navigation. The vehicle can compare sensor measurements to a priori map of geophysical parameters including bathymetry, magnetic field, or gravitational anomaly [28]. In the situations where no map is available, the UUV can utilize simultaneous localization and mapping (SLAM). In this process, the vehicle simultaneously builds and uses the map for navigation, as new sensor data is collected. SLAM has been demonstrated on UUVs with sonar and camera data [28]. However, underwater cameras are sensitive to turbidity, and consequently have limited range. Although imaging sonar can travel further in water, it is costly and low-resolution [32].

In this work, acoustic noise from low-cost, miniature UUVs were evaluated. Due to cost, power, and space constraints, these vehicles lack a DVL, imaging sonar, or FOG-based INS. The navigation system on these vehicles depends on a MEMS IMU and magnetic compass. Therefore, we expect uncertainty due to drift error in the vehicle's navigation solution. This is important to consider because the navigation solution is used as the true position for evaluating the passive acoustic tracking solution. 


\begin{tabular}{||ccccc||}
\hline \multicolumn{5}{||c|}{ UUVs powered by brushless DC motors with lithium-ion batteries } \\
\hline Name & Manufacturer & Length x Width $[\mathrm{m}]$ & Weight $[\mathrm{kg}]$ & Depth $[\mathrm{m}]$ \\
\hline \hline Sirius & Australian Centre for Field Robotics & $2 \times 1.5$ & 200 & 700 \\
\hline Explorer & International Submarine Engineering & $4.5-6.0 \times 0.69-.740$ & $640-1850$ & $300-6000$ \\
\hline Theseus & International Submarine Engineering & $10.7 \times 1.27$ & 8600 & 2000 \\
\hline REMUS 600 & Kongsberg Maritime Hydroid Inc. & $3.25 \times 0.324$ & 240 & 600 \\
\hline Sentry & Woods Hole Oceanographic Institution & $2.9 \times 2.2$ & 1250 & 6000 \\
\hline Iver2 & OceanServer Technology, Inc. & $1.27 \times 0.147$ & 19 & 100 \\
\hline Iver3 & OceanServer Technology, Inc. & $1.5-2.16 \times 0.147$ & 38.5 & 100 \\
\hline REMUS 100 & Kongsberg Maritime Hydroid Inc. & $1.6 \times 0.19$ & 37 & 100 \\
\hline
\end{tabular}

Table 2.1: List of UUVs that use brushless DC motors for propulsion and are powered by lithium-ion batteries. This combination is common among UUVs of different size, weight, and depth rating [4].

\subsubsection{Control and Propulsion Systems}

The main objectives of the UUV propulsion and control systems are to stay at depth and to adeptly maneuver underwater. In a typical UUV configuration, these objectives are accomplished by a screw-type propeller powered by an electric motor that drives the vehicle forward, and rudder, stern, and potentially, sail planes controlled by actuator motors that enable yaw and pitch motion $[5,33]$. The main propeller of the vehicle is most commonly a screw propeller because it is the most efficient propeller design for marine propulsion [5]. The propeller produces thrust by forcing fluid backwards. The amount of fluid depends on the propeller's pitch angle and its speed of rotation.

The electric motor type that drives a majority of UUV propulsion systems is the brushless DC motor, powered by a lithium ion battery [34]. In fact, Table 2.1 is a list of vehicles that rely on brushless DC motors for propulsion and are powered by lithium ion batteries [4].

This popular motor type is utilized in many robots because it has the following positive attributes: commercially available, high-efficiency, low-maintenance, long-life, low weight, compact size, low-cost, and high torque-to-inertia ratio $[35,36]$.

When driving the propeller by an electric motor, there are three common strategies for preventing water ingress. First, the motor can be housed in a pressure vessel and use rotating shaft seals. However, this strategy results in large friction losses, decreasing the overall efficiency of the motor. Second, the need for seals can be eliminated through magnetic coupling. The challenge of this strategy is an increase in cost and size of the 
thruster. Furthermore, synchronous coupling fails when the vehicle experiences a sudden acceleration. Third, the motor can be housed under-pressure in oil. The oil is chosen such that it is compatible with the motor winding insulation and electronics. By maintaining the oil at a higher pressure than the sea water, oil will leak out instead of the sea water leaking into the housing.

Most commonly, the UUV hull, like a torpedo or submarine, is a stream-lined, tear-drop shaped cylinder that is optimized for hydrodynamics. The rudder is placed at the stern of the vehicle and controls yaw (the heading). Also at the stern are a set of hydroplanes that act like the elevators of an aircraft to control pitch. Using a dynamic model of the vehicle, a heading controller, pitch controller, and depth controller can be implemented to maneuver the vehicle to the desired orientation.

The hydroplanes of the vehicle are controlled by rotary actuators. The main categories of electric motor rotary actuators are stepper motors and servomotors, which have some key differences. Stepper motors rotate in small angle increments, in response to a series of controlled discrete pulses. Once the stepper motor rotates to the precise angle, it halts, without any position feedback. In contrast, servomotors provide more precise control. Servomotors utilize position feedback with a rotary encoder. Servomotors are controlled using pulse width modulation where the varying width corresponds to the desired rotor angle position. Servomotors can also handle variable load systems. As a consequence to better precision with closed-loop feedback, servomotors are more complex and expensive than stepper motors.

\subsubsection{Low-cost, Micro-UUV Design}

The BAE Systems Riptide $\mu$ UUV [37] and the General Dynamics Mission Systems Bluefin Sandshark UUV [38] were used in this research study. These robots belong to the smallest class of vehicles called "micro-UUVs" which have a diameter of six or less inches. These vehicles are appealing because they are one-man portable: they can be easily deployed from boats, ships, and submarines. As technology in autonomy and communication improves, a collaborative and autonomous swarm of micro-UUVs could potentially outperform a single vehicle with a complex sensor package for collecting oceanographic data over large areas. For example, underwater plumes, which could include harmful algae blooms, oil spill plumes, or 
hydrothermal vent fluid, rapidly evolve in space and time. In order to capture the plume's time-varying, spatial content, a network of vehicles can be deployed to track the plume boundary [39]. A single vehicle, in contrast, would struggle to characterize a fast-moving, large plume. Another use case for small vehicle swarms is to efficiently search the ocean for targets, such as black boxes of submerged planes. While one vehicle would need to follow a time-consuming search pattern, a group of vehicles could work together to cover a wide-area. Lastly, deploying multiple robots provides redundancy to any mission- where if one vehicle malfunctions, another vehicle can take over its role, and the mission can still proceed.

A significant challenge of low-cost, miniature UUVs, however, is navigation. The vehicle is constrained in size, weight, power, and cost. Therefore, this class of vehicles is configured with a low-cost MEMS inertial measurement unit and magnetic compass, rather than a Doppler velocity log and fiber optic gyroscope inertial navigation system. An approximation of the drift error rate for the Sandshark navigation solution (low-cost IMU-based navigation system) for position is $1 \mathrm{~km} / \mathrm{hr}$ [40].

\subsubsection{Sandshark UUV}

The General Dynamics Mission Systems Bluefin Sandshark UUV [38], pictured in Figure 2-1 was used to demonstrate passive acoustic detection and tracking from high-frequency motor noise. The robot is designed for shallow-water deployments up to $200 \mathrm{~m}$ depth with a top vehicle speed of $2.5 \mathrm{~m} / \mathrm{s}$ [38]. Its compact hull design has a diameter of $0.12 \mathrm{~m}$ (4.875 in), which matches the standard sonobuoy launcher diameter. The inside of the vehicle is configured into two sections: a standard tail section provided by the manufacturer and a custom payload section. The tailcone section houses the thruster, actuators, altimeter, and battery. The vehicle propeller is powered by a brushless DC motor through magnetic coupling. Three fins, which are steered by servomotors, control the vehicle's roll, pitch, and heading. For navigation, the vehicle utilizes a MEMS IMU with a 3-axis gyroscope, 3-axis magnetometer, and 3-axis accelerometer. The inertial measurements are fused with a direction cosine matrix algorithm to estimate the vehicle's orientation as an inexpensive AHRS [41]. The speed-over-ground is estimated from the propeller RPM. In order to estimate depth, the vehicle relies on a single-beam echo sounder and pressure-temperature 


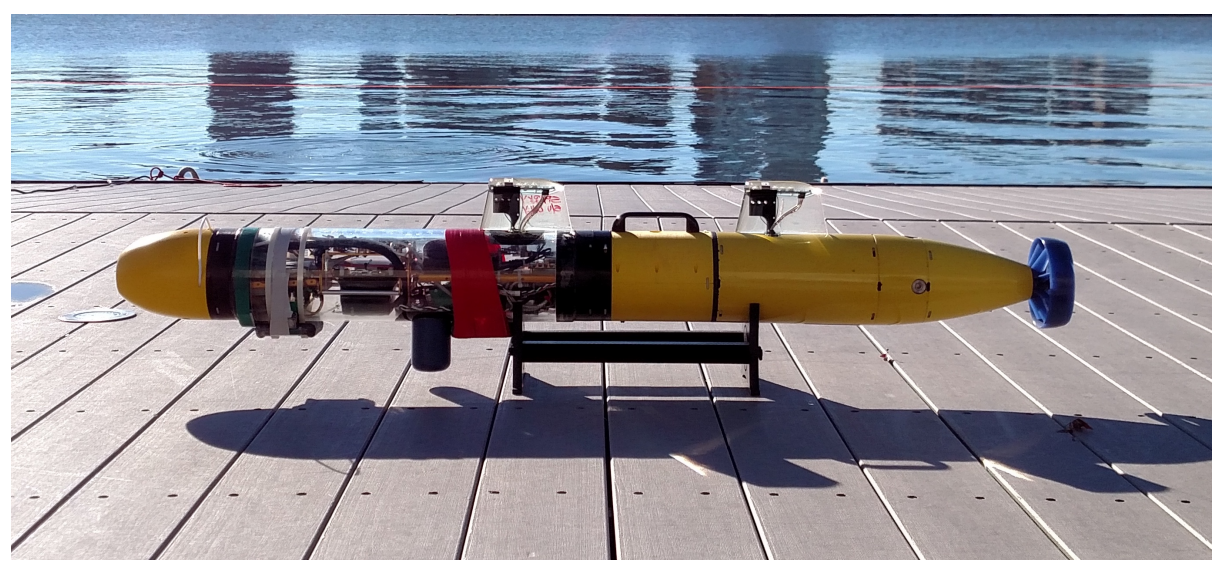

Figure 2-1: The Sandshark UUV is used in field testing in the Charles River of Boston, Massachusetts.

sensor. Inside the mast of the vehicle are receivers for GPS and WiFi for navigation and communication respectively. The robot employs a proportional-integral-derivative controller to achieve a desired depth, pitch, and heading. The autonomous missions and behaviors of the vehicle are programmed with open-source MOOS-IvP autonomy software [42]. The payload computer, which runs the MOOS-IvP framework, sends commands for desired depth, speed, and heading. Therefore, the high-level autonomy is handled by the payload computer, and the main vehicle computer runs the low-level controls and sensors.

\subsubsection{Riptide UUV}

The BAE Systems Riptide UUV (Mk1 version), pictured in Figure 2-2 was selected for researching acoustic noise that radiates from vehicles [37]. This robot is part of the microUUV class of vehicles with a diameter of $0.12 \mathrm{~m}$ (4.875 in). The vehicle is designed to reach a maximum depth of $300 \mathrm{~m}$ and top speed of 10 knots. The MOOS-IvP software architecture is used for the vehicle controls, as well as the autonomy. Because the vehicle software is open-source, it is consequently highly-customizable to the end-user. The navigation sensors include a 9-DOF IMU and magnetometer, altimeter, temperature sensors, and pressure sensors. The vehicle is stabilized by three individually actuated fins. The vertical control fin also houses the GPS and Wifi receivers. For power, the vehicle relies on 144 alkaline AA batteries. 


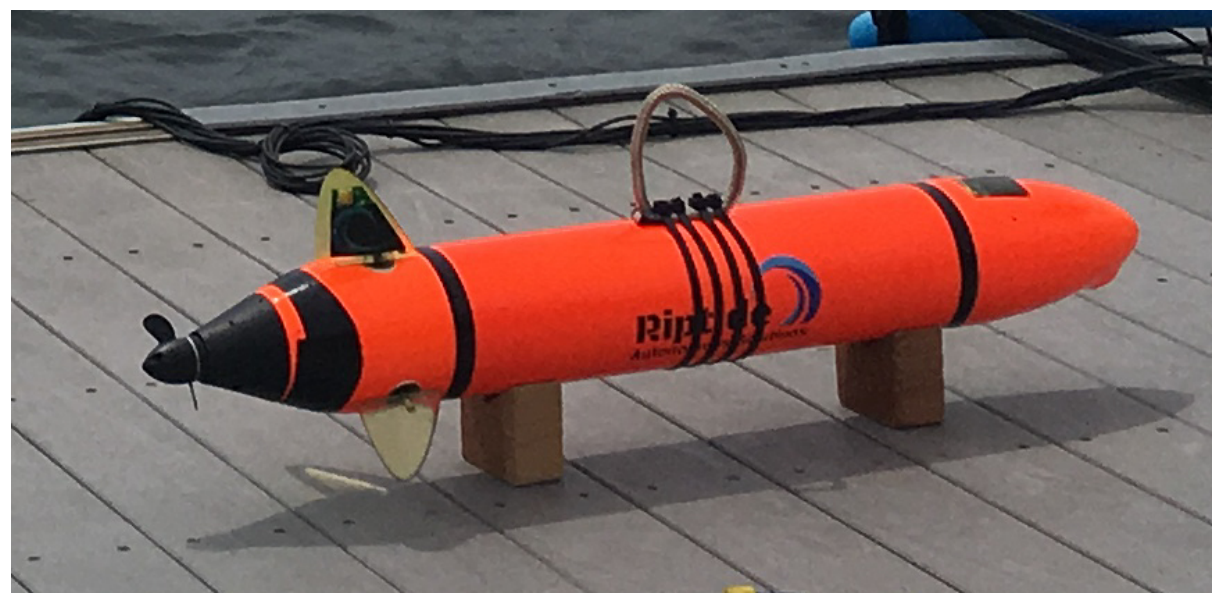

Figure 2-2: The Riptide UUV is tested in field experiments in the Charles River of Boston, Massachusetts.

\subsection{Radiated Acoustic Noise of Vessels}

The first step in effectively tracking UUVs with passive acoustics is to understand the major sources of noise generated by the vehicle. In general, acoustic noise radiates from vessels due to rotating and reciprocating mechanisms such as engines, motors, and gears. The repetitive motion generates vibration, "machinery noise", which can transfer to the surrounding water through the hull as sound that a distant hydrophone can pick up. Some examples of machinery noise include: rotating unbalanced parts (i.e. motor armatures), mechanical friction (i.e. bearings), repetitive discontinuities (i.e. armature slots, gear teeth), cavitation and turbulence (i.e. fluid in pumps, valves, and pipes), and reciprocating parts (i.e. cylinders in engines) [1]. An example of how machinery noise is generated on a dieselengine system is in Figure 2-3, which is adapted from Urick et al..

Another major source of noise originates from propeller cavitation. Broadband cavitation noise contains "propeller beats" where the cavitation noise is amplitude-modulated at the rotation of the propeller, or at the shaft rotation frequency multiplied by the number of propeller blades [1]. Low frequency tones called "blade lines" are also present in propeller noise at harmonics of the shaft rotation frequency multiplied by the number of blades [1]. These features, propeller beats and blade lines, can be used for vessel classification and for estimating the target's speed $[1,43]$. The process for extracting these features from broadband cavitation noise is the detection of envelope modulation on noise (DEMON) algorithm. The process for the DEMON algorithm is as follows: (i) a bandpass filter is applied to the 


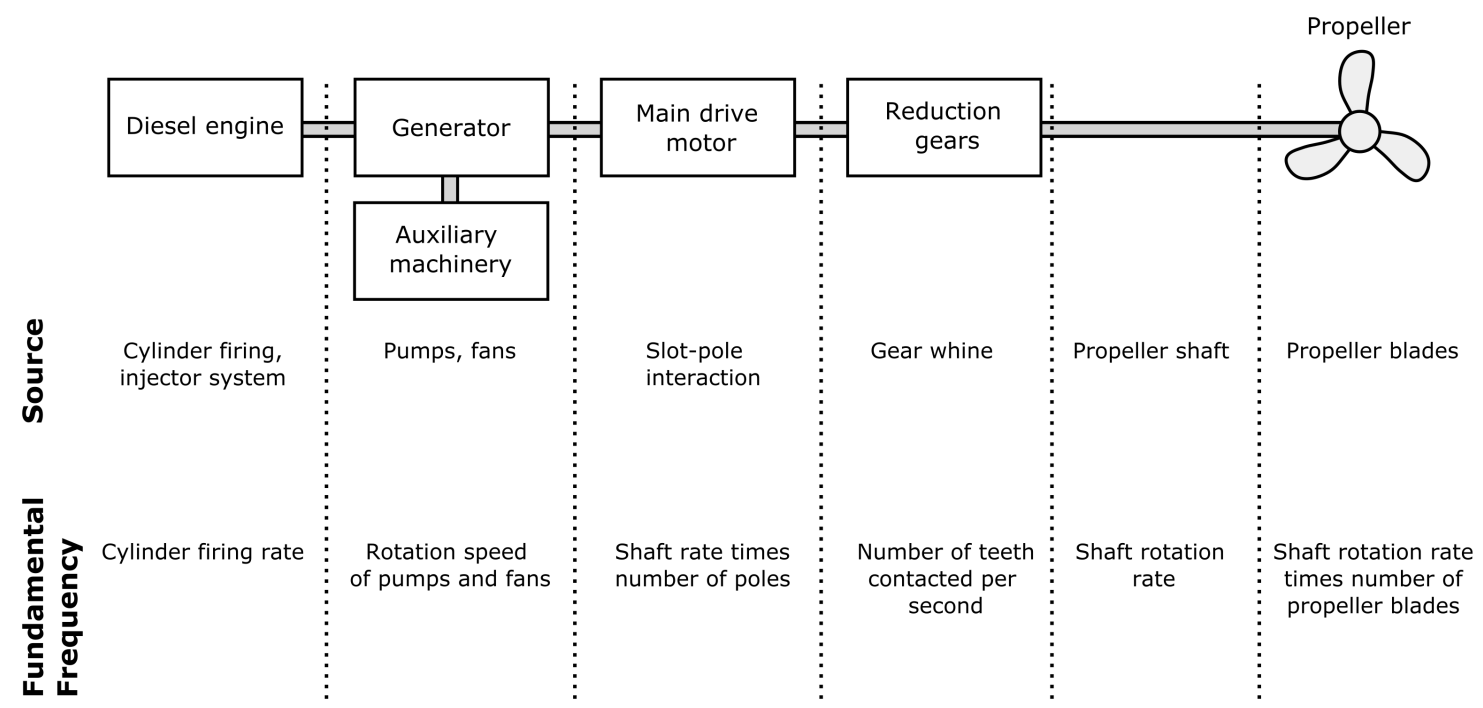

Figure 2-3: Diagram of how machinery noise is generated inside a diesel-engine machine. Adapted from Urick et al. [1]

acoustic data to capture the propeller noise (ii) the amplitude envelope is determined by the magnitude of the Hilbert transform applied to the bandpass filtered signal (iii) the amplitude envelope is downsampled using the root mean square (RMS) method described in Chung et al [44] (iv) Finally, the DEMON spectrum was calculated from the FFT of the amplitude envelope.

This process has been demonstrated successfully on small boats $[45,46]$ and ships [44] for the purpose of small vessel DCLT in ports and harbors. However, using cavitation noise for passive tracking has several limitations. Cavitation noise has a characteristic radiation pattern that is weaker in the fore-and-aft directions, and varies with speed and depth of the target [1]. Therefore, the target could maneuver in an orientation or dive to a certain depth to decrease the SNR of its acoustic signature related to cavitation. To illustrate the effect of speed and depth on the cavitation noise of a hypothetical submarine, Figure 2-4 is a diagrammatic cavitation noise spectrum. The peak noise level occurs at the higher speeds, in lower depths.

A challenge of detecting UUV cavitation noise is that these smaller vessels generate less noise than the cavitation of ships and boats. In the ambient noise of harbors, which includes the sounds of ship traffic, propeller beats and blade lines of the vehicle are challenging to measure. Furthermore, another challenge for DEMON analysis is the selection of the propeller noise passband filter, which can significantly affect the algorithm's performance. 


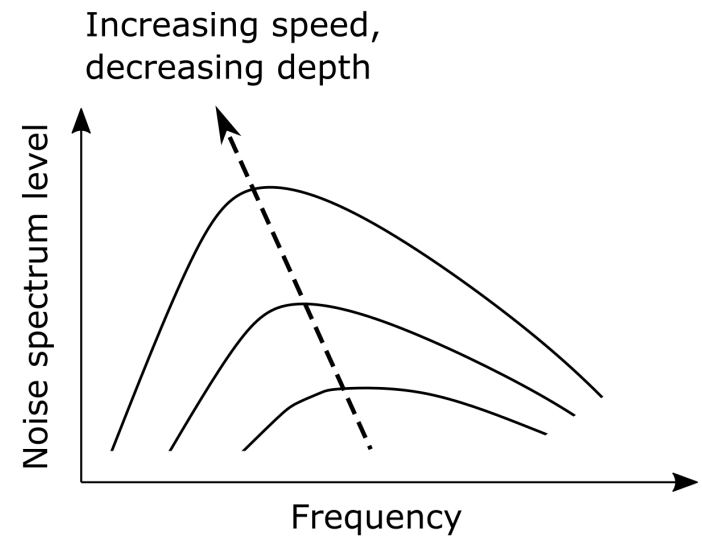

Figure 2-4: Cavitation noise of submarines increases with higher speeds and lower depths. Adapted from Urick et al. [1]

The ideal passband filter varies widely between vessels and is chosen generally based on trial and error [45].

Because of the limitations in using cavitation noise for target motion analysis, we focus on sources of machinery noise, particularly motors, in these platforms. The UUV system has two modules of notable size and power which have the potential to create unwanted machinery noise: motors for stability and a motor for propulsion. We investigate acoustic features of the motors inside UUVs that can be exploited for the purpose of detection, classification, localization, and tracking.

\subsection{Permanent Magnetic AC Synchronous Mo- tors}

The unmanned underwater vehicles (UUVs) that were selected for study rely on permanent magnet AC synchronous (PMAC) motors. The rotational speed of the rotor inside PMAC motors is the same speed as the rotating field of the stator (thus called "synchronous"). These motors are appealing for high-performance applications in robotics, that require high power density, long life-span, electrical efficiency, and high torque-to-inertia ratio. In addition to the Sandshark and Riptide robots that are evaluated in this thesis, PMAC motors, specifically the brushless DC motor, are found in the REMUS-100/600/6000, Sentry, Dorado-class, Iver2, [47]; Riptide [48], and Autosub [49]. In this section, we give a detailed 
overview of how these motors work, as well as how the motor design contributes to acoustic noise in this popular motor type. The vibration and noise originating from the motor produces tones that can be ultimately used for passively tracking the UUV.

The two main types of PMAC motors are distinguishable by their back-EMF: sinusoidal (permanent magnet synchronous motors) and trapezoidal (brushless DC motors). BackEMF stands for the electromotive force from an induced field that opposes the applied voltage that induced it in the first place. This phenomena is defined as Faraday's Law:

$$
\varepsilon=-N \frac{d \Phi_{B}}{d t}
$$

where the direction of the force is determined from Lenz's Law. The $\varepsilon$ is the EMF, and $\Phi_{B}$ is the magnetic flux through the closed loop. The back-EMF is proportional to motor speed.

The mechanical equation that describes a PM motor is:

$$
J \frac{d \omega_{m}}{d t}=T_{e}-T_{\text {load }}-T_{\text {cogg }}
$$

where $T_{e}$ is the electromagnetic torque, $T_{\text {load }}$ is the load torque, and finally, $T_{\text {cogg }}$ is cogging torque [50]. The electromagnetic torque, $T_{e}$, for a 3 -phase PM motor is is:

$$
T_{e}=\frac{e_{a} i_{a}+e_{b} i_{b}+e_{c} i_{c}}{\omega_{m}}
$$

with the back-EMF voltages $e_{a}, e_{b}$, and $e_{c}$, and phase currents $i_{a}, i_{b}$, and $i_{c}$. In order to produce a maximum torque, the phase currents need to be in phase with the back-EMF

voltages. A major source of acoustic noise in PMAC motors is due to the vibration from tangential (cogging torque and ripple torque) and radial electromagnetic forces. Acoustic noise occurs when this vibration aligns with a mechanical resonance in the motor system.

\subsubsection{Torque Ripple}

Ripple torque is defined as the pulsating torque components due to the interaction between the stator current magnetomotive forces and the rotor electromagnetic properties [51]. Torque "ripple" occurs when there is misalignment between the phase currents and backEMF voltages. Therefore, permanent magnet synchronous motors (PMSMs) (the stator 


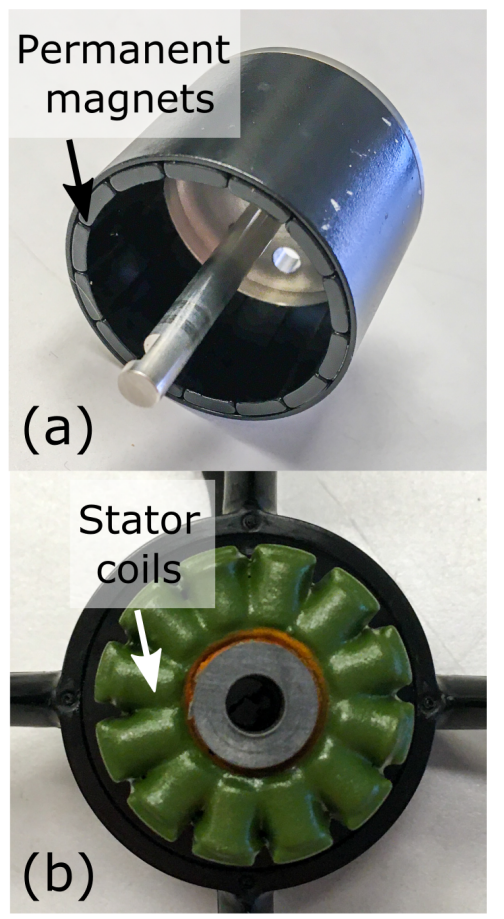

Figure 2-5: Image of the inside of a BLDC motor: (a) permanent magnets on the rotor (b) stator coils.

windings are sinusoidally distributed) use sinusoidal phase currents to drive the motor. Brushless DC motors have concentrated stator windings which result in a trapezoidal backEMF. These motors are consequently electronically commutated with a quasi-square wave called six-step commutation. The inside of a BLDC motor is pictured in Figure 2-5. A diagram of the BLDC motor in Figure 2-6 shows how the the coils are energized sequentially to interact with the permanent magnets on the rotor to ultimately rotate the motor.

Torque ripple is an issue in BLDC motors with quasi-square wave commutation because the phase currents cannot instantaneously rise and fall. To illustrate this, Figure 2-7 is a diagram of one of the phase currents, back-EMF, and resulting torque. In six-step commutation, the torque ripple generated is consequently at the harmonics of $f_{t r}$ :

$$
f_{t r}=6 f_{s}
$$

[52].

Cogging torque, like ripple torque, produces undesired, harmonic torque pulsations. 

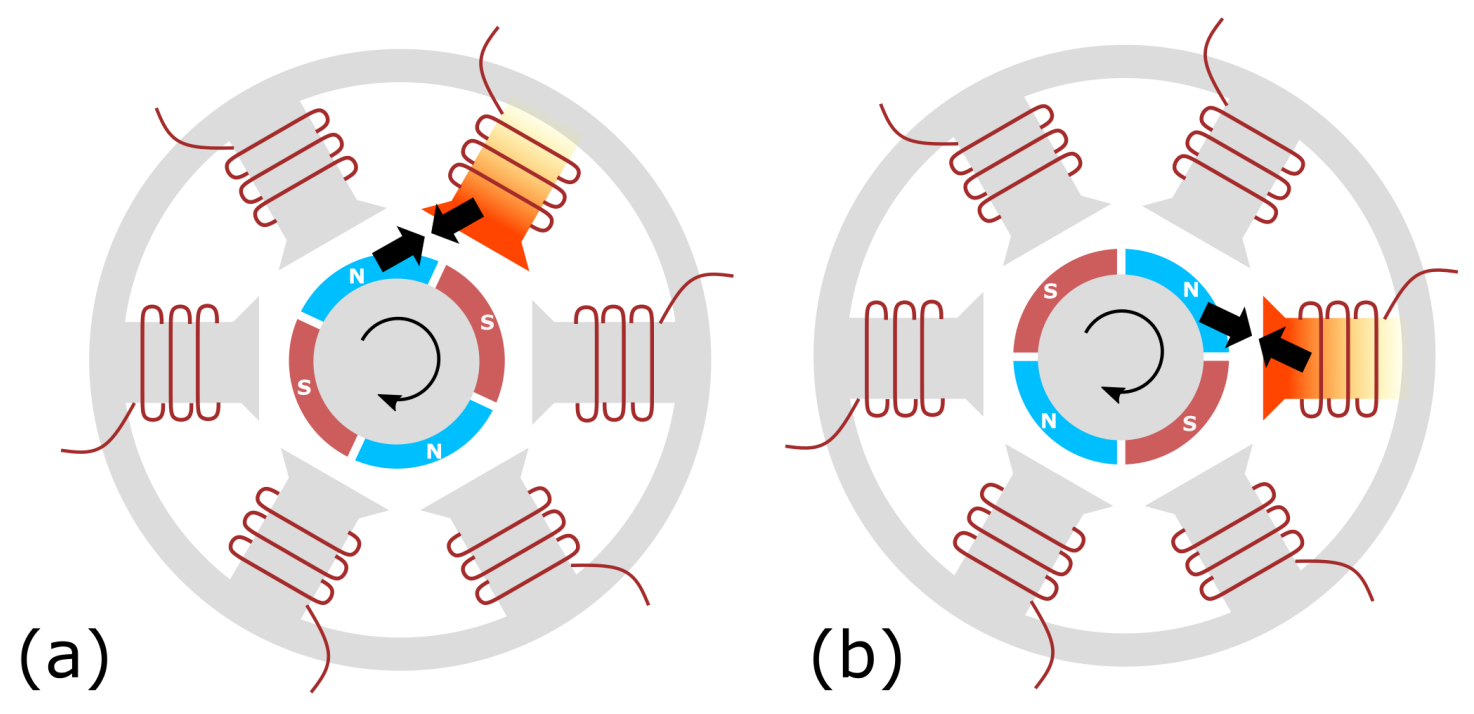

Figure 2-6: Diagram of the inside of a brushless DC motor. The rotor (the part that rotates) has permanent magnets which interact with the energized coils on the stator (stationary part). The rotor rotates sequentially as the coils are energized as shown from positions (a) to (b).

\subsubsection{Cogging Torque}

Cogging torque occurs when the stator is not excited, and is caused by the interaction between the rotor magnetic flux and the stator magnetic reluctance [51]. This phenomena leads to vibration at the harmonics of $f_{\text {cog }}$ :

$$
f_{\text {cog }}=\frac{L C M f_{s}}{p}
$$

or

$$
f_{\text {cog }}=s f_{s}
$$

where $L C M$ is the least common multiple of the number of stator coils and permanent magnet poles, $f_{s}$ is the shaft rotation frequency, $s$ is the number of stator coils, and $p$ is the pole number $[52,53]$. Cogging torque can be reduced by modifying the permanent magnet pole design, such as skewing the poles or adding magnetic bridges [54]. However, these design changes increase the cost and complexity of mass-producing these motors, as discussed in Islam et al [55]. 


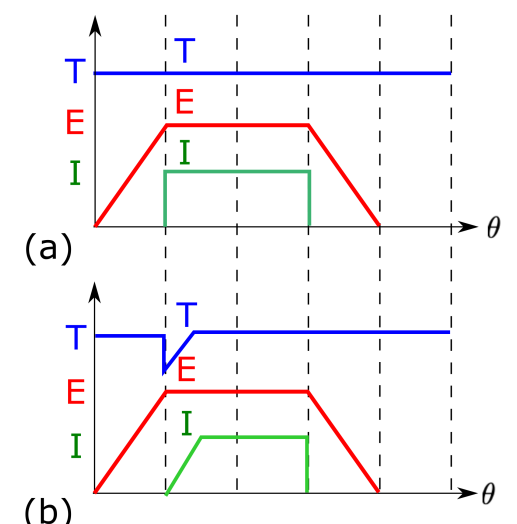

Figure 2-7: The torque output (T), back-EMF (E), and current (I) are plotted for one-phase. (a) Constant torque results from an ideal quasi-square wave. (b) Torque ripple occurs in reality because current can't instantaneously rise and fall. Image modified from $[2]$

\subsubsection{Commutation Methods}

The other main type of PMAC motors, PMSM motors, have a distributed winding and a sinusoidal drive scheme that reduces torque ripple. However, the power density of a BLDC is $15 \%$ higher [36]. The sinusoidal commutation scheme requires higher resolution rotor position sensing (such as an optical encoder), which increases the cost and complexity of the motor.

The three main strategies for driving a BLDC motor (trapezoidal back-EMF) are: sixstep, sinusoidal, and field oriented control (FOC) (or vector control). Six-step or trapezoidal commutation uses low-cost Hall-effect sensors to measure the position of the rotor and follows a simple predetermined sequence from two switching power devices [56]. This commutation sequence is illustrated in Figure 2-8. For an ideal trapezoidal back-EMF inside a BLDC motor, torque is maximized when the current and back-EMF are maximum. While this commutation scheme is easy to implement and optimizes speed and torque output, it produces significant, audible torque ripple.

BLDC motors can also be controlled by sinusoidal commutation because the back-EMF is not perfectly trapezoidal [57]. Using sinusoidal commutation is advantageous for eliminating torque ripple, and is consequently quieter than trapezoidal control. However, because the current phases are overlapping in this commutation scheme, precise rotor position measurements are needed. Sinusoidal control typically utilizes an encoder and Hall-effect sensors 


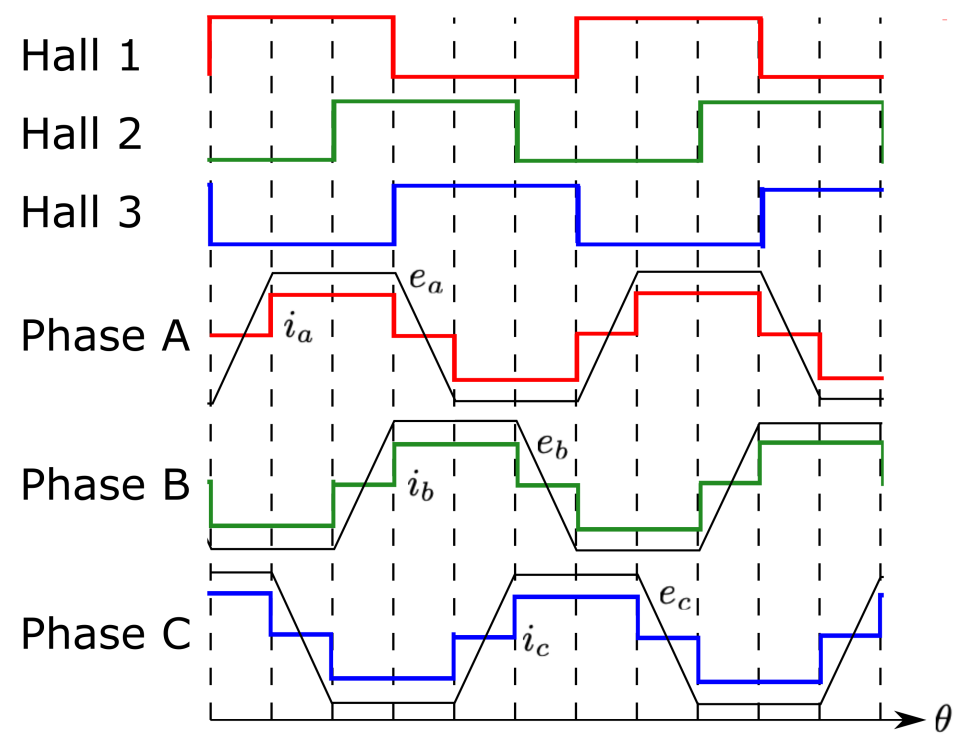

Figure 2-8: Trapezoidal or six-step commutation for a brushless DC motor with Hall sensors is illustrated.

with a look-up table for timing the phase currents [56].

Field oriented control (FOC) or vector control directly controls the current space vectorboth the magnitude and phase of the motor current- and consequently provides instantaneous torque control. The instantaneous torque, $T$, of a motor is determined by

$$
\begin{array}{r}
T=k(\boldsymbol{\phi} \times \boldsymbol{i}) \\
T=k|\boldsymbol{\phi}||\boldsymbol{i}| \sin (\theta)
\end{array}
$$

where $\boldsymbol{i}$ is the current vector, $\phi$ is the magnetic flux vector, and $\theta$ is the space angle between the two vectors [58]. In FOC, the space angle is always 90 degrees, and both the field flux and current can be controlled independently. In PMAC motors, the field flux produced by the permanent magnets on the rotor is inherently independent from the stator winding, torque-producing current. The current can be controlled instantaneously be using a PWM inverter and current regulator [58].

\subsubsection{Sensorless Control Techniques}

Encoders, Hall-effect sensors, and resolvers are utilized in BLDC motors to measure the rotor position for commutation. In BLDC motors, which have a trapezoidal back-EMF, only six 
phase commutation instants occur per electrical cycle, so low-cost Hall-effect sensors are commonly used. In PMSM motors, which have a sinusoidal back-EMF, continuous rotor position information is needed to perform commutation. Thus a high resolution sensor is needed - such as a resolver or shaft encoder.

The advantage of implementing a sensorless BLDC motor is lower cost and potentially, space savings. A sensorless motor relies on only electrical measurements, and does not require position sensors. One strategy of sensorless control is measuring the back-EMF. When the motor is spinning fast enough, about 500-1000 rpm- the back-EMF can be used to estimate motor speed [59]. For example, the back-EMF zero crossing detection method involves measuring the induced voltage on the non-powered phase [59,60]. Acarnley et al. and Gamazo-Real et al. provide a review of sensorless techniques in permanent magnet machines, as well as their trade-offs [60,61].

\subsubsection{Radial Electromagnetic Forces}

Radial electromagnetic forces due to the air-gap flux density in PMAC motors are a function of the slot/pole combination, current harmonics, and back-EMF harmonics. These radial forces lead to deformation in the stator core, causing vibration. The radial force harmonics can be written as:

$$
F_{r m}=\sum_{n} \sum_{l} F_{r m n l} \cos \left[n p \omega_{r} t-\left(n p \pm l N_{s}\right) \theta\right]
$$

were $p$ is the number of poles number, $N_{s}$ is the slot number, $u=2 n+1, k=n(n=0,1,2 \ldots)$ are the harmonics, and $\omega_{r}$ is the rotation frequency of the motor in radians $f_{1}=\omega / 2 \pi[62]$. Therefore, the frequency and mode number of the radial force harmonics are:

$$
\left[n p f_{1},-n p \pm l N_{s}\right]
$$

with $n, l=0,1,2 \ldots[62]$. Thus, when the motor rotates, the excitation frequency $f_{r}$ of the radial force on each stator tooth is:

$$
f_{r}=p f_{s}
$$

where $p$ are the poles of the motor, $f_{s}$ is the shaft rotation frequency $[63,64]$.

The motor speeds- for both BLDC and PMSM- are controlled using pulse-width modulation (PWM) [36,65]. Although PWM is an efficient method for controlling motor speed, 
harmonics of the PWM switching frequency in the phase current produce radial vibration. Several groups have determined, through simulation and experimentation, that vibration from the radial electromagnetic force at the PWM current harmonics produce strong tones in BLDC motors [64], PMSM motors [66,67], and induction motors [68-70]. In Zeze et al., the authors demonstrated experimentally that the vibration mode centered about the PWM carrier frequency is dependent on pole number.

There are several techniques for reducing noise due to the PWM current harmonics, including randomized PWM [71]. As summarized in Lo et al., these techniques, come at an expense, such as efficiency [69].

\subsection{Acoustic Array Design and Signal Processing Methods}

The purpose of array processing is to linearly combine signals from an array of sensors with a certain weighting in order to examine signals arriving at a particular angle. This process enhances the signal from a particular angle through constructive interference and attenuates signals from other directions by destructive interference, thus forming a "beam" and acting as a spatial filter. In this thesis research, beamforming is used to estimate the direction of arrival, or bearing of the UUV by spatially filtering for the vehicle noise.

First we introduce the theory of conventional beamforming on a line array, which was the array geometry used in this thesis work. Because array performance depends on a number of factors, we also discuss how to optimize the array parameters for the target we are interested in tracking. Then we introduce discrete Fourier transform broadband beamforming, which was utilized in this analysis. After discussing the theory behind beamforming, we give an overview of the array that was designed and built for the UUV field experiments. Lastly we discuss how the effect of temporal and spatial aliasing is accounted for in the array processing analysis.

\subsubsection{Conventional Beamforming}

In order to apply conventional beamforming to UUV tracking, we assume that the vehicle is far enough from the array (range $r$ is much greater than the wavelength, $r>>\lambda$ ) such 


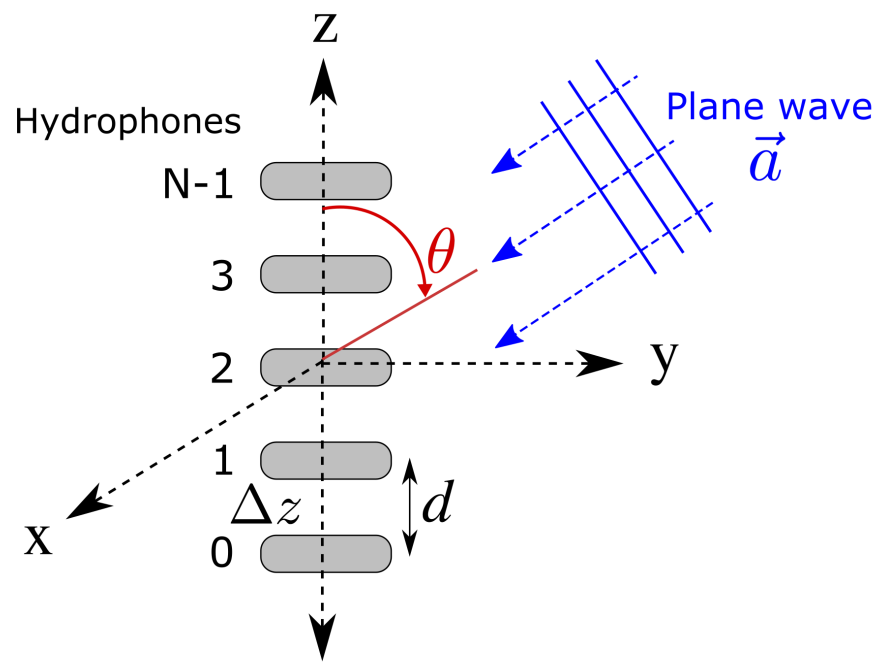

Figure 2-9: Line array diagram

that the acoustic noise radiating from the vehicle can be approximated as a plane wave. In this section, we first derive the frequency-wavenumber response of the array for a unit plane-wave on a uniform line array. In this array set-up, the sensors positioned in a line to sample the signal in space, as pictured in Figure 2-9. The locations of the sensors are equally spaced apart at distance $d$. With the center of the array at the origin of the coordinate system, the position of each element is:

$$
\begin{aligned}
p_{z_{n}} & =\left(n-\frac{N-1}{2}\right) d \\
n & =0,1, \ldots, N-1
\end{aligned}
$$

A vector representing the sensor output of the array is:

$$
\mathbf{f}\left(t, p_{n}\right)=\left[\begin{array}{c}
f\left(t, p_{0}\right) \\
f\left(t, p_{1}\right) \\
\vdots \\
f\left(t, p_{N-1}\right)
\end{array}\right] .
$$

A linear, time-invariant filter with impulse response $h_{n}(\tau)$ is applied to each sensor $n$ output signal, and the filtered sensor outputs are summed to create the array output $y(t)$ :

$$
y(t)=\sum_{n=0}^{N-1} \int_{-\infty}^{\infty} h_{n}(t-\tau) f\left(t, p_{n}\right)
$$


or in vector notation:

$$
y(t)=\int_{-\infty}^{\infty} \mathbf{h}^{T}(t-\tau) \mathbf{f}(t, \mathbf{p})
$$

where

$$
\mathbf{h}(\tau)=\left[\begin{array}{c}
h_{0}(\tau) \\
h_{1}(\tau) \\
\vdots \\
h_{N-1}(\tau)
\end{array}\right]
$$

In order to demonstrate beamforming, we consider the array output to an incoming plane wave from direction a and frequency $\omega$. The signal received at the origin from the incoming plane-wave is defined as $f(t)$. Therefore, the signal received at the array elements is the same signal but with a time-delay, as a function of their position:

$$
\mathbf{f}\left(t, p_{n}\right)=\left[\begin{array}{c}
f\left(t-\tau_{0}\right) \\
f\left(t-\tau_{1}\right) \\
\vdots \\
f\left(t-\tau_{N-1}\right)
\end{array}\right]
$$

The time delay is a function of the speed of sound in the medium $c$, the direction of the incoming wave $\theta$, and the position of the array element $p_{z_{n}}$ :

$$
\tau_{n}=-\frac{1}{c}\left[\cos (\theta) p_{z_{n}}\right]
$$

Considering the received signal $f(t)$ in the frequency domain $F(\omega)$, the $n$th element component of $F(\omega)$ is

$$
F_{n}(\omega)=e^{-j \omega \tau_{n}} F(\omega)
$$

using the time shifting Fourier transform property. The time-delay can be written in terms of the $z$-component of the wavenumber $\mathbf{k}$, for the line array case, as:

$$
\omega \tau_{n}=k_{z} p_{z_{n}}
$$

where:

$$
k_{z}=-\frac{2 \pi}{\lambda} \cos (\theta)
$$




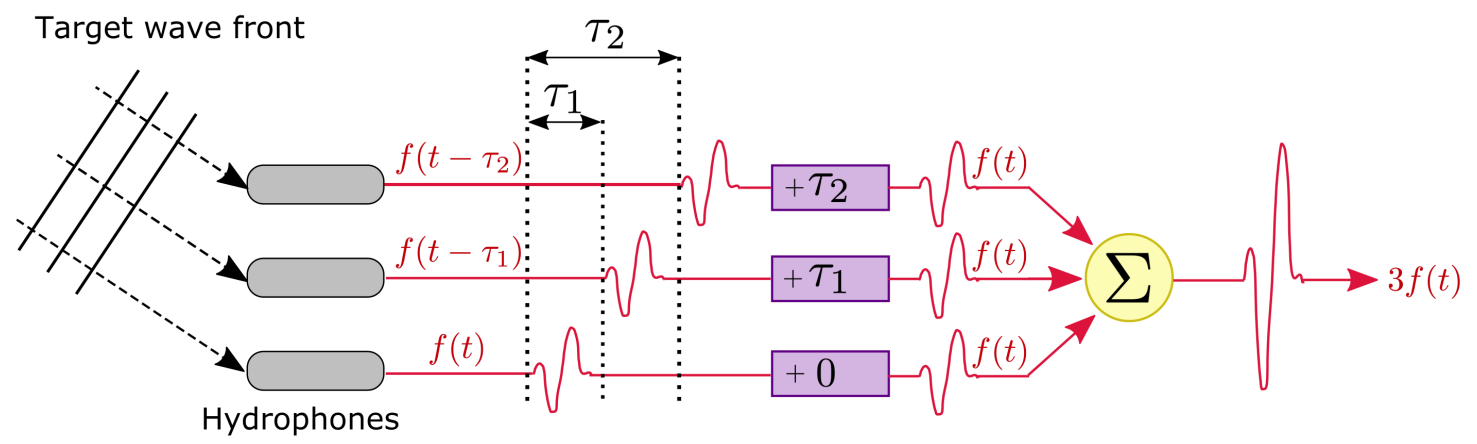

Figure 2-10: Delay-and-sum beamformer for an incoming plane wave $f(t)$

with a constant magnitude equal to:

$$
|\mathbf{k}|=\frac{\omega}{c}=\frac{2 \pi}{\lambda} .
$$

Therefore, the received signal on the array in the frequency domain can be rewritten in vector form as:

$$
\mathbf{F}(\omega)=F(\omega) \mathbf{v}_{k}(\mathbf{k}) .
$$

The array manifold vector $\mathbf{v}_{k}(\mathbf{k})$ accounts for the time-delay at each element, as a function of the element's position. For a line-array, $\mathbf{v}_{k}\left(k_{z}\right)$ is:

$$
\mathbf{v}_{k}\left(k_{z}\right)=\left[\begin{array}{c}
e^{-j k_{z} p_{0}} \\
e^{-j k_{z} p_{0}} \\
\vdots \\
e^{-j k_{z} p_{N-1}}
\end{array}\right]
$$

We recall that the purpose of array processing is to linearly combine signals from an array of sensors with a certain weighting in order to find the direction of arrival of the incoming signal. For the incoming plane wave case, with signal $f(t)$, if the inputs on each sensor are shifted such that they align with time, then added and normalized, the array output will be $f(t)$. This process is called delay-and-sum beamforming or conventional beamforming. This concept is illustrated in Figure 2-10.

Going back to the array output function in Equation 2.10, the sensor outputs are shifted by applying:

$$
h_{n}(\tau)=\frac{1}{N} \delta\left(\tau+\tau_{n}\right)
$$


and the result is $y(t)=f(t)$. In the frequency domain, this can be rewritten in vector form:

$$
\mathbf{H}^{T}(\omega)=\frac{1}{N} \mathbf{v}_{k}^{H}\left(\mathbf{k}_{s}\right)
$$

with $\mathbf{k}_{s}$ as the wavenumber for the signal of interest. The frequency-wavenumber response function of the array is the array output to a plane wave of input frequency $\omega$ :

$$
Y(\omega, \mathbf{k})=\mathbf{H}^{T}(\omega) \mathbf{v}_{k}(\mathbf{k})
$$

or

$$
Y(\omega, \mathbf{k})=\frac{1}{N} \mathbf{v}_{k}^{H}\left(\mathbf{k}_{s}\right) \mathbf{v}_{k}(\mathbf{k}) .
$$

The direction of the signal of interest $k_{s}$ is also called the steering direction. Likewise, the steering vector is the array manifold vector in that direction. The frequency-wavenumber response evaluated at different angles in the visible region $0 \leq \theta \leq \pi$ is the beampattern. The beampattern can also be plotted as the power pattern, $\left|B_{\theta}(\theta)\right|^{2}$.

An extension of the unit plane wave frequency-wavenumber response function is a signal with a carrier frequency and some bandwidth $W$. The amplitude $a(t)$ and phase delay $\phi(t)$ are assumed to vary slowly over the interval $\left(0, \tau_{n}\right)$. If signal has bandwidth $\mathrm{W}$, where $\tau_{n}<<1 / W$, then the signal can be described by:

$$
s_{n}(t)=a(t) e^{j 2 \pi f_{c} t+j \phi(t)} .
$$

The signal observed at the $n$th sensor is:

$$
s_{n}(t)=a\left(t-\tau_{n}\right) e^{j 2 \pi f_{c}\left(t-\tau_{n}\right)+j \phi\left(t-\tau_{n}\right)} \approx a(t) e^{j 2 \pi f_{c}\left(t-\tau_{n}\right)+j \phi(t)} .
$$

The delay is approximated by a phase shift, $e^{j \omega \tau_{n}}$.

In order to optimize the performance of the array, the gain and phase at each sensor output can be tuned as a complex weight $w_{n}^{*}$. The weight vector is defined as:

$$
\mathbf{w}^{H}=\left[\begin{array}{llll}
w_{0}^{*} & w_{1}^{*} & \cdots & w_{N-1}^{*}
\end{array}\right]
$$

The weight vector is equivalent to $\mathbf{w}^{H}=\mathbf{H}^{T}\left(f_{c}\right)$. Therefore the array response function 
becomes:

$$
Y(\omega, \mathbf{k})=\mathbf{w}^{H} \mathbf{v}_{k}(\mathbf{k})
$$

For a uniform line array, the weights are equal to: $w_{n}=\frac{1}{N}$. The beampattern for this array setup is consequently:

$$
\begin{array}{r}
B_{\theta}(\theta)=\frac{1}{N} \frac{\sin \left(\frac{N}{2} \frac{2 \pi}{\lambda} \cos (\theta) d\right)}{\sin \left(\frac{1}{2} \frac{2 \pi}{\lambda} \cos (\theta) d\right)}, \\
0 \leq \theta \leq \pi
\end{array}
$$

\subsubsection{Array Design Parameters}

Because array performance depends on a number of factors, we discuss how to optimize the geometry of the array, the complex weighting at the sensor inputs, and the number of sensors based on the UUV acoustic signature we are interested in tracking.

If the array spacing between the elements is greater than $\lambda$ of the signal that is being sampled, then a grating lobe, a lobe of the identical height to the main lobe, will appear. This creates uncertainty into the direction of the peak response of the array. Only prior information can help resolve the true direction of the signal. In order to prevent a grating lobe to appear when the array is steered, the array spacing must be

$$
d \leq \frac{\lambda}{2}
$$

In order to demonstrate the concept of spatial aliasing in the context of this work, Figures 211a-d are the beampatterns for the array spacing used in one of the field experiments. In this setup, the array had 6 elements, with a spacing of $0.075 \mathrm{~m}$. The corresponding spatial aliasing cutoff is $9873 \mathrm{~Hz}$. We show the beampatterns for two different frequencies: $8000 \mathrm{~Hz}$ and $17500 \mathrm{~Hz}$. Although the high frequency beampattern has a narrower beamwidth, spatial aliasing occurs and a grating lobe is present.

Another important consideration is the array angle resolution. The beamwidth of the array determines if two plane waves can be resolved. If the peak of the second beampattern is outside the null of the first pattern, then the other plane wave can be resolved. Thus, the separation must be $\geq 0.5 \mathrm{BW}_{\mathrm{NN}}$ where $\mathrm{BW}_{\mathrm{NN}}$ is the null-to-null beamwidth. For any spacing $d$, and in terms of $\bar{\theta}$ space $(\bar{\theta}=\pi / 2-\theta)$, measured from broadside), the $\mathrm{BW}_{\mathrm{NN}}$ 

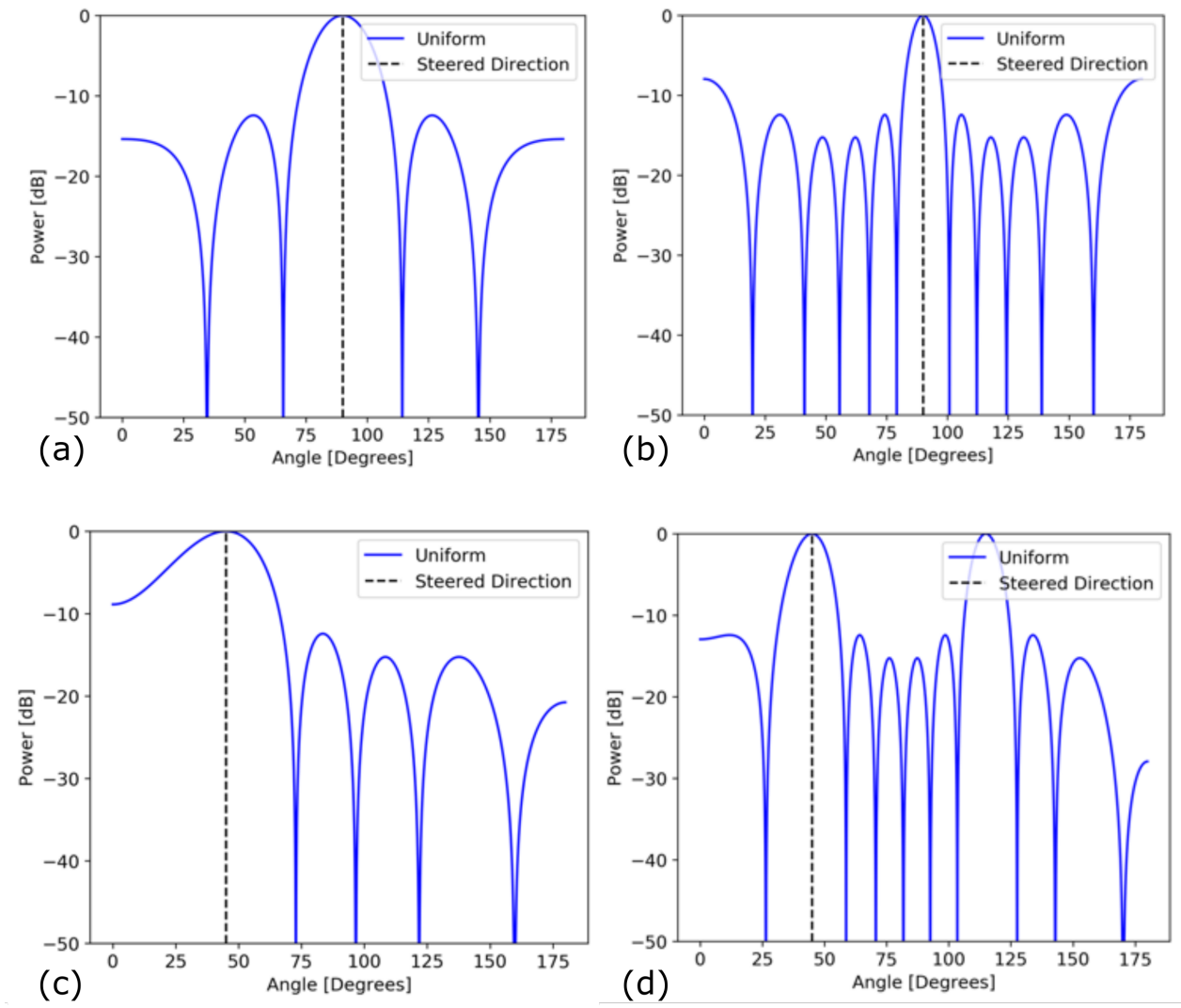

Figure 2-11: Beampatterns are presented for $8000 \mathrm{~Hz}$ steered to (a) broadside and (c) 45 degrees, and $17500 \mathrm{~Hz}$ steered to (b) broadside and (d) 45 degrees.

measured is:

$$
\mathrm{BW}_{\mathrm{NN}}=2 \sin ^{-1}\left(\frac{\lambda}{N d}\right)
$$

One of the goals of array processing is to increase the signal-to-noise ratio of the signal of interest by adding the signal coherently and noise incoherently. The array gain is the ratio of the SNR of the array output compared to the SNR of a single sensor. For a uniform line array, the array gain $A_{w}$ or directivity is

$$
A_{w}=10 \log (N)
$$

By applying non-uniform weighting, such as cosine weighting [72], the sidelobe behavior can be improved at the cost of the main lobe width and directivity. For example, applying cosine weighting to an 11-element array decreases the first sidelobe height from $-13 \mathrm{~dB}$ to $-23.5 \mathrm{~dB}$, but the $\mathrm{BW}_{\mathrm{NN}}$ increased from $2.0 \frac{2}{N}$ to $3.0 \frac{2}{N}$ and directivity decreased from 1 to 


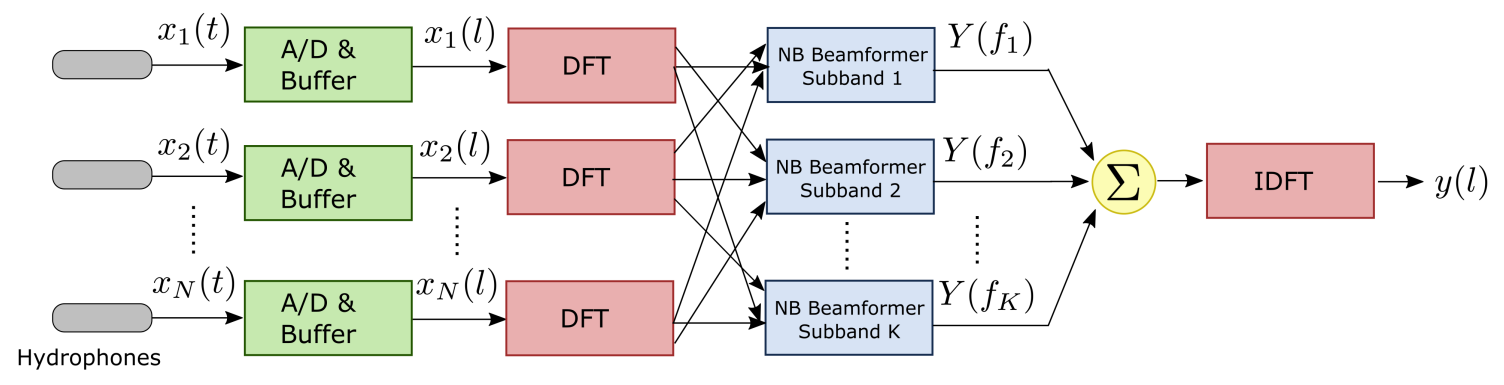

Figure 2-12: Process for frequency-domain snapshot model for broadband beamforming

$0.816[72]$.

\subsubsection{Frequency-domain Snapshot Model for Broadband Beam- forming}

When dealing with a broadband signal, such as the UUV acoustic signature, a discrete Fourier transform can be applied to the sensor input data and then a narrowband beamformer can be implemented on each frequency bin. The narrowband condition is satisfied within each bin.

In practice, the acoustic data from each sensor $n$ is divided into snapshots. Each snapshot is created by applying a sliding window over the data with an observation length $\Delta T$. The snapshots are consequently indexed by $l$. The window length, $\Delta T$, is chosen to be greater than the propagation time across the array. As illustrated in Figure 2-12, after the data from each element is segmented, a discrete Fourier transform (DFT) is performed on each segment of data, $x_{n}(l)$. Following the DFT, a narrowband beamformer is applied to every frequency subband $k$. The array output for each frequency $Y\left(f_{k}\right)$ is summed. Lastly, an inverse discrete Fourier transform is applied to yield the broadband array output $y(l)$.

\subsubsection{Adaptive Beamforming}

Beamforming on a signal of interest is challenging in the presence of noise, because the interfering noise could leak into the sidelobes of the beamformer. One approach to counter the interference is to leverage information on the statistical properties of the signal and background noise. The statistical properties- specifically the signal spatial spectral matrix 
$\mathbf{S}_{\mathbf{X}}$ or the noise spatial spectral matrix $\mathbf{S}_{\mathbf{N}}$ - are estimated from the data and can be incorporated into the beamformer weights. Therefore, by modifying the weights, the directions of interference are suppressed. The spectral matrices update as the data comes in, and the weights adapt. This operation is known as adaptive beamforming.

First, the spectral matrix $\mathbf{S}_{\mathbf{X}}$ is estimated with the sample spectral matrix. One starts with the frequency-domain snapshots for frequency $\omega_{k}: \mathbf{X}_{\mathbf{1}}, \mathbf{X}_{\mathbf{2}} \ldots \mathbf{X}_{\mathbf{l}}$ that have dimensions of $N \times 1$, with the number of elements $N$. It is assumed that the snapshots are independent, identically distributed, Gaussian random vectors. The sample spectral matrix is:

$$
\hat{\mathbf{S}}_{\mathbf{X}}\left(\omega_{k}\right)=\mathbf{C}_{\mathbf{X}}\left(\omega_{k}\right)=\frac{1}{L} \sum_{l=1}^{L} \mathbf{X}_{\mathbf{k}}(l) \mathbf{X}_{\mathbf{k}}^{H}(l) .
$$

Likewise, if we are able to observe the input with no signal present, just noise, $\mathbf{S}_{\mathbf{N}}$ can be estimated:

$$
\hat{\mathbf{S}}_{\mathbf{N}}\left(\omega_{k}\right)=\frac{1}{L} \sum_{l=1}^{L} \mathbf{N}_{\mathbf{k}}(l) \mathbf{N}_{\mathbf{k}}^{H}(l) .
$$

The array gain of the minimum variance distortionless response (MVDR) beamformer uses the sample matrix inversion of $\hat{\mathbf{S}}_{\mathbf{N}}^{-1}$ :

$$
\mathbf{w}_{\mathrm{mvdr}}^{H}=\frac{\mathbf{v}_{\mathbf{s}}^{H} \mathbf{S}_{\mathbf{N}}^{-1}}{\mathbf{v}_{\mathbf{s}}^{H} \mathbf{S}_{\mathbf{N}}^{-1} \mathbf{v}_{\mathbf{s}}}
$$

The number of snapshots must be greater than the number of elements $L \geq N$ for the sample covariance matrix to be invertible. Furthermore, the number of snapshots available for estimating the sample covariance matrix is approximately:

$$
M<B \Delta T
$$

which is the product of the available bandwidth $B$ and the time duration $\Delta T$ that the target is in the array resolution cell [73]. The bandwidth is a function of the speed of sound $c$ and the aperture length $L[73]$ :

$$
B<\frac{c}{8 L}
$$

The time duration that the target is within the resolution cell of the array is a function of 
the bearing rate of the target $\dot{\theta}$, the wavelength $\lambda$, and aperture length $L[73]$ :

$$
\Delta T \approx \frac{\lambda}{L \dot{\theta}}
$$

Therefore, the number of available snapshots becomes [73]:

$$
M<\frac{1}{8 f \dot{\theta}}\left(\frac{\lambda}{L}\right)^{2}
$$

If just the spectral matrix $\mathbf{S}_{\mathbf{X}}$ is available, the total output power is minimized subject to the unity constraint (no distortion) with:

$$
\mathbf{w}_{\mathrm{mvdr}}^{H}=\frac{\mathbf{v}_{\mathbf{s}}^{H} \mathbf{S}_{\mathbf{X}}^{-1}}{\mathbf{v}_{\mathbf{s}}^{H} \mathbf{S}_{\mathbf{X}}^{-1} \mathbf{v}_{\mathbf{s}}} .
$$

When $v_{s}$ aligns with the signal direction, the minimum power distortionless response (MPDR) beamformer is the same as the MVDR beamformer.

\subsubsection{Design of the Acoustic Array System}

The portable underwater modular acoustic array (PUMA) system was designed for multipurpose acoustic data collection in the pool, tank, and field experiments. My design considerations were that the array has an anti-aliasing filter (up to $50 \mathrm{kHz}$ ), the data acquisition supports simultaneous multi-channel measurements, and the hydrophone elements can be arranged in multiple configurations. The overall design will be robust for at-sea deployments, man-portable, and easily programmable. The final setup includes eight hydrophones, digital analog conversion, frequency filtering, and power. A diagram and image of the setup is in Figure 2-13. The array can be configured in a variety of orientations and spacings, as pictured in the pool and field experiments in Figure 2-14. The hydrophones are frequency filtered from $1-30 \mathrm{kHz}$ and can be sampled up to 100,000 samples per second.

\subsubsection{Effect of Temporal and Spatial Aliasing}

In some of the field experiments, temporal and spatial aliasing occurred in the datasets. However, the effect of aliasing was accounted for in the beamforming results and Doppler measurements. The true expected signal of the motor at a hydrophone element $i$ in position, 

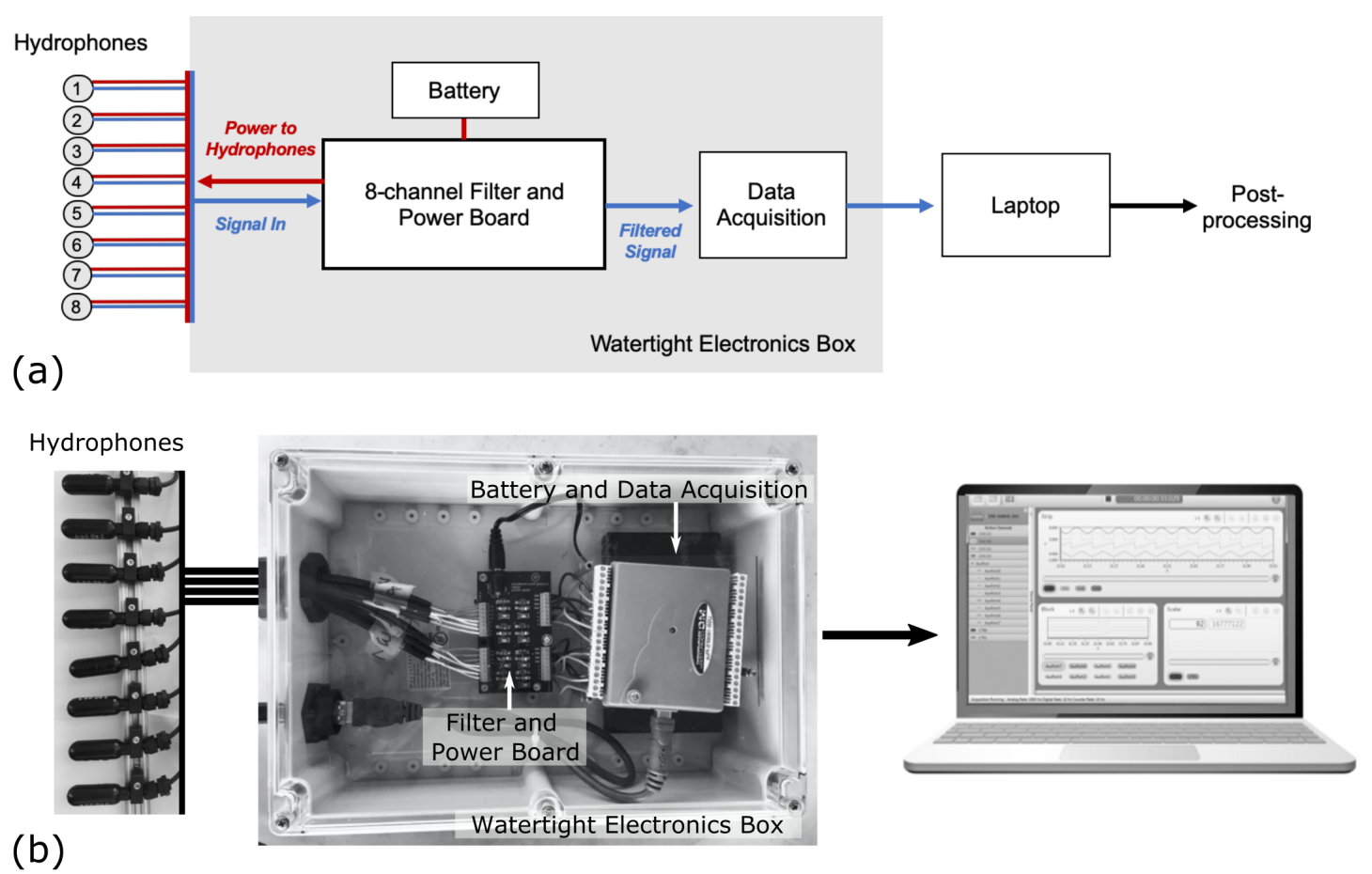

Figure 2-13: The 8-channel data collection method for acoustic measurements is designed to compactly fit in a watertight electronics box.

$p_{i}$, is

$$
x\left(t, p_{i}\right)=A \cos \left(2 \pi f_{\mathrm{PWM}} t-\phi\right)
$$

where the phase delay is $\phi=\frac{2 \pi}{\lambda} \cos (\theta) p_{i}$. Due to temporal aliasing, the reconstructed signal is

$$
x_{r}\left(t, p_{i}\right)=A \cos \left(2 \pi f_{\mathrm{PWM}, \text { aliased }} t+\phi\right),
$$

where $f_{\mathrm{PWM}}$, aliased $=\left(f_{s}-f_{P W M}\right)$ and there is a phase reversal. Therefore, beamforming on a time signal that is aliased will result in a beam at a symmetric angle, $\theta_{r}$, to the intended steering direction, $\theta_{\text {steered }}$, such that $\theta_{\text {steered }}=180-\theta_{r}$. In addition, because the array spacing was $d>\lambda / 2$, a grating lobe is present in the beamforming results. However, with knowledge of the true track of the robot from the vehicle's navigation data, we are able to determine the direction of arrival from the grating lobe.

For one of the experiments, the Doppler shifted frequency of $f_{P W M}$ is above the Nyquist frequency. Thus, when estimating the range rate of the vehicle, one must account for the affect of the aliasing: $f_{\text {aliased, Doppler }}=F_{s}-\left(f_{\mathrm{PWM}}+\Delta f\right)$ or in terms of $f_{\mathrm{PWM} \text {,aliased }}$ : 


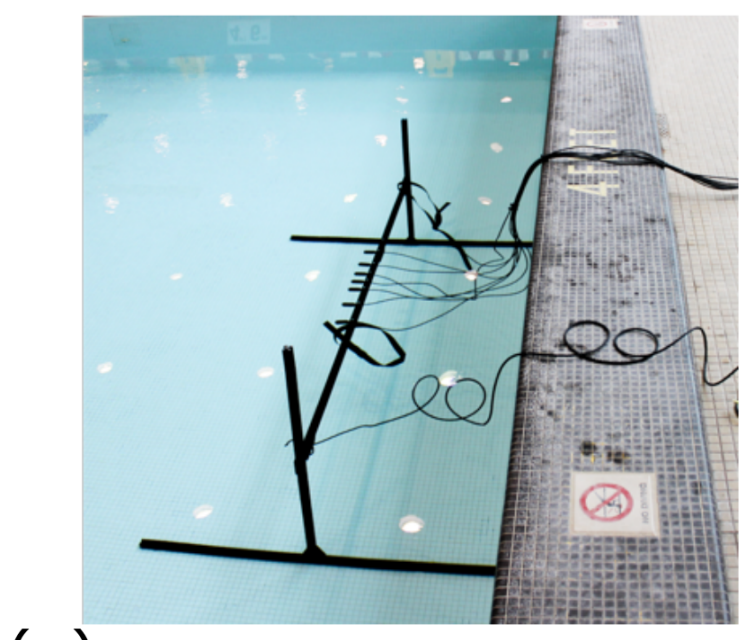

(a)

(b)

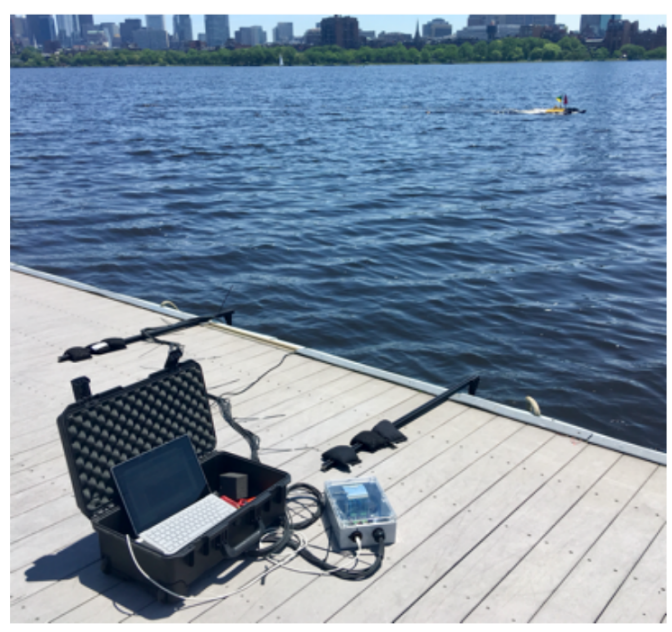

Figure 2-14: Array setup options

$f_{\text {aliased, Doppler }}=f_{\mathrm{PWM} \text {,aliased }}+\Delta f$ where $\Delta f$ is the Doppler shift. When the range rate increases; i.e. when the vehicle is moving away from the array, the true Doppler shift results in a decrease in frequency, but the aliased signal shows an increase in frequency.

\subsection{Pool and Tank experiments}

Based off of the previous work in radiated acoustic noise of vessels, we hypothesize that the motors inside the vehicle's propulsion and stability systems are major contributors of noise. The vehicles selected for study depend on brushless DC motors for propulsion and stability. Previous work in this motor type, and permanent magnet AC synchronous motors generally, has confirmed that the motor emits acoustic noise that is a function of the PWM switching frequency, number of magnetic poles in the motor, blade number, and rotations per minute. In order to confirm that tonal acoustic noise emitting from the robot is related to the motor, we directly measured the acoustic signatures of two unmanned underwater vehicles- the Riptide and Sandshark vehicles- as well as a UUV thruster in isolation - the T200. The purpose of this section is to give a detailed overview of the experimental setups, including images from the actual experiments to further illustrate the setup.

The acoustic signatures of the UUVs are measured in a quiet, controlled pool environment, as shown in Figure 2-15. The vehicle is secured in place, while its propeller rotates 
at different speeds. After varying the speed of the vehicle, the thruster is off and the fins are programmed to change position. With this acoustic data, the relationship between the speed of the vehicle and acoustic tonal features can be derived. Furthermore, tones collected from the fins and thruster are compared to the motor design parameters- such as PWM switching frequency, pole number, and propeller rotation (RPM). In order to derive the acoustic signature of the UUV, the power spectral density is calculated from the time series acoustic data collected on the line array. A standard method for measuring the power spectral density, $S_{X}(f)$, is to use Weiner-Khinchin theorem, which accounts for the tradeoffs of using a longer time sample, to achieve finer spectral resolution, while taking averages of the squared magnitude of the Fourier-transform, $X_{T}(f)$, to account for randomness in the signal, described in Equation 2.43 [74].

$$
S_{X}(f)=\lim _{T \rightarrow \infty} \frac{1}{T} E\left[\left|X_{T}(f)\right|^{2}\right]
$$
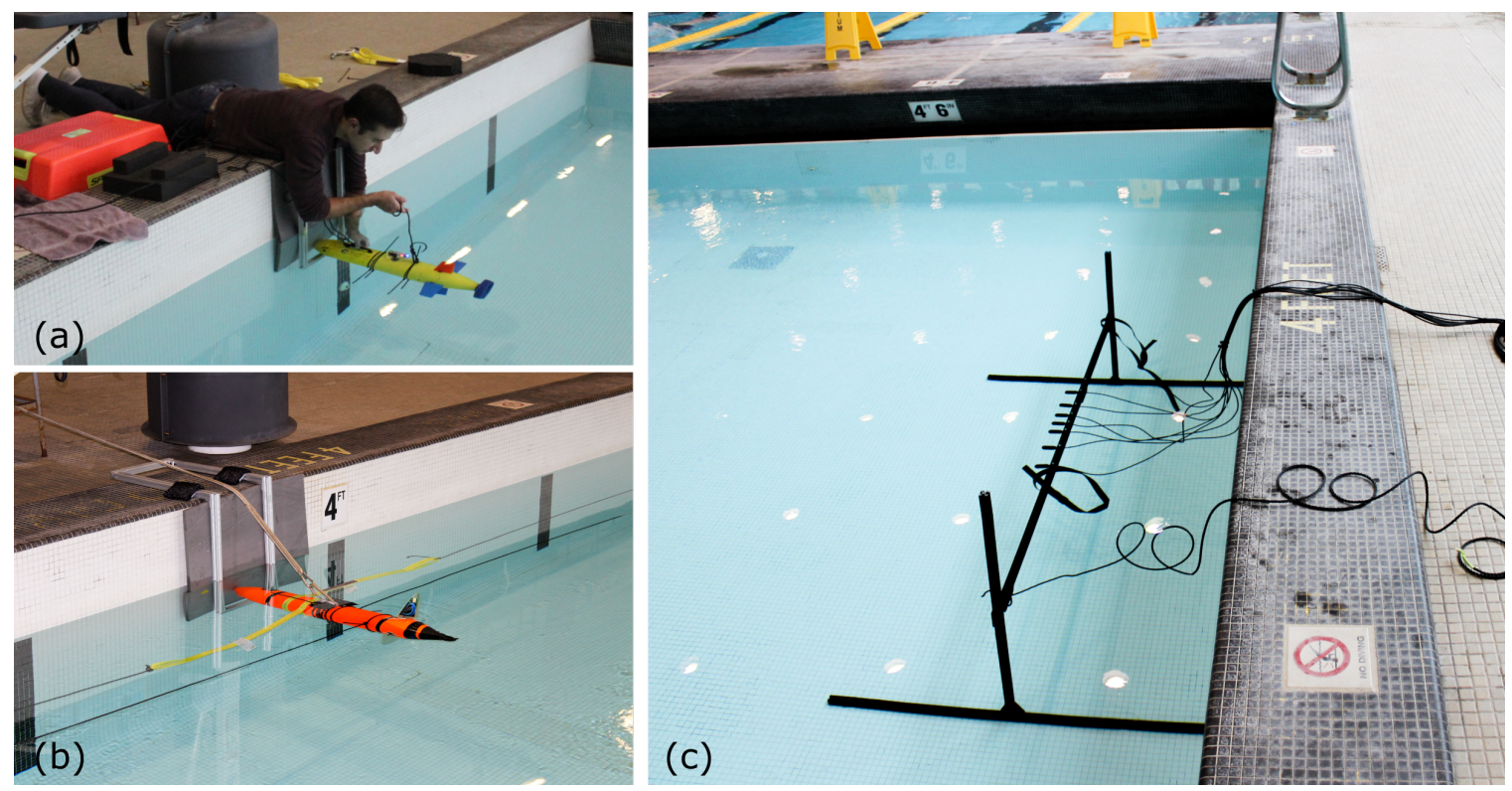

Figure 2-15: Pool experiment setups where the (a) Sandshark and (b) Riptide UUV were held in place on the left wall. On the other end of the pool, (c) an offboard horizontal line array collected acoustic measurements.

To further isolate noise in the UUV emitting from the thruster motor, we conducted a motor isolation experiment in a tank, pictured in Figure 2-16. The UUV thruster is mounted to the tank and the acoustic measurements are taken offboard. 
Finally, I will measure the acoustic noise of the UUV in the Charles River, where the acoustic array is mounted to the pavilion dock and the UUV performs a typical autonomous mission. Together with pool, tank, and river data of two different micro-UUVs, I will be able to draw conclusions into the strong tonal features of the UUVs - what they have in common, where the tones come from, and how the tones change with vehicle speed.
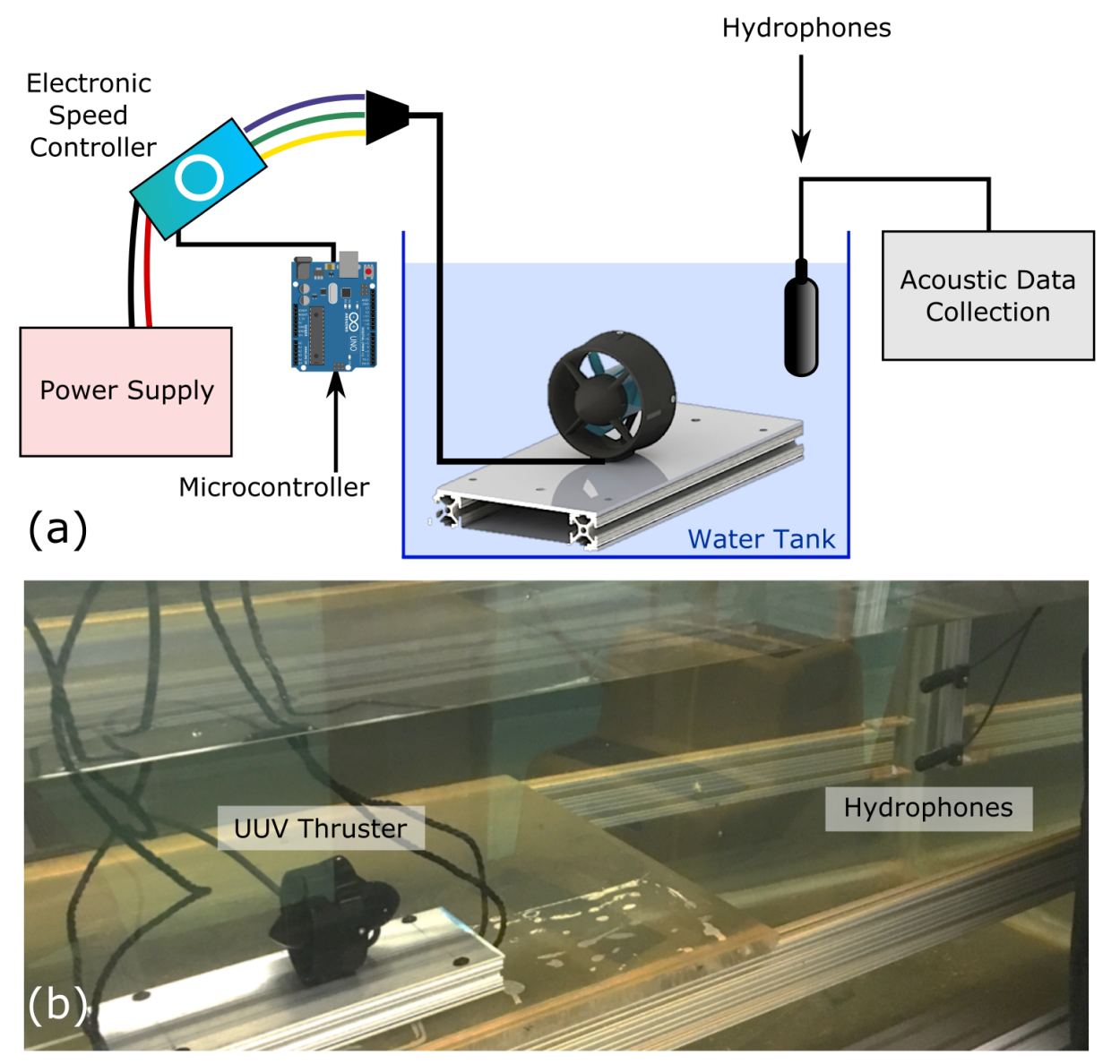

Figure 2-16: (a) Diagram of the tank experiment for collecting acoustic noise from the thruster is presented. (b) The acoustic data is collected from two hydrophones. The thruster is mounted to the bottom of the tank.

\subsection{Target Motion Analysis}

In target motion analysis (TMA), or passive localization, noisy and remote measurements are fused to localize and track a target with a state estimation technique. In this section, we provide an overview of the key concepts in target tracking, as well as justification for 
our approach to implement an unscented Kalman filter. To demonstrate these concepts, derivations of the batch processing least squares estimator, discrete-time linear Kalman filter, and Unscented Kalman filter are included.

A general definition of estimation is the "process of inferring the value of a quantity of interest from indirect, inaccurate, and uncertain observations." [3]. The goal of target motion analysis is to estimate the state of the target with passive sensors, which could be acoustic or electromagnetic. The state of a mechanical system, such as a robot, is the position and velocity of the platform. More generally, the state is a set of variables that fully describe the target's dynamical system, such that future states can be predicted.

Tracking is defined as estimating the state of the target with remote measurements. In our case, the UUV state is the position and velocity (depth is neglected), which is estimated from passive and remote acoustic measurements. The observation or measurement equations transform the state variables of the vehicle into the expected acoustic measurements of bearing, bearing rate, range rate, and RPM. It is important to note, that these measurement equations are non-linear. In this work, we also leverage previous research done in UUV navigation. Navigation, which also involves estimating the state of the vehicle, differs from tracking in the sensor location: the sensors are on its own platform, not remote. Advances in UUV navigation are summarized in Paull et al. [31], Leonard et al. [28], and Kinsey et al $[75]$.

For the UUV tracking application, we assume the robot moves at a constant-velocity. There are two main approaches for tracking a constant-velocity target with non-linear measurement equations: recursive Bayesian estimation and batch processing least-squares estimation, which are described in detail in Bar-Shalom et al. [3] and Gibbs et al [76]. In the field experiments, the vehicle moved in a circular pattern. As a consequence, we have mismatch between the vehicle's actual behavior and the constant-velocity model. To resolve the mismatch, the recursive Bayesian technique - the Unscented Kalman filter- was selected since it can account for model mismatch with process noise error. The Unscented Kalman filter (UKF) was selected over other nonlinear state estimation methods such as the extended Kalman filter and Particle Filter for the following reasons: the UKF possesses better stability over the extended Kalman filter [77,78]; and the UKF runs more efficiently than the Particle Filter [79,80]. Additional techniques that could be considered for this application where the vehicle behavior is "highly maneuverable" (or not moving at 
a constant velocity) include: applying interacting multiple model-based trackers, particle filters, hidden Markov models, and using multiple independent EKFs with different range estimates [81-85].

Tracking the UUV from bearing, Doppler, and RPM measurements is compared to traditional target motion analysis which only involves the measurements of bearing and range rate. The significance of adding a unique measurement is an increase in the observability of the robot position from the measurements available. Observability is the ability to find a unique tracking solution. If the observing platform moves at a constant velocity, the target state estimate cannot be determined with bearing-only measurements because there are an infinite number of values for the state estimate that yield the same observations. Therefore, the observing platform must "outmaneuver" the target [86]. There has been extensive research in passively tracking an emitting source from bearing-only measurements including, but not limited to the following topics: bearing-only TMA with highly maneuverable targets [82-85], cooperative localization [87,88], optimal observer trajectory [89-94], and a comparison of polar and cartesian coordinates [95].

When bearing and dopplerized radiated frequencies are both available, a unique target motion analysis solution can be determined without any maneuver of the observer platform [96]. Therefore, a unique track solution of the target can be realized from a stationary line array. Implementing Doppler-bearing [97-102] and Doppler-only [103,104] TMA has been thoroughly researched. Bearing-Doppler TMA is used as a baseline case for tracking the UUV.

\subsubsection{Batch Processing Least-squares Estimation}

For context, we introduce the non-linear least squares approach of estimating the position of a constant velocity target in discrete-time with bearing-only measurements. The target state includes the initial position of $x_{0}$ and $y_{0}$ and velocity components of $v_{x}$ and $v_{y}$ :

$$
\vec{x}=\left[\begin{array}{llll}
x_{0} & y_{0} & v_{x} & v_{y}
\end{array}\right]^{T} .
$$


The position of the robot at time $t_{k}$ is:

$$
\begin{aligned}
& x(k)=x_{0}+v_{x} t_{k} \\
& y(k)=y_{0}+v_{y} t_{k}
\end{aligned} .
$$

Over time, the observer platform takes bearing measurements which have a noise component, $w(k)$, and a truth component, $h(k, \vec{x})$, which together make

$$
z(k)=h(k, \vec{x})+w(k)
$$

Bearing is related to the target and observer position, $x_{\text {array }}$ and $y_{\text {array }}$, through geometry in the non-linear function:

$$
h(k, \vec{x})=\tan ^{-1} \frac{y(k)-y_{\text {array }}(k)}{x(k)-x_{\text {array }}(k)} .
$$

Therefore, the cost function for finding the target state from a series of measurements is the log-likelihood function:

$$
\lambda(\vec{x})=\frac{1}{2 r} \sum_{k=1}^{n}[z(k)-h(k, \vec{x})]^{2} .
$$

This multi-variable cost function for finding the target state can be minimized using NewtonRhapson or quasi-Newton methods [3]. The observability is evaluated by deriving the Cramer-Rao lower bound, the covariance matrix of the target state estimate and function of the Fisher information matrix (FIM) [3]:

$$
E\left[(\hat{x}-x)(\hat{x}-x)^{\prime}\right] \geq J^{-1}
$$

The target estimate is $\hat{x}$, the true target state is $x$, and the Fisher information matrix is $J$. In practice, true target state is not known, so evaluation is done at the estimate (by finding the gradient of the Log-likelihood function) [3]. A key difference between this state estimation technique and the Kalman filter is that the latter accounts for process noise. 


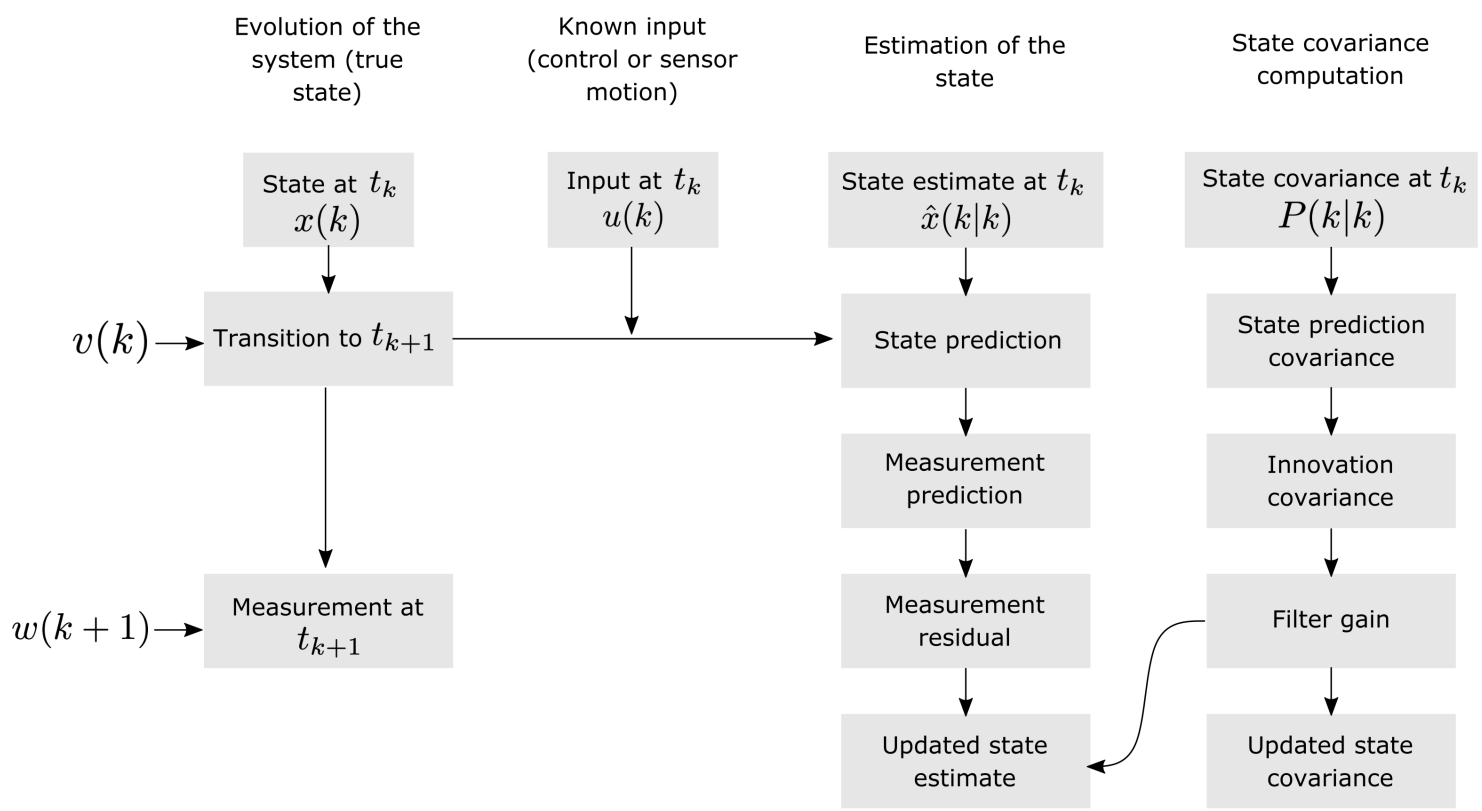

Figure 2-17: Kalman filter process, adapted from Bar-Shalom et al. [3]

\subsubsection{Kalman Filter}

Before describing the unscented Kalman filter implementation, we provide an overview of the fundamental Kalman filter framework for a discrete-time linear dynamic system. The core idea of this method is to use a predict and update cycle to estimate the state of the dynamical system from sensor measurements:

1. The state for the next time step, and its corresponding expected measurements, are predicted.

2. Measurements are taken, and the state is updated.

At the update step, a filter gain is applied to the residual of the expected measurement and the actual measurement. The filter gain is calculated from the covariances. A "large" gain corresponds to high accuracy of the measurement and low accuracy of the state prediction. Likewise, a "small" gain corresponds to high accuracy of the state prediction and low accuracy of the measurement. A flow-diagram of the Kalman filter process is in Figure 217. In the derivation of the Kalman filter process, the key definitions which correspond to blocks in the flow-diagram are bolded and italicized.

First, we assume we have a known initial state and covariance, $E\left[x(0) \mid Z^{0}\right]=\hat{x}(0 \mid 0)$ and $\operatorname{cov}\left[x(0) \mid Z^{0}\right]=P(0 \mid 0)$. Furthermore, we assume the measurement noise $w(k)$ and 
process noise $v(k)$ sequences are zero-mean, white, and mutually uncorrelated. Consequently, $v(k)$ and $w(k)$ are independent of the measurements: $E\left[v(k) \mid Z^{k}\right]=E[v(k)]=0$ and $E\left[w(k) \mid Z^{k}\right]=E[w(k)]=0$.

The dynamic equation, or process model, describes how the current state evolves to the next state after a time-step (Transition to $\left.\boldsymbol{t}_{k+1}\right)$ :

$$
\begin{array}{r}
x(k+1)=F(k) x(k)+G(k) u(k)+v(k) \\
\text { where } k=0,1, \ldots
\end{array}
$$

and $x$ is the state vector of $n \times 1$ (where $n$ is the number of states):

$$
x=\left[\begin{array}{llll}
x_{1, k} & x_{2, k} & x_{3, k} & \cdots
\end{array}\right]^{T}
$$

The state variables are Gaussian random variables, $\mathcal{N}\left(\mu_{x}, \sigma_{x x}\right)$. The $u$ is a known input vector (deterministic) of $m \times 1$ :

$$
u=\left[\begin{array}{llll}
u_{1, k} & u_{2, k} & u_{3, k} & \cdots
\end{array}\right]^{T} .
$$

The $v(k)$ is the process noise, which represents the uncertainty of the state transition model. The process noise is a sequence of zero-mean Gaussian noise with dimensions $n \times 1$ and its covariance is $Q(k): E\left[v(k) v(k)^{T}\right]=Q(k)$. The matrix $G(k)$ converts the input vector, with dimensions of $n \times m$. The state transition model is $F(k)$, which is a square matrix of size $n \times n$.

For a linear transformation of random variables, $Y=a X+b$, the variance is $\operatorname{var}(Y)=$ $a^{2} \operatorname{var}(X)$ and the mean is $E[Y]=a E[X]+b$. Also the probability density function of $Y$ is: $f_{Y}(y)=\frac{1}{|a|} f_{X}\left(\frac{y-b}{a}\right)$. These concepts can be applied to the linear dynamic equation, Equation 2.50. The conditional probability $P\left(x_{k} \mid u_{k}, x_{k-1}\right)$ is conditioned on $u_{k}$, the control inputs which are already deterministic, and $x_{k-1}$, which is a random variable. Considering $v(k)$ is Gaussian, it follows for $P\left(x_{k} \mid u_{k}, x_{k-1}\right)$ to also be Gaussian. Looking at the general expression $Y=a X+b$, in this case "b" or the shift is $F(k) x(k)+G(k) u(k)$ and "a" is 1 . Also the expectation and variance of $v(k)$ are $E[v(k)]=0$ and $\operatorname{var}(v(k))=Q(K)$. Because a Gaussian distribution can be described by its mean and variance, the state transition 
probability $P\left(x_{k} \mid u_{k}, x_{k-1}\right)$ is consequently:

$$
P\left(x_{k} \mid u_{k}, x_{k-1}\right)=\mathcal{N}(F(k-1) x(k-1)+G(k-1) u(k-1), Q(k-1)) .
$$

The measurement equation transforms the observable measurements into the state variables:

$$
\begin{array}{r}
z(k)=H(k) x(k)+w(k) \\
\text { where } k=1,2,3 \ldots
\end{array} .
$$

The measurement noise $w(k)$ is a sequence of zero-mean Gaussian noise with covariance $E\left[w(k) w(k)^{T}\right]=R(k)$ and the observation model is $H(k)$. The likelihood probability of $P\left(z_{k} \mid x_{k}\right)$, comes from applying a linear transformation to random variable $w_{k}$ :

$$
P(z(k) \mid x(k))=\mathcal{N}(H(k) x(k), R(k))
$$

The state estimate is the expected value of $x(j)$ conditioned on the sequence of observations, $Z^{K}$ :

$$
\hat{x}(j \mid k)=E\left[x(j) \mid Z^{K}\right]
$$

where $Z^{K}=\{z(i), i \leq k\}$. The predicted state is therefore:

$$
\hat{x}(k+1 \mid k)=E\left[x(k+1) \mid Z^{k}\right]=E\left[F(k) x(k)+G(k) u(k)+v(k) \mid Z^{k}\right] .
$$

Because $v(k)$ is white and zero-mean, $u(k)$ is deterministic, and $\hat{x}(k \mid k)=E\left[x(k) \mid Z^{k}\right]$, this simplifies to (the state prediction):

$$
\hat{x}(k+1 \mid k)=F(k) \hat{x}(k \mid k)+G(k) u(k) .
$$

From Equation 2.58, the predicted measurement is:

$$
E\left[z(k+1) \mid Z^{k}\right]=E\left[H(k+1) x(k+1)+w(k+1) \mid Z^{k}\right]
$$

which simplifies to:

$$
\hat{z}(k+1 \mid k)=H(k+1) \hat{x}(k+1 \mid k)
$$


because measurement noise is zero-mean, $E[w(k)]=0$ and Equation 2.56.

The estimation error is defined as $\tilde{x}(j \mid k)=x(j)-\hat{x}(j \mid k)$. Therefore, the state predicted error is then:

$$
\tilde{x}(k+1 \mid k)=x(k+1)-\hat{x}(k+1 \mid k)
$$

which is also equal to:

$$
\begin{array}{r}
\tilde{x}(k+1 \mid k)=F(k) x(k)+G(k) u(k)+v(k)-F(k) \hat{x}(k \mid k)-G(k) u(k) \\
\tilde{x}(k+1 \mid k)=F(k) x(k)+v(k)-F(k) \hat{x}(k \mid k) \\
\tilde{x}(k+1 \mid k)=F(k) \tilde{x}(k \mid k)+v(k)
\end{array}
$$

Note, the definition of an auto-covariance matrix of real random vectors $X=\left[X_{1}, X_{2}, \ldots X_{m}\right]^{T}$ is:

$$
K_{X X}=\operatorname{cov}(X, X)=E\left[(X-E[X])(X-E[X])^{T}\right]=E\left[X X^{T}\right]-E[X] E[X]^{T}
$$

Because $\hat{x}(k+1)$ is deterministic, the state prediction covariance is:

$$
P_{x x}(k+1 \mid k)=\operatorname{cov}\left(x(k+1) \mid Z^{K}\right)=\operatorname{cov}\left(\tilde{x}(k+1 \mid k) \mid Z^{K}\right)
$$

This can also be written as:

$$
P_{x x}(k+1 \mid k)=E\left[\left(x(k+1)-E\left[x(k+1) \mid Z^{k}\right]\right)\left(x(k+1)-E\left[x(k+1) \mid Z^{k}\right]\right)^{T} \mid Z^{k}\right]
$$

Since $\hat{x}\left(k+1 \mid Z^{k}\right)=E\left[x(k+1) \mid Z^{k}\right]$ and $E\left[\tilde{x}(k+1) \mid Z^{k}\right]=E\left[x(k+1)-\hat{x}(k+1) \mid Z^{k}\right]$ the state prediction covariance becomes:

$$
\begin{array}{r}
P_{x x}(k+1 \mid k)=E\left[\left(x(k+1)-\hat{x}\left(k+1 \mid Z^{k}\right)\right)\left(x(k+1)-\hat{x}\left(k+1 \mid Z^{k}\right)\right)^{T} \mid Z^{k}\right] \\
P_{x x}(k+1 \mid k)=E\left[\tilde{x}(k+1 \mid k) \tilde{x}(k+1 \mid k)^{T} \mid Z^{k}\right]
\end{array}
$$

Using Equation 2.50 and Equation 2.58, the state prediction covariance becomes:

$$
P_{x x}(k+1 \mid k)=F(k) P(k \mid k) F(k)^{T}+Q(k)
$$

because $G(k) u(k)$ cancels, the matrix distributivity property applies, and the cross-terms 
cancel [3]. Following similar steps, the measurement prediction covariance is:

$$
S(k+1)=H(k+1) P(k+1 \mid k) H(k+1)^{T}+R(k+1)
$$

Using the covariance between the measurement and state, the filter gain which is a function of the measurement and process covariance matrices is:

$$
W(k+1)=P(k+1 \mid k) H(k+1)^{T} S(k+1)^{-1} .
$$

Therefore the updated state estimate is:

$$
\hat{x}(k+1 \mid k+1)=\hat{x}(k+1 \mid k)+W(k+1) v(k+1)
$$

with the measurement residual equation to:

$$
v(k+1)=z(k+1)-\hat{z}(k+1 \mid k)=\tilde{z}(k+1 \mid k) .
$$

Lastly, the updated state covariance is:

$$
P(k+1 \mid k+1)=P(k+1 \mid k)-W(k+1) S(k+1) W(k+1)^{T} .
$$

The Kalman filter stability depends on observability. If no information about a state can be learned through the observation equations, regardless of the number of observations, then the filter estimate for the state will not converge on the right tracking solution [105]. For linear time-invariant systems, observability can be assessed by finding the rank of the observability matrix which is a function of the state transition matrix $F$ and the observation matrix $H[105]$.

\subsubsection{Unscented Kalman Filter}

The traditional Kalman filter holds for linear systems with Gaussian distributed errors. In the case of passive acoustic tracking, however, the observation equations are non-linear. For instance, the mapping from array bearing (measurement) to the vehicle position (target state) involves the tangent function. The extended Kalman filter is commonly used for 
handling non-linear systems. However, this method is challenging to implement and unstable for highly non-linear systems [77]. Therefore, in this work we implement the unscented Kalman filter (UKF), which is presented in Julier et al [77]. This filter provides superior stability than the standard EKF.

Although the UKF has the same core structure of the fundamental Kalman filter with a predict and update process, its key differentiating feature is the unscented transform function. This function is used to parameterize the probability distributions of random variables (i.e. the state variables) as Gaussian when the variables are passed through a non-linear function (i.e. the state transition function). The underlying principle of this approach is that it is simpler to approximate a Gaussian distribution than approximating a nonlinear function.

In the unscented transformation, an n-dimensional random variable $x$ of mean $\bar{x}$ and covariance $P_{x x}$ is represented with $2 n+1$ weighted sigma points:

$$
\begin{array}{r}
\chi_{0}=\bar{x} \\
\chi_{i}=\bar{x}+\left(\sqrt{(n+\lambda) P_{x x}}\right)_{i} \\
\text { for } i=1 \ldots n . \\
\chi_{i}=\bar{x}-\left(\sqrt{(n+\lambda) P_{x x}}\right)_{i} \\
\text { for } i=(n+1) \ldots 2 n
\end{array}
$$

The corresponding weights for the covariance $W_{i}^{c}$ and means $W_{i}^{m}$ are:

$$
\begin{array}{r}
W_{0}^{m}=\lambda /(n+\lambda) \\
W_{0}^{c}=\lambda /(n+\lambda)+1-\alpha^{2}+\beta \\
W_{i}^{m}=W_{i}^{c}=1 / 2(n+\lambda) \\
W_{i+n}^{m}=W_{i+n}^{c}=1 / 2(n+\lambda)
\end{array}
$$

where $\lambda$ accounts for spread of the sigma points about the mean with parameter $(\alpha)$, prior knowledge of the distribution is accounted for in $\beta$, and $\kappa$ is a secondary scaling parameter as described in Merwe et al. [106]:

$$
\lambda=\alpha^{2}(n+\kappa)-n
$$


Next, the points follow the process of the unscented transform. The points $\chi_{i}$ are passed through the function (i.e. the observation function) to yield:

$$
\gamma_{i}=f\left[\chi_{i}\right]
$$

The mean is produced by calculating the weighted average of the transformed points:

$$
\bar{y}=\sum_{i=0}^{2 n} W_{i} \gamma_{i} .
$$

Then the covariance is calculated as:

$$
P_{y y}=\sum_{i=0}^{2 n} W_{i}\left\{\gamma_{i}-\bar{y}\right\}\left\{\gamma_{i}-\bar{y}\right\}^{T} .
$$

In terms of applying the unscented transform into the predict and update steps of the Kalman filter, the process is as follows.

First, the sigma points $\chi$ and their respective weights $W^{m}, W^{c}$ are found for the ndimensional state variable $x$ and its covariance $P_{x x}$ :

$$
\begin{gathered}
\chi=f_{\text {sigma points }}\left(x, P_{x x}\right) \\
W^{m}, W^{c}=f_{\text {weights }}(n) .
\end{gathered}
$$

The process model or state transition function $f(x)$ transforms the set of sigma points $\chi$ to:

$$
\gamma=f(\chi)
$$

The unscented transformation is applied to the sigma points to calculate the mean and covariance of the predicted state.

$$
\begin{array}{r}
\bar{x}=\sum_{i=0}^{2 n} W_{i}^{m} \gamma_{i} \\
\bar{P}_{x x}=\sum_{i=0}^{2 n} W_{i}^{c}\left(\gamma_{i}-\bar{x}\right)\left(\gamma_{i}-\bar{x}\right)^{T}+Q
\end{array}
$$

where $Q$ is the process noise. Next, the measurement is predicted from $\gamma$ :

$$
Z=h(\gamma)
$$


where $h(\gamma)$ is the (non-linear) measurement function. Then the unscented transform is used to find the mean and covariance from the sigma points $Z$ :

$$
\begin{array}{r}
\bar{z}=\sum_{i=0}^{2 n} W_{i}^{m} Z_{i} \\
\bar{P}_{z z}=\sum_{i=0}^{2 n} W_{i}^{c}\left(Z_{i}-\bar{z}\right)\left(Z_{i}-\bar{z}\right)^{T}+R
\end{array}
$$

where $R$ is the measurement noise matrix. The state estimate is updated with an incoming measurement $z$. The measurement residual is:

$$
v=z-\bar{z} .
$$

The filter gain is derived for updating the state estimate:

$$
K=P_{X Z} P_{Z}^{-1}
$$

where

$$
P_{X Z}=\sum_{i=0}^{2 n} W_{i}^{c}\left(\gamma_{i}-\bar{x}\right)\left(Z_{i}-\bar{z}\right)^{T} .
$$

Finally, the filter gain is applied to the measurement residual to yield the updated state estimate and its covariance:

$$
\begin{array}{r}
x=\bar{x}+K y \\
P=\bar{P}-K P_{Z} K^{T}
\end{array} .
$$




\section{Chapter 3}

\section{An Acoustic Remote Sensing Method for High-precision Propeller Rotation and Speed Estimation of UUVs}

\subsection{Introduction}

In the past decade, unmanned underwater vehicle (UUV) technology has significantly improved in the areas of navigation, sensing, and autonomy. With new developments in navigation, UUVs have become capable of traveling under moving ice, which was previously considered an impossible task, giving scientists critical data to understand climate change in ice-covered seas [107]. During Deepwater Horizon, vehicles equipped with multi-sensor packages, including a mass spectrometer, were used to assess the impact of oil spills [108]. Research in optimal path planning and swarm cooperation has enabled vehicles to efficiently collect environmental data on large spatial and temporal scales in the ocean, which has been critical for weather modeling [109]. In defense applications, UUVs have historically played a role in mine countermeasures. With advancing technology, UUVs are tracking submarines with active sonar [110], surveilling the ocean for intelligence missions with optimal path planning for energy consumption and avoidance of fishing nets [111], and delivering mines 
strategically which requires precise navigation [112]. The vehicles are performing more offensive missions, and so countering UUVs from acting on their malicious intent has become a new national security priority [24-26]. Existing passive acoustic defense systems are challenged by detecting and tracking these vehicles.

In general, passive acoustic monitoring (PAM) is important for a range of applications from surveilling marine protected areas to harbor security. PAM applied to the radiated noise of vessels- ships [44,45,113], submarines [114], and torpedoes [1] - and even divers [115-117] has been thoroughly investigated. As discussed in Urick [1], the prominent sources of radiated noise in vessels are machinery and propeller noise, which can be analyzed for classifying the target and estimating target speed. The continuous spectrum of propeller cavitation noise is amplitude modulated at the propeller shaft rotation speed or at the propeller blade rate frequency. A method to identify these fundamental frequencies and their harmonics is the DEMON (Detection of Envelope Modulation on Noise) algorithm $[43-45,118]$. In the low frequency domain, propeller noise also consists of discrete spectral components at the blade rate frequency and its harmonics [1]. Identifying small boat signatures in noisy environments by applying DEMON analysis and measuring low frequency blade lines has been demonstrated [44,45,113]. For submarines, Dixon et al. discusses the directivity pattern of the blade rate sound and how the unique turn-per-knot ratio (mapping of the rotational speed of the propeller to the underway speed) of a vessel can be used for estimating speed [114].

When using this method in practice for classifying and tracking UUVs, one most consider some important aspects of propeller noise which affect the DEMON algorithm performance and differ from machinery noise: the characteristic radiation pattern is weaker in the foreand-aft directions and the SNR strongly depends on speed and depth [1]. Another challenge for DEMON analysis is the selection of the propeller noise passband filter, which can significantly affect the algorithm's performance. The ideal passband filter varies widely between vessels and is chosen generally based on trial and error [45]. Passively tracking vessels by low frequency blade lines and propeller noise is challenging in a noisy and dynamic environment such as the shallow waters of harbors. Harbors are rich with noise sources: pile driving, ship traffic, motor vehicle traffic on nearby bridges, and construction. The cutoff frequency for a shallow water duct should also be taken into consideration.

Passively detecting and tracking UUVs from DEMON spectra, and more generally, ra- 
diated noise, is a relatively unexplored research area in comparison to other marine vessels. There has been an initiative (summarized in Holmes et al.) to understand and mitigate unwanted noise in mid- to low-frequencies (below $10 \mathrm{kHz}$ ) that interferes with onboard acoustic sensors [119]. The authors discuss how the field fluctuations in the magnetic pole gap between the stator and rotor inside a motor lead to significant monopole vibration of the motor housing [119]. In the field, Gebbie et al. analyzed the angular dependence of radiated noise from an underway REMUS-100 UUV via a bottom-mounted horizontal line array in a quiet ocean environment [120]. The authors were able to track the UUV by a single tone of $1065 \mathrm{~Hz}$, which they attributed to originate from the propulsion system. Zhang et al. discussed how the Doppler effect on symmetric spectral lines around the switching frequency of an inverter-fed motor can be measured to estimate radial velocity [121]. However, previous work in radiated noise of UUVs does not pinpoint the causes of spectral noise to the vehicle's motor design parameters or estimate the speed of the vehicle.

In order to isolate and characterize motor noise, we focus on brushless DC (BLDC) motors because they are prevalent in UUV propulsion systems - including the REMUS100/600/6000, Sentry, Iver2, Dorado-class [47], and Autosub [49] - for their reliability, efficiency, and low-noise [34]. Various design aspects for these motors- the pole number, motor operating frequency, the pulse width modulated (PWM) switching frequency $\left(f_{\mathrm{PWM}}\right)$, and the natural mechanical vibration modes of the whole system- contribute to strong tonal acoustic features [69]. Several groups have confirmed through experimentation and modeling that $f_{\mathrm{PWM}}$ and its harmonics, especially $2 f_{\mathrm{PWM}}$, are dominant sources of noise $[63,64,66-70]$. In general, to address noise mitigation in motors, several techniques include: reducing cogging torque ripple through magnetic pole design [122], randomized PWM [71], and modifying the commutation sequence [123]. However, these design changes are not without tradeoffs. Islam et al. points out that for the mass-manufactured BLDC motor, minimizing cogging torque ripple increases overall cost, complexity of the stator and rotor magnet construction as well as the potential loss of output torque. Lo et al. summarizes limitations of altering $f_{\mathrm{PWM}}$ to reduce noise. A randomized PWM signal, which results in flat, broadband noise, may eliminate a strong tone but then increase the probability of aligning with other mechanical resonances. For some applications, it is sufficient to move $f_{\mathrm{PWM}}$ outside of the audible range. However, increasing $f_{\mathrm{PWM}}$ leads to higher switching loss in MOSFETs [69]. 
Building off of previous work in both UUV and BLDC motor noise, we describe a passive acoustic, high precision method for estimating the micro-UUV's propeller rotation frequency, which outperformed the DEMON algorithm in field experiments with highly variable noise and shallow water. Although the analysis of this work is focused on micro-UUVs, this method applies to other robotic platforms which are powered by mass-manufactured BLDC motors. First, the acoustic signatures of the two micro-UUVs (Section 3.3.1) and an isolated thruster (Section 3.3.2) were measured and DEMON analysis performed. From this analysis, the sources of noise from the BLDC motor inside the propulsion systems are identified. Measuring the motor noise as a method for estimating UUV propeller speed was evaluated in field experiments with the two micro-UUVs (Section 3.3.4).

\subsection{Methodology}

\subsubsection{Overview of the Sandshark and Riptide Micro-UUVs}

The BAE Systems Riptide (Mk1 version) and the General Dynamics Mission Systems Bluefin Sandshark micro-UUVs, pictured in Figure3-1, were selected for study. The tailcone section of the Sandshark consists of a thruster, fin actuators, battery, altimeter, and inertial navigation system (INS). For propulsion, the thruster includes a motor controller and BLDC motor (Anaheim Automation BLWR173S-24V-2000) which operates with a PWM frequency between $15-20 \mathrm{kHz}$ and 8 poles, producing a top speed of $2.5 \mathrm{~m} / \mathrm{s}$. The vehicle dynamics for pitch, roll, and heading are controlled by three fins actuated by a BLDC servomotor (Maxon EC-max 16), which is commanded by a PWM signal. The Riptide vehicle has a similar configuration as the Sandshark, but a notable difference is its reported top speed of $10 \mathrm{kts}$ or $5.14 \mathrm{~m} / \mathrm{s}$. The thruster consists of a BlueRobotics M200 motor, which is a BLDC 3-phase motor. The BlueRobotics Basic 30A ESC controller (r1) creates a 3-phase, PWM signal to power the motor at the desired rotations per minute. This PWM frequency is at $17,857 \mathrm{~Hz}$. To isolate acoustic noise related to the motor, the BlueRobotics T200 thruster (electronic speed controller, BLDC motor, and propeller) was measured separately from the entire UUV system. This thruster was selected because the Riptide propulsion system is powered by an earlier version of this motor. In this updated version, the T200 motor is a brushless outrunner BLDC motor with 14 permanent magnetic poles on the rotor 
and 12 stator coils. This motor is controlled with the BlueRobotics Basic ESC (r3), which operates with a PWM frequency of $24 \mathrm{kHz}$.

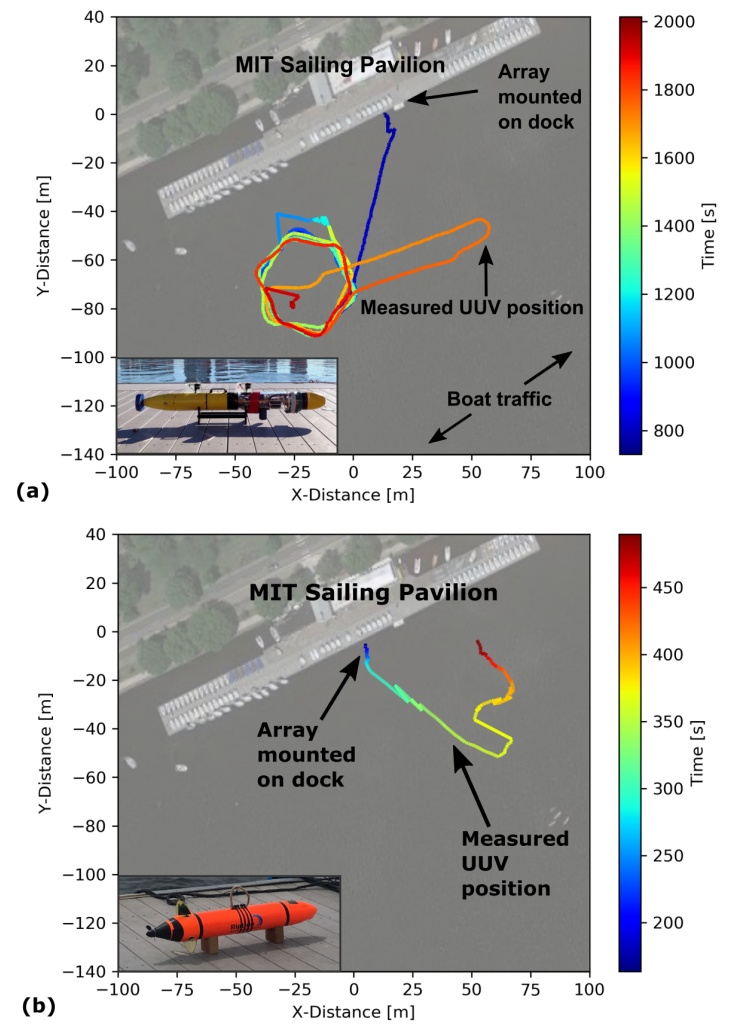

Figure 3-1: During field experiments with the (a) Sandshark and (b) Riptide UUVs, acoustic data was collected from the MIT Sailing Pavilion in the Charles River. The vehicle position over time, plotted here, was logged by the IMU-based navigation system.

\subsubsection{Identifying UUV Acoustic Features}

The acoustic signatures of the two micro-UUVs were separately measured on an off-board hydrophone array (HTI-96-MIN, $-165 \mathrm{~dB}$ re. $1 \mathrm{~V} / \mu \mathrm{Pa}$ hydrophones, which were also used in the subsequent experiments) in a quiet, controlled environment at a MIT campus swimming pool. In order to derive the acoustic signature of the UUV, the power spectral density (PSD) was estimated from time series acoustic data collected on a single hydrophone element in the line array using Welch's method [124] with a Hann window, segments of $1 \mathrm{~s}$ (and $0.5 \mathrm{~s}$ for the field tests), and $50 \%$ overlap. During the experiment, the vehicle was secured in place and the propeller rotated at different speeds for 30 seconds. Following the speed data collection, 
the thruster was off, and the fins moved a prescribed five degrees each five seconds. The sampling rate of the array was $60,060 \mathrm{~Hz}$. The measured motor tones that are above the Nyquist frequency have the subscript, "aliased". For example the true $2 f_{\mathrm{PWM}}$ is measured as $2 f_{\mathrm{PWM} \text {,aliased. }}$. This process was repeated for both the Sandshark and Riptide UUVs. At the MIT Towing Tank facility, the UUV thruster isolation experiment was performed in a tank, where the system was bottom-mounted and acoustic measurements were taken off-board.

For each of these experiments, DEMON measurements were calculated using a 10$25 \mathrm{kHz}$ fifth-order Butterworth bandpass filter to capture the propeller noise. The amplitude envelope was determined by the magnitude of the Hilbert transform applied to the bandpass filtered signal. The amplitude envelope was downsampled to $1000 \mathrm{~Hz}$, using the root mean square (RMS) method described in Chung et al [44]. Finally, the DEMON spectrum was calculated from the FFT of the amplitude envelope.

\subsubsection{Speed and Propeller Rotation Measurement Method}

The relationship between strong acoustic tones emitted by the propulsion system and the rotations per minute of the thruster was determined experimentally. When the turn-perknot ratio was known, as in the case of the Sandshark, vehicle speed was determined from the acoustic measurements. We verified that there is a strong tone at the $f_{\mathrm{PWM}}$ of the motor, which acts as a carrier to sidebands of spacing $f_{\mathrm{m}}$, equal to the rotation frequency of the motor, $f_{\mathrm{s}}$, multiplied by the number of permanent magnetic poles in the motor, $p$ :

$$
f_{\mathrm{m}}=f_{\mathrm{s}} p
$$

The resulting sidebands center around $f_{\mathrm{PWM}}: f_{\mathrm{PWM}} \pm k f_{\mathrm{m}}$ and $f_{\mathrm{PWM}} \pm n f_{\mathrm{s}}$, where $k$ and $n$ are the respective harmonic orders. The first order of $k$ dominates, $f_{\mathrm{PWM}} \pm f_{\mathrm{m}}$. Therefore, the measured sidebands can be used to predict $p$ for classification and $f_{\mathrm{s}}$ for speed estimation. With DEMON analysis, where $f_{\mathrm{m}}$ is the dominant tone and $f_{\mathrm{s}}$ is also present, these values can be verified. In practice, the sideband values were measured through peak-finding and symmetry, and validated by the DEMON measurement results. 


\subsubsection{Demonstration of Speed Estimation in Field Experi- ments}

With knowledge of the motor acoustic features identified in the pool and tank, field experiments were completed in the Charles River of Boston, Massachusetts (approximately $6 \mathrm{~m}$ deep) to evaluate motor noise as a remote speed estimation method. Two experiments were performed: the Sandshark UUV test on October 31, 2016 and the Riptide UUV test on August 29, 2019. In both experiments, the hydrophone array was mounted to the MIT Sailing Pavilion dock and the vehicle performed an autonomous mission, programmed with MOOS-IvP autonomy software [42]. A birds-eye view of both experiments is in Figure 3-1. For the Sandshark test, the river current was insignificant and therefore not accounted for in estimating vehicle speed. Taken from the vehicle's INS, the average vehicle depth and speed were respectively $1.90 \pm 0.26 \mathrm{~m}$ and $1.354 \pm 0.098 \mathrm{~m} / \mathrm{s}$. In comparison, for the Riptide test, the robot's average depth and speed were: $-0.123 \pm 0.13 \mathrm{~m}$ (at the surface) and $0.835 \pm 0.452 \mathrm{~m} / \mathrm{s}$. The array sampling rates for the Sandshark and Riptide experiments were: $37,500 \mathrm{~Hz}$ and $60,060 \mathrm{~Hz}$. The sideband spacing measurement error is reported as half of the peak width at half the height of the peak's prominence.

\subsection{Results}

\subsubsection{Sandshark UUV Acoustic Signature}

To identify acoustic features of the Sandshark UUV, vehicle noise data was collected at different speeds (400 rpm, $800 \mathrm{rpm}$, and $1000 \mathrm{rpm}$ ) for 30 second time intervals, followed by isolated fin movements. The spectrogram of the entire experiment in Figure 3-2(a) highlights two distinct tones which are then magnified in Figure 3-2(b)-(c). The tones, $19,201 \mathrm{~Hz}$ and $21,650 \mathrm{~Hz}$, are dominant and constant over all speeds. Based off of previous work in characterizing motor noise $[63,64,66-70]$ and the motor specifications of the vehicle, we infer that these tones correspond to $f_{\mathrm{PWM}}$ and its multiple, $2 f_{\mathrm{PWM}}$,aliased. The observed tone is close to the manufacturer provided range for the $f_{\mathrm{PWM}}$ of this specific motor: $15-19 \mathrm{kHz}$. In addition, the measured signal, $21,650 \mathrm{~Hz}$ is a $0.04 \%$ percent error of the expected value for

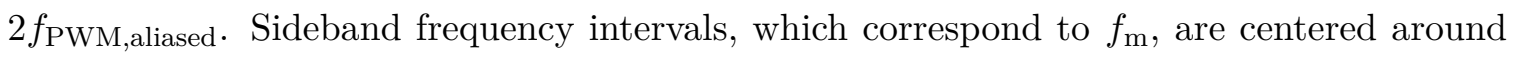
the carrier $f_{\mathrm{PWM}}$, (Figure 3-2(b)) and its second multiple, $2 f_{\mathrm{PWM} \text {,aliased }}$ (Figure 3-2(c)). To 
illustrate these observed acoustic features at a single speed, the PSD for $400 \mathrm{rpm}$, is centered at $19,205 \mathrm{~Hz}$ in Figure 3-3(a) and at 21,650 Hz in Figure 3-3(b) (the respective center tones for this speed specifically). Grey lines drawn at the expected sideband values of $f_{\mathrm{m}}$ or $p f_{\mathrm{s}}$. Additional sidebands are observed at $f_{\mathrm{s}}$, as labelled in Figure 3-3(a). The DEMON spectrum for this speed in Figure 3-3(c), agrees with the motor noise: there is a strong tone at $f_{\mathrm{m}}$ and its second multiple. Following the vehicle speed acoustic measurements, the sound of fin movement was characterized. There are two main fin noise tones that are observed at 20,003 Hz (labelled in Figure $3-2\left(\right.$ a)) and $20,053 \mathrm{~Hz}$, which we attribute to $f_{\mathrm{PWM}}$ and $2 f_{\mathrm{PWM} \text {,aliased }}$.

\subsubsection{Riptide UUV and T200 Thruster Acoustic Signature}

For the Riptide UUV noise characterization experiment, the vehicle was programmed to increase its desired speed in 30 second time intervals from $1 \mathrm{~m} / \mathrm{s}$ to $5 \mathrm{~m} / \mathrm{s}$. As shown in the spectrogram in Figure 3-4(a) across all five speeds, a tone is observed at 17,898 Hz, which is within a $0.23 \%$ percent error of the manufacturer specified $f_{\mathrm{PWM}}$. Sidebands on this center tone correspond to $f_{\mathrm{m}}$, which is verified by performing DEMON analysis in Figure 34(b). In addition, there is a strong and consistent tone at $24,270 \mathrm{~Hz}$ that we attribute to be $2 f_{\mathrm{PWM}}$,aliased. For the desired speed of $1 \mathrm{~m} / \mathrm{s}$, the PSD is centered at $f_{\mathrm{PWM}}$ in Figure 3 $3(\mathrm{~d})$ and at $2 f_{\mathrm{PWM}}$,aliased in Figure 3-3(e). Grey lines are drawn to demonstrate how the sidebands align with $f_{\mathrm{m}}$ or $p f_{\mathrm{s}}$. The DEMON spectrum of this speed in Figure 3-3(f) supports the observation that $f_{\mathrm{m}}$ is a dominant tone. The additional sideband intervals that are observed in (d)-(f) correspond to the fundamental motor rotation frequency, $f_{\mathrm{s}}$.

To support the conclusions on the sources of acoustic noise from the BLDC motor in the two micro-UUVs, a motor isolation experiment was performed. Figure 3-5 is a spectrogram of this experiment, where the propellor rotational speed increased in time intervals of 30 seconds. As predicted, the motor emits two tones at $f_{\mathrm{PWM}}$ and $2 f_{\mathrm{PWM}}$, as well as sideband spacings which align with $f_{\mathrm{m}}$. For all speeds, a steady tone at $24,008 \mathrm{~Hz}$ is observed which corresponds to the manufacturer specified $f_{\mathrm{PWM}}$ of the motor with $0.033 \%$ percent error. In addition, the measured value of $2 f_{\mathrm{PWM}}$,aliased is $12,044 \mathrm{~Hz}$, exactly matching the expected value. 

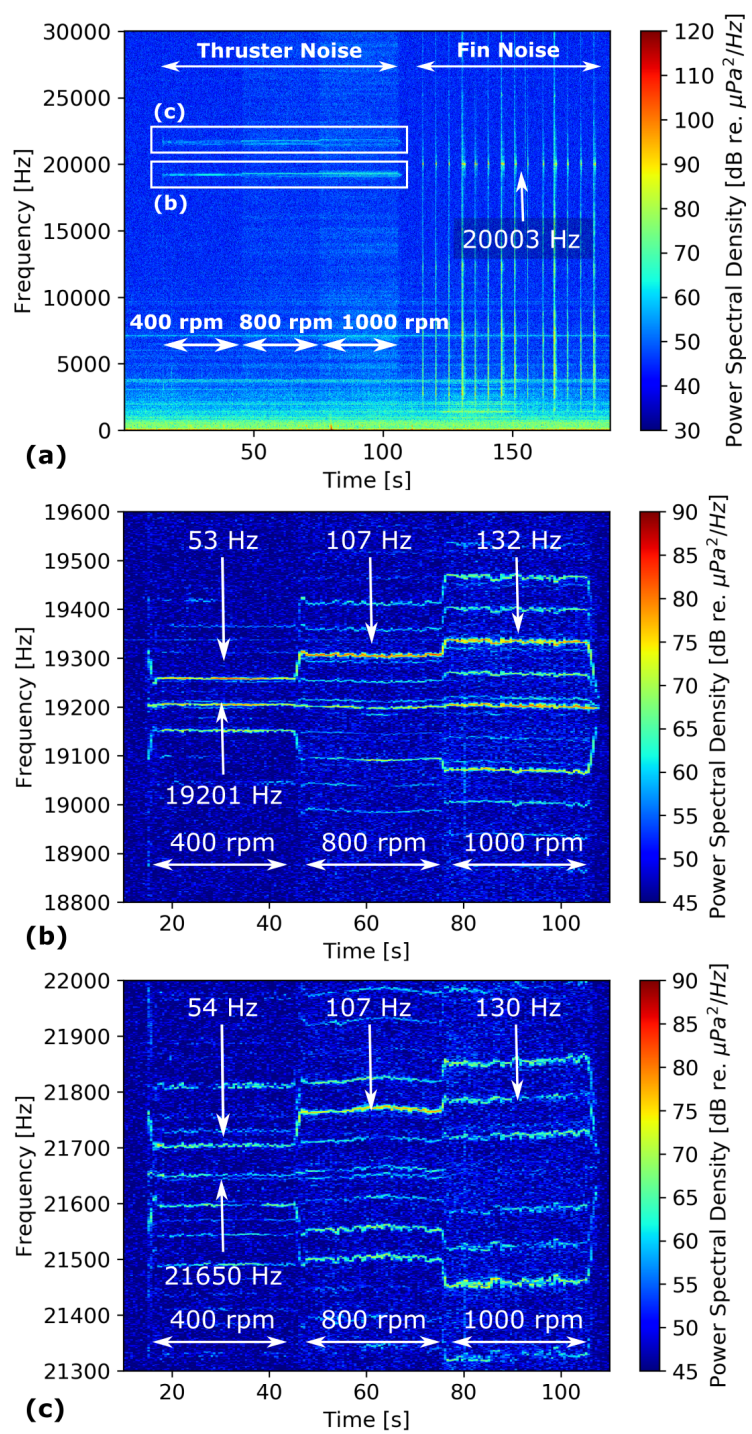

Figure 3-2: (a) The radiated noise from a Sandshark UUV was characterized at different speeds, followed by isolated fin movements. Three features are highlighted: (b) a constant tone at $19,201 \mathrm{~Hz}$, which matches $f_{\mathrm{PWM}}$ and sidebands that increase with speed; (c) a tone at $2 f_{\mathrm{PWM} \text {,aliased }}$

\subsubsection{Field Measurements of UUV Acoustic Features}

With insight into the acoustic spectral features of micro-UUVs, this information is used to demonstrate that the speed of the Riptide and Sandshark vehicles can be passively identified in the field under realistic conditions. In the spectrogram of the Sandshark experiment in Figure 3-6(a), there are two prominent features that we match to the pool measurements of the UUV after accounting for aliasing: a tone at $18,270 \mathrm{~Hz}$ corresponds to $f_{\mathrm{PWM} \text {,aliased }}$ of the thruster with sidebands spaced by $142 \mathrm{~Hz}\left(f_{\mathrm{m}}\right)$ and a tone at $17,504 \mathrm{~Hz}$ corresponds to 

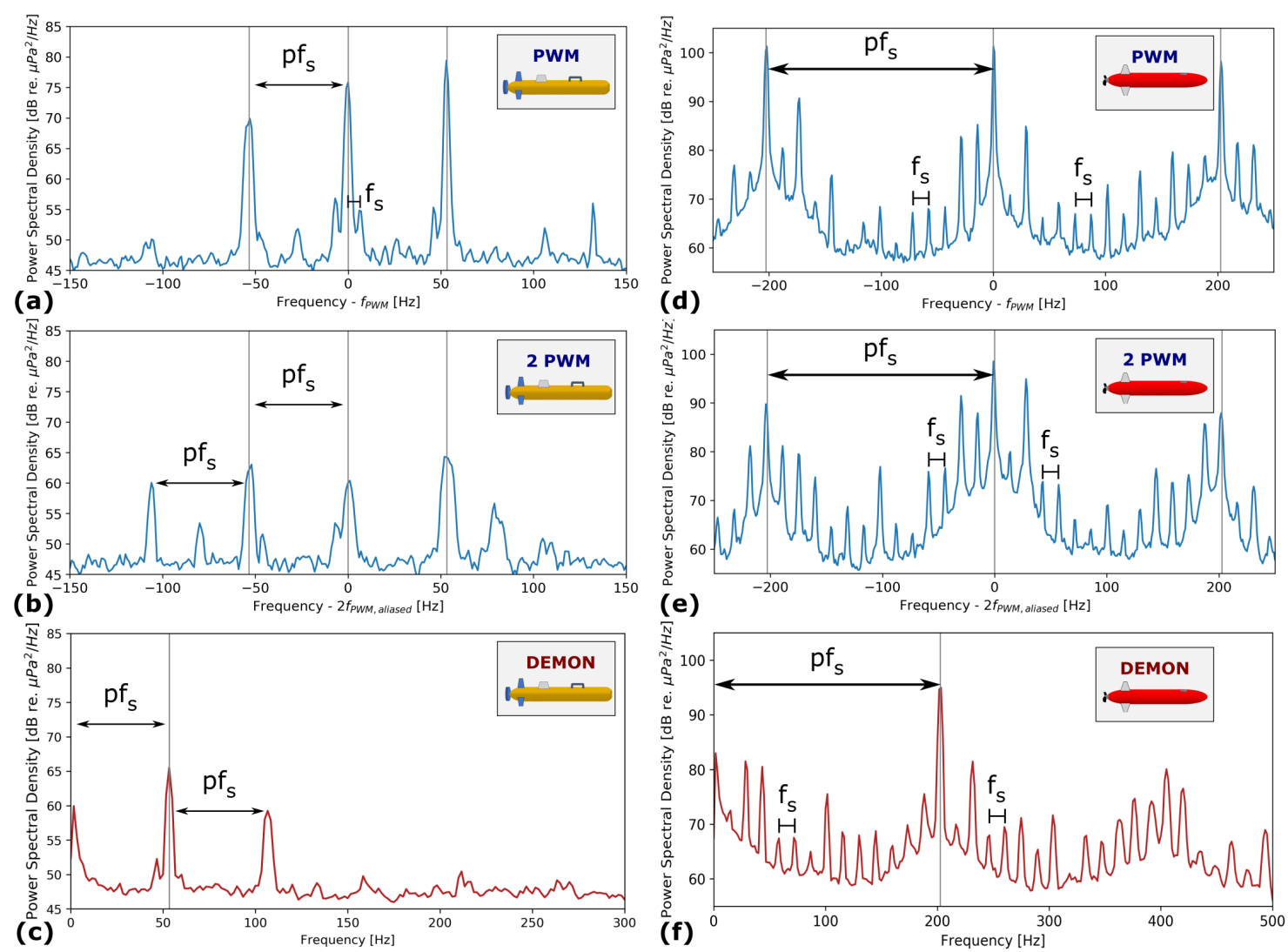

Figure 3-3: The radiated noise of the Sandshark at $400 \mathrm{rpm}$ and the Riptide at approximately $870 \mathrm{rpm}(1 \mathrm{~m} / \mathrm{s}$ desired speed) are characterized in plots (a)-(c) and (d)-(f) respectively. The PSD plots are centered at $f_{\mathrm{PWM}}$ and $2 f_{\mathrm{PWM}, \text { aliased }}$ which are: (a) $19,205 \mathrm{~Hz}$ (b) $21,650 \mathrm{~Hz}$ (d) $17,884 \mathrm{~Hz}$, and (e) $24,292 \mathrm{~Hz}$. The DEMON spectrum is plotted in (c) and (f). Grey lines indicate $p f_{\mathrm{s}}$ and the spacing of $f_{\mathrm{s}}$ is annotated.

the $f_{\mathrm{PWM}, a l i a s e d}$ of the fin servomotors. As illustrated in Figure 3-6 (b), the navigation data also supports this conclusion: when the vehicle is underway, the thruster is on and tonal features $\left(f_{\mathrm{PWM}, \text { aliased }}\right.$ and $\left.f_{\mathrm{m}}\right)$ are present. The consistent SNR of the radiated UUV noise while the robot loitered in a circle (about $15 \mathrm{~dB}$ and $45 \mathrm{~dB}$ above the background noise for the thruster and fin noise) indicates that the motor noise may be omni-directional. Also, when a boat passes, such as the time after $1600 \mathrm{~s}$, the fin motor noise is still observable (about $10 \mathrm{~dB}$ above the background noise).

Acoustic features observed in the Riptide field experiment also aligned with characteristics identified in the pool test: a strong tone at $f_{\mathrm{PWM}}$ and $2 f_{\mathrm{PWM}}$, and sidebands of spacing $f_{\mathrm{m}}$. As noted in the spectrogram in Figure 3-7 (a), there is a consistent tone at 17,890 Hz when the vehicle is underway, which matches the measured $f_{\mathrm{PWM}}$ from the pool $(0.045 \%$ 


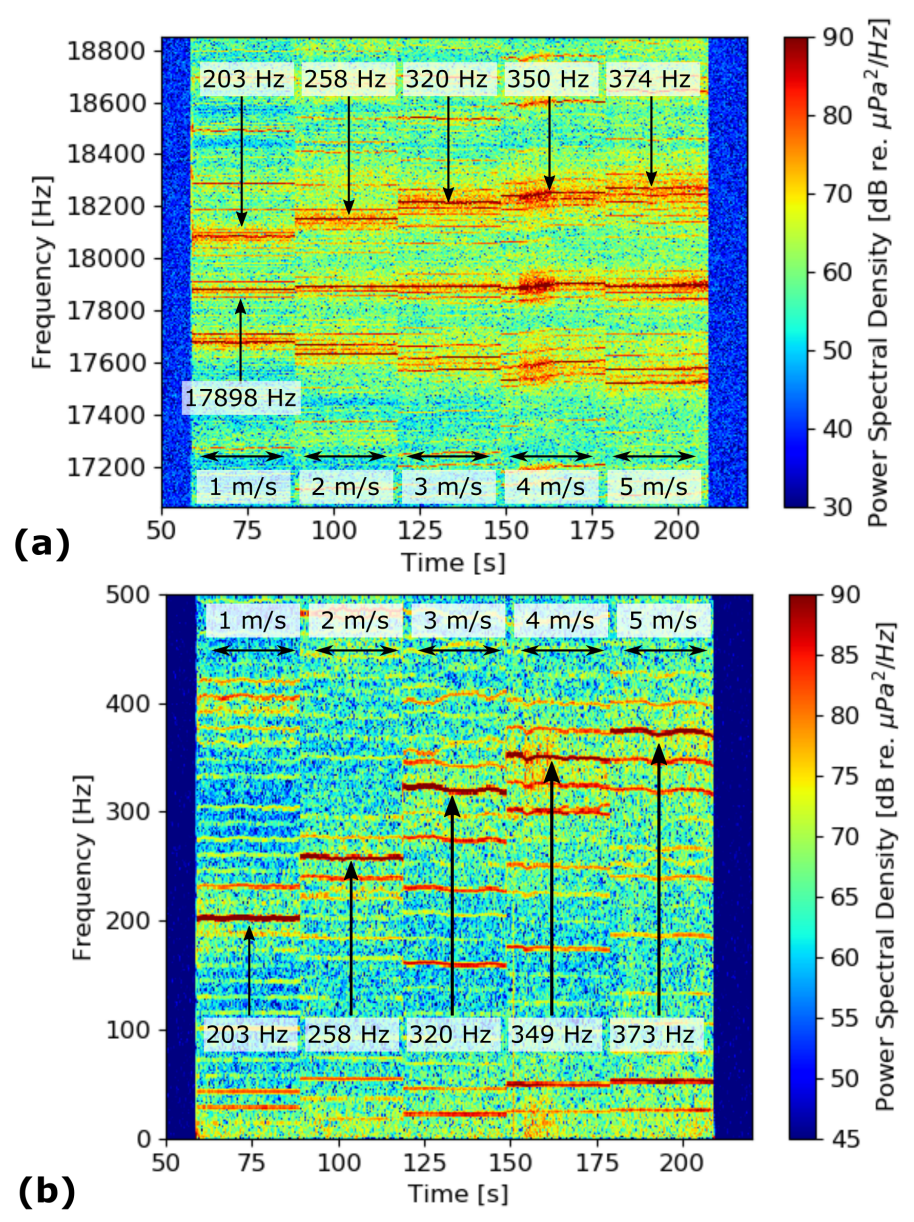

Figure 3-4: (a) A spectrogram of the Riptide UUV noise characterization test is centered at $f_{\mathrm{PWM}}$. (b) The DEMON spectrum is plotted below. Sideband spacings in (a) correspond to the tones in the (b).

percent error). A second tone at $24,264 \mathrm{~Hz}$ aligns with the expected value of $2 f_{\mathrm{PWM}}$,aliased. Sideband spacings of $212 \mathrm{~Hz}$, corresponding to $f_{\mathrm{m}}$ are centered on the carrier frequency when the desired speed is $1 \mathrm{~m} / \mathrm{s}$. The sideband spacing increases to $251 \mathrm{~Hz}$ when the vehicle changes its desired speed to $1.5 \mathrm{~m} / \mathrm{s}$. Figure $3-7$ (c) shows the desired and actual speed of the vehicle from the INS. Dashed black lines illustrate how the vehicle speed aligns with the radiated acoustic noise. The depth of the vehicle was not included in the navigation plot because the vehicle was on the surface for this exercise. To show how the high frequency motor noise compares to the propeller cavitation noise, the DEMON spectrum is plotted in Figure 3-7 (b). The annotated tones of $212 \mathrm{~Hz}, 424 \mathrm{~Hz}$, and $251 \mathrm{~Hz}$ correspond to $f_{\mathrm{m}}$, matching the sideband spacings in Figure 3-7 (a). These features are more challenging to observe in the DEMON spectrum (about $15 \mathrm{~dB}$ above the background noise) and at times, 


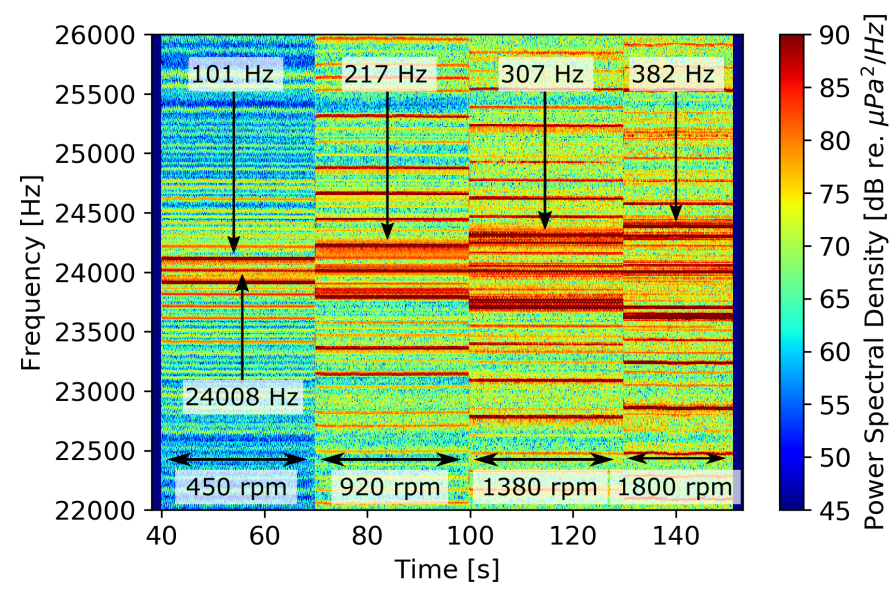

Figure 3-5: The spectrogram of the T200 motor isolation experiment is centered at $24,008 \mathrm{~Hz}\left(f_{\mathrm{PWM}}\right)$. As the speed increases, sidebands centered around the $f_{\mathrm{PWM}}$ carrier increase their interval spacing.

such as between $340 \mathrm{~s}$ and $360 \mathrm{~s}$, not observable. The change in SNR could be explained by the characteristic radiation pattern of the propeller noise. For example, the vehicle starts by traveling at a constant heading, away from the dock (See Figure 3-1) and the tonal features in the DEMON spectrum fade away until the vehicle changes direction at around $360 \mathrm{~s}$. The motor noise has minimal variation and appears to be omni-directional (at about $30 \mathrm{~dB}$ above the background noise). The motor noise is also observable when a boat passes, about $10 \mathrm{~dB}$ above the background noise, while the DEMON spectrum is dominated by the interfering boat's cavitation noise.

\subsubsection{Derivation and Field Demonstration of Speed and Pro- peller Rotation Estimation Method}

To quantify the relationship between acoustic noise and vehicle speed, three different acoustic characterization experiments are compared - the Sandshark UUV, Riptide UUV, and T200 thruster - which involved BLDC motors of different specifications. The $f_{\mathrm{PWM}}$ and number of permanent magnetic poles, $p$, varies for the motor inside each of these systems. Therefore, as described in this section, we were able to verify the linear relationship of Eq. (3.1) between the rotational speed of the motor, $f_{\mathrm{s}}$, and the sidebands with frequency intervals of $f_{m}$, centered on $f_{\mathrm{PWM}}$. Also in this section, we show that the acoustic field measurements of an underway UUV can be used to extrapolate the vehicle speed by measuring 


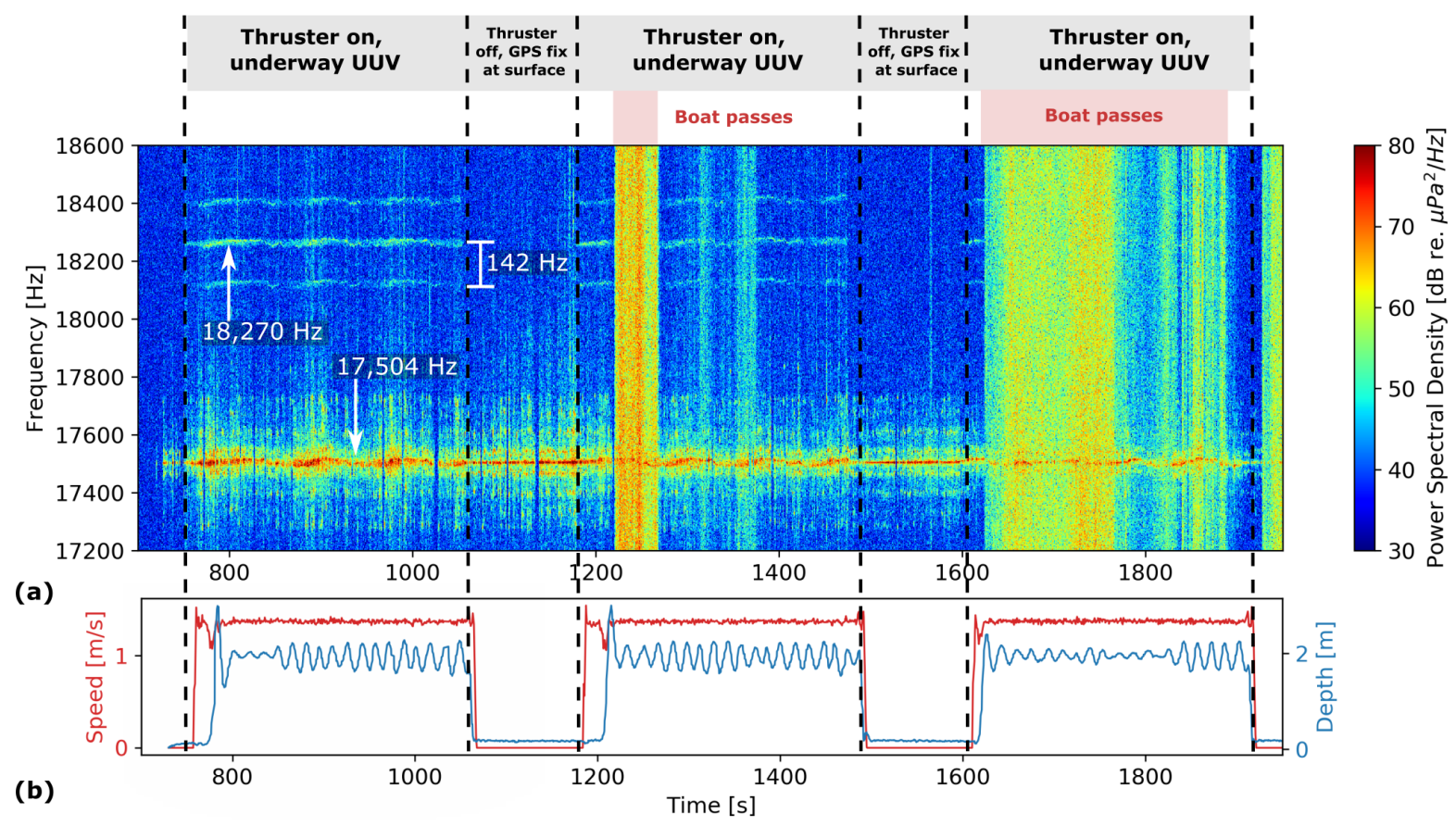

Figure 3-6: (a) The spectrogram of acoustic data from the Sandshark noise experiment in the Charles River is compared to (b) the vehicle navigation data. The tone at $18,270 \mathrm{~Hz}$ with sideband spacing at $142 \mathrm{~Hz}$ and the tone at $17,504 \mathrm{~Hz}$ correlate to the PWM switching frequencies of the thruster motor and fin servomotors. The former tone is present when the vehicle is underway.

the sidebands.

For the T200 thruster test, the measured sideband spacings, $f_{m}$, are plotted against the motor's corresponding speeds in Figure 3-8(a). Applying a linear fit, the estimated pole number from the acoustic measurements was 13.1 (the true number of poles is 14). At the time of the Riptide UUV noise experiment, we did not have access to the true $f_{\mathrm{s}}$ mapping to the UUV desired speed that was used for programming the vehicle. Therefore, in Figure 3-8 (b), the measured sideband spacings from the pool experiment are plotted over the desired speed of the robot and a second order fit is applied. The sideband spacing values of $212 \mathrm{~Hz}$ and $251 \mathrm{~Hz}$ from the field are also plotted to show how well this relationship could predict the speed of the vehicle. The estimated speeds were $1.15 \mathrm{~m} / \mathrm{s}$ and $1.69 \mathrm{~m} / \mathrm{s}$ which resulted in a percent error of $15.4 \%$ and $12.8 \%$ respectively, compared to the true desired speed. The pool experiment results of the Sandshark UUV sideband spacing values, $f_{m}$, at three different speeds are plotted in Figure 3-8(c). Given the number of poles in the Sandshark thruster motor ( 8 poles), the predicted values of $f_{m}$ from Eq. (3.1) were plotted. 
The estimated pole number from the acoustic measurements is 7.96. To demonstrate that this relationship can predict the speed of an underway vehicle with a known turn-per-knot ratio in negligible currents, we consider the period of 770 s to $1035 \mathrm{~s}$ in the Sandshark field experiment. The vehicle speed for this time segment, reported by the INS of the robot was $1.36 \pm 0.04 \mathrm{~m} / \mathrm{s}$ or $1079 \pm 29 \mathrm{rpm}$ (drawn in Figure $3-8(\mathrm{c})$ ). The measured sideband spacing for this segment was $142 \pm 6 \mathrm{~Hz}$. From Eq. (3.1), with the known pole number of 8 , the expected value of the vehicle speed is $1.34 \pm 0.08 \mathrm{~m} / \mathrm{s}$ or $1065 \pm 32 \mathrm{rpm}$, which is within the error range of the INS reported vehicle speed and yields a $1.3 \%$ error of the rpm measurement, using the INS value as truth. Therefore, with this insight into the relationship between $f_{m}$ and $f_{\mathrm{s}}$, we demonstrate that the speed of the Sandshark UUV can be estimated in the field by measuring the sidebands.

\subsection{Conclusion}

In this work, a method for passively estimating the propeller rotation $\left(f_{\mathrm{s}}\right)$ and speed of a UUV is derived and evaluated through experimentation, and compared to the DEMON algorithm. The acoustic signatures of two micro-UUVs, the Sandshark and Riptide, were measured at a range of speeds to pinpoint sources of spectral noise. To isolate noise emitting from the propulsion system, the T200 thruster was also characterized. From these experiments, we determined that the PWM switching frequency, $f_{\mathrm{PWM}}$, and its multiple, $2 f_{\mathrm{PWM}}$, of the modulated voltage signal that drives the BLDC motor in these propulsion systems are dominant sources of acoustic noise. In addition, there are sidebands of spacing, $f_{\mathrm{m}}$ (the prominent sideband value) and $f_{\mathrm{s}}$, that are centered around $f_{\mathrm{PWM}}$. We validated, through experimentation, that the sideband spacing of $f_{\mathrm{m}}$ is equal to $f_{\mathrm{s}}$ multiplied by the number of permanent magnetic poles, $p$, in the motor (Eq. (3.1)). When the sideband spacing of $f_{\mathrm{s}}$ is also present in the motor noise signature, $p$ can be determined. Furthermore, if DEMON measurements are observable, they can be used to verify these values.

The radiated noise of the UUVs was measured in the Charles River, which is a shallow water environment with dynamic and loud ambient noise from passing boats, construction, and motor vehicle traffic on bridges. The UUV propeller rotation frequency was extrapolated by measuring the associated harmonics of the $f_{\mathrm{PWM}}$ carrier. In the case of the Sandshark, where the turn-per-knot ratio is known, the vehicle speed was predicted with 
$1.3 \%$ accuracy, and within the error range of the INS reported speed. As demonstrated in the field tests, measuring the motor noise signature has several advantages over the DEMON method: simplicity (in regards to choosing an optimal passband for cavitation noise), a higher SNR, and an omni-directional radiation pattern. Furthermore, when boats passed by the UUV, the motor noise is still observable, but the DEMON spectrum is dominated by the interfering boat's propeller cavitation noise. Because the radiation pattern due to machinery vibration depends how the machinery (the motor in the case) is mounted to the hull, we predict that a large section of the UUV hull is vibrating at the motor tones we observe. This path of vibration from the motor to the water could explain the omnidirectional pattern of noise and high SNR. Understanding the origins of acoustic noise in these autonomous platforms can inform quieter UUV propulsion design to avoid interference with onboard sensors and disturbance to marine life for wildlife monitoring applications. By predicting the potential electromagnetic vibration frequencies, the mechanical system can be designed to avoid structural resonance. Vibration isolation mounts could also be installed to minimize the paths of vibration to the hull. In the future, based off of these findings, quieting techniques for BLDC motors in UUVs can be compared and assessed, particularly for mitigating $f_{\mathrm{PWM}}$.

Another important application of this work is passively tracking UUVs. A BLDC motorpowered vehicle can be detected and classified by its unique acoustic signature of high frequency harmonics of $f_{\mathrm{PWM}}$. As shown with the case of the Sandshark, extrapolating $f_{\mathrm{s}}$ from motor noise can be utilized for speed estimation, if the turn-per-knot ratios are known. A next step for this research is to incorporate target motion analysis, which will be the primary focus of our follow-on work. The extensive research done in UUV navigation, summarized in Liam et al, can be leveraged for selecting a state estimate technique [31]. For extending this work to other vehicles and environments with non-negligible currents, a combination of dynamic and kinematic models, with current velocity in the state vector, have been demonstrated with success [125-127]. Furthermore, in cases where there are multiple marine vessels present like ship traffic and UUVs, multi-source tracking could be investigated. Using the micro-UUV signatures obtained experimentally, passively tracking the motor noise under various environmental conditions can be assessed.

In conclusion, we have experimentally identified acoustic features in two micro-UUVs and a thruster in isolation which we attribute to originate from the BLDC motor in their 
respective propulsion systems. These findings have the potential to inform future designs of marine robotic platforms that also rely on off-the-shelf BLDC motors and are used for low-noise applications like tracking marine life. Lastly, these results can be used to improve target motion analysis applied to UUVs. 


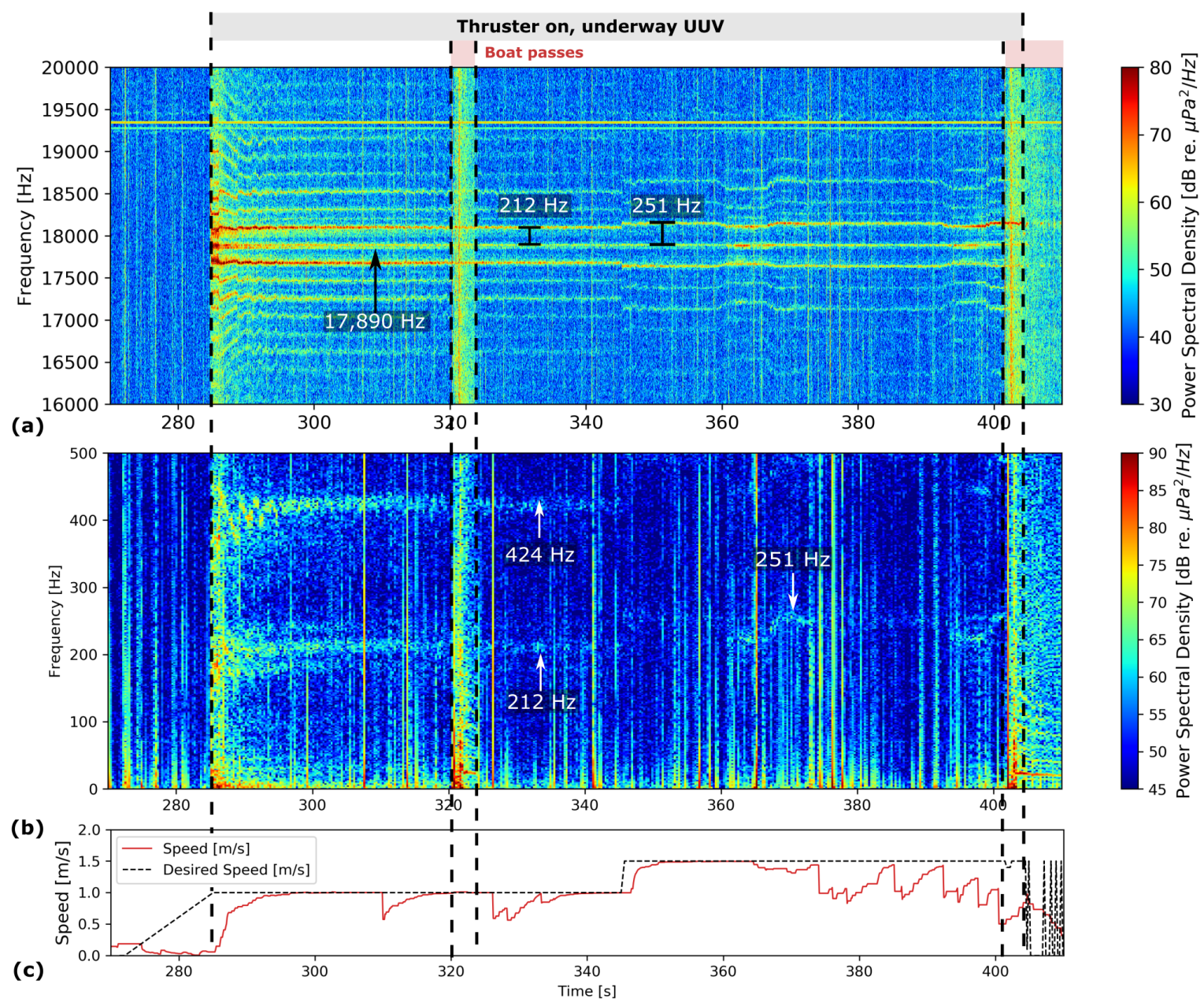

Figure 3-7: (a) The spectrogram of acoustic data from the Riptide noise experiment in the Charles River is compared to (b) the DEMON spectrum, and (c) the vehicle navigation data from the INS of desired and actual speed. In (a), the constant tone at $17,890 \mathrm{~Hz}$ corresponds to $f_{\mathrm{PWM}}$ with sideband spacings of $f_{\mathrm{m}}$ at $212 \mathrm{~Hz}$, which increase to $251 \mathrm{~Hz}$ with a desired speed change. The values of $f_{\mathrm{m}}$ are also present in (b). 

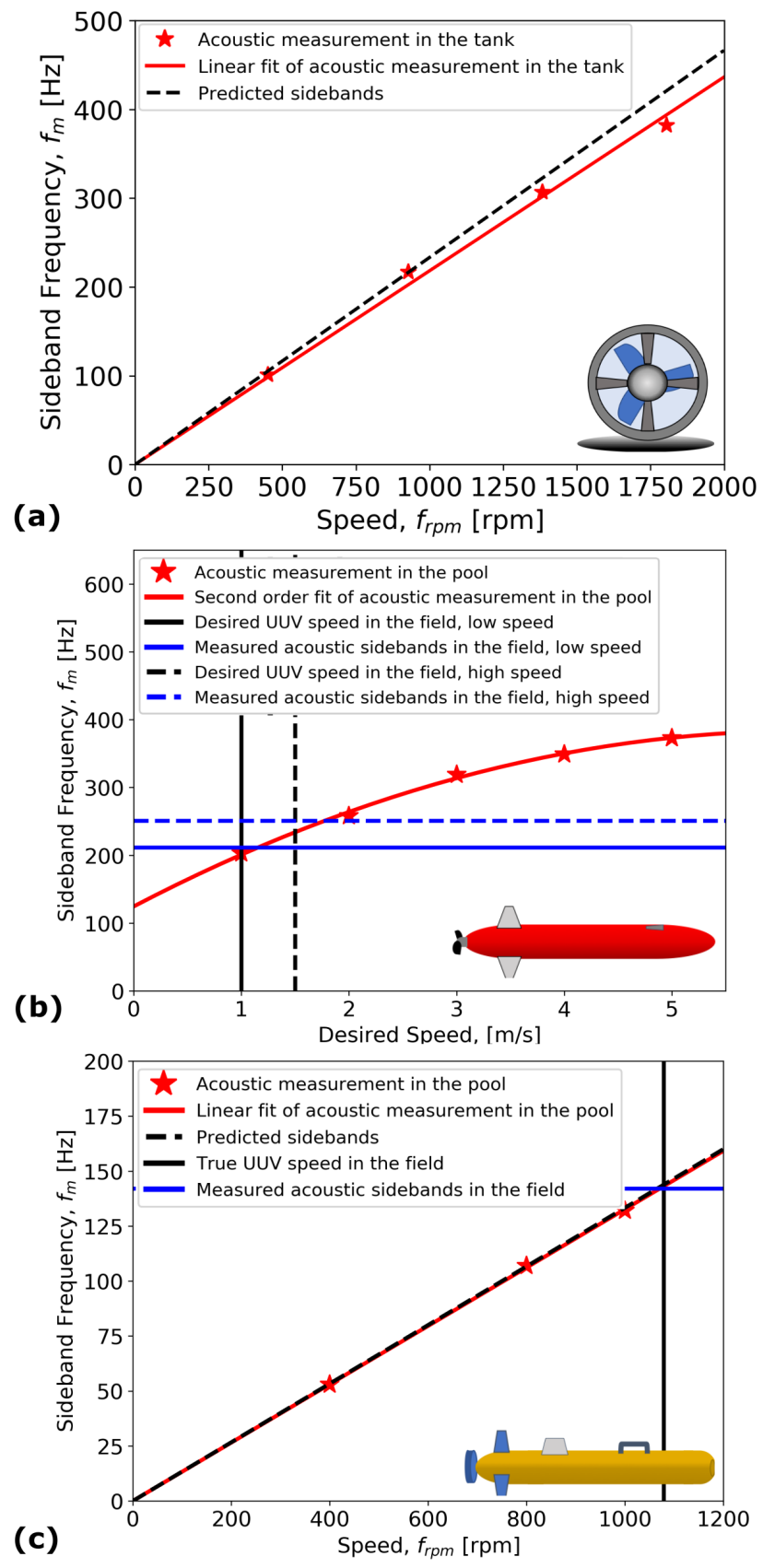

Figure 3-8: The acoustic measurements of the sideband frequency intervals, $f_{m}$, are compared to the corresponding motor speeds, $f_{\mathrm{s}}$, for the (a) T200 thruster and (c) Sandshark UUV, and the desired speed for (b) the Riptide vehicle. The predicted sideband spacings are in dashed black. In (b) and (c) lines are drawn to show measured sideband spacings versus the true vehicle speed in the field experiments. 


\section{Chapter 4}

\section{Passive Acoustic Detection and Tracking of a UUV from High-frequency Motor Noise}

\subsection{Introduction}

Passive acoustic detection, classification, localization, and tracking (DCLT) of marine life and vessels is an essential technology for ecological assessment and maritime surveillance. For defense applications, identifying small vessels - divers, boats, and robotic platforms - by their unique acoustic signature of radiating self-noise is necessary when other measurement methods fall short. For example, detecting and ultimately, preventing a diver from delivering an explosive device to a domestic harbor is a challenging problem. Currently, active sonar systems are deployed for harbor surveillance of divers [128]. Passive sonar, however, has several advantages over active sonar for long-duration monitoring in shallow-water: minimal disturbance to marine life, less multi-path, fewer false alarms, and lower power. Passive sonar has been demonstrated to detect the breathing patterns of divers and their equipment [115,128-130]. Another application of passive sonar in national security is classifying which vessels are authorized to navigate in marine protected areas and monitoring harbor traffic for maritime law enforcement. Small boats are difficult to detect above water with radar, [46] and optical methods are limited by weather for visibility $[113,131]$. In addition to filling the 
gaps of these remote sensing methods, passive sonar is inherently covert, which is essential in defense applications.

A new requirement for maritime surveillance systems is the capability to detect and track unmanned underwater vehicles (UUVs) [24-26]. UUVs are capable of autonomously and adaptively tracking moving noise sources for anti-submarine warfare (ASW). These autonomous platforms can be equipped to perform DCLT with passive sonar [94,132], and with active sonar (a bistatic or multistatic approach) [133-136]. Therefore, maintaining situational awareness of these robotic platforms by detecting their radiating acoustic noise signature with passive sonar is imperative. Another need for passive acoustic tracking of UUVs is to prevent drug-trafficking. These platforms have many attractive features for smuggling: they are low-profile, inexpensive, and unmanned (no personnel can be captured if the vehicle is seized). Drug-trafficking organizations from South America have already developed self-propelled semi-submersible vessels to smuggle drugs into the United States [137]. The U.S. Foreign Military Studies Office data reports that $80 \%$ of drugs smuggled into the U.S. in 2012 were transported through maritime routes, $30 \%$ of which were smuggled on narco subs [138]. Finally, there is growing concern that UUVs are armed and navigating in harbors and restricted waterways [26].

These autonomous marine platforms pose a difficult challenge for passive acoustic monitoring systems because of their low-noise acoustic profile. As reported in Holmes et al. [119], UUV acoustic signatures from $100 \mathrm{~Hz}$ to $10 \mathrm{kHz}$ - of the REMUS, Odyssey, Autosub, and Ocean Explorer class UUVs at 3 to 5 knots - have $1 / 3$ octave band source levels at 80 $150 \mathrm{~dB}$ re $\mu \mathrm{Pa}$ at $1 \mathrm{~m}$. Detecting UUVs in close proximity to ship and boat traffic, which emit loud broadband noise, is exceedingly difficult, especially in the low frequency regime. For example, Barlett et al. published source levels of small boats at $125-150 \mathrm{~dB} / \mathrm{Hz}$ at $1 \mathrm{~m}$ from $200 \mathrm{~Hz}$ to $1200 \mathrm{~Hz}$ and Erbe et al. measured the acoustic noise of rigid-hulled inflatable boats to be $80-160 \mathrm{~dB}$ re $1 \mu \mathrm{Pa}^{2}$ at $1 \mathrm{~m}$ from $100 \mathrm{~Hz}$ to $10 \mathrm{kHz}[139,140]$.

The first step in effectively tracking these quiet platforms with passive acoustics is to understand the major sources of noise generated by the vehicle, particularly machinery and cavitation noise. Acoustic noise radiates from all vessels with rotating and reciprocating mechanisms used for propulsion and stability. The generated machinery noise can transfer to the surrounding water through the hull, which could be picked up by a distant hydrophone. Another major source of noise originates from propeller cavitation. Broadband cavitation 
noise contains "propeller beats" where the cavitation noise is amplitude-modulated at the rotation rate of the propeller, or at the shaft rotation frequency multiplied by the number of propeller blades [1]. The process for extracting these features is the detection of envelope modulation on noise (DEMON) algorithm. This process has been demonstrated successfully on small boats $[45,46]$ and ships [44] for the purpose of small vessel DCLT in ports and harbors.

However, using cavitation noise for passively tracking UUVs has several limitations. The vehicle creates minimal cavitation in comparison to ships and boats. In the ambient noise of harbors, which includes the sounds of surface ships, marine mammals, trains and motor vehicles on nearby bridges, and pile driving and other construction noise, propeller beats and blade lines of the vehicle are challenging to measure. As demonstrated in UUV field experiments by Railey et al., the DEMON spectrum is dominated by the interfering cavitation noise of boats [48]. Because of the limitations in using cavitation noise for target motion analysis (TMA), we focus on sources of machinery noise in these platforms.

Compared to other vessels, characterizing the acoustic features of machinery noise inside UUVs for the purpose of DCLT is under-examined. The UUV system has two modules of notable size and power which have the potential to create unwanted machinery noise: fin actuator motors for stability and a motor for propulsion. Prior work in understanding and mitigating acoustic noise in UUVs - specifically due to motors - is focused on the lower frequency regime of $10 \mathrm{~Hz}-10 \mathrm{kHz}$. For example, Cuschieri et al. investigated vibration transmission paths less than $1 \mathrm{kHz}$ due to the onboard motors (one motor for propulsion and four servomotors for control) [141]. Zimmerman et al. demonstrated how modifying the current waveform in the thruster brushless DC motor from trapezoidal to sinusoidal drive successfully decreased the overall noise of the vehicle in the frequency band of $10 \mathrm{~Hz}$ $10 \mathrm{kHz}$ [142]. In Holmes et al, an overview of self-noise levels of UUVs from $10 \mathrm{~Hz}-10 \mathrm{kHz}$, the authors point out that fluctuations in the electromagnetic field in the gap between the rotor and stator inside the propulsion system motor can lead to vibration [119]. In terms of tracking a UUV from its self-noise, Gebbie et al. demonstrated that a $1065 \mathrm{~Hz}$ tone, assumed to originate from the propulsion system, could be used to track the bearing of the vehicle over time [120]. Earlier research in characterizing and tracking a UUV from its selfnoise has been focused on the lower frequency regime (below $10 \mathrm{kHz}$ ), in quiet environments, and with bearing-only measurements. 
In our recent work, we demonstrated that the dominant source of high-frequency noise radiating from UUVs are the brushless DC (BLDC) motors inside the fin and propulsion systems [48]. This type of motor is extensively used in robotics because of its desirable features: low-cost, high-efficiency, high-speed, long life-span, and high torque-to-inertia ratio $[36,143]$. The BLDC motor can be found in the REMUS-100/600/6000, Sentry, Doradoclass, Iver2, [47]; Riptide [48], and Autosub [49], in addition to the Sandshark UUV [48] used in this study. This popular motor type is a permanent magnet AC (PMAC) synchronous motor with a trapezoidal back-EMF waveform. Inside the motor, permanent magnets are on the rotor and 3-phase armature windings on the stator. The concentrated stator windings result in the trapezoidal back-EMF. These motors are electronically commutated with a quasi-square wave that is pulse-width modulated (PWM) to control motor speed. Acoustic noise in BLDC motors, and PMAC motors generally, is influenced by the tangential (cogging torque and torque ripple) and radial electromagnetic forces inside the motor, as well as the mechanical resonances of the whole system $[52,69]$.

Tones at harmonics of the PWM switching frequency are observed in the Sandshark UUV. Based off of the previous findings, we hypothesize that the source of these tones are due to fluctuations in the radial electromagnetic forces in the motor. The air-gap flux density distribution in PMAC motors creates radial electromagnetic forces that are a function of the slot/pole combination, current harmonics, and back-EMF harmonics. These radial forces cause deformation in the stator core, leading to vibration and noise. Researchers have determined through simulation and experimentation that vibration from the radial electromagnetic force at the PWM harmonics produces strong tones in BLDC motors (PMAC motors with trapezoidal back-EMF and six-step commutation) [64], permanent magnet synchronous motors (PMSM) (PMAC motors with sinusoidal back-EMF and sinusoidal driving scheme) [66,67], and induction motors [68-70]. In Railey et al., it was determined that the Sandshark UUV produces loud tones at the motor PWM switching frequency and its second harmonic, with sidebands at to the pole number times the shaft rotation frequency [48]. This modulation of the PWM switching frequency carrier is expected: when the motor rotates, the excitation frequency, $f_{r}$ of the radial force on each stator tooth is: $f_{r}=p f_{s}$, where $p$ is the permanent magnet pole number and $f_{s}$ is the shaft rotation frequency $[63,64]$. Zeze et al. experimentally validated this phenomena in PMSMs: the vibration mode about the PWM carrier frequency due to the radial forces is dependent on pole number [66]. The 
trade-offs for techniques that can reduce tones at the PWM harmonics are outlined in Lo et al [69]. For example, increasing the switching frequency results in higher switching losses in the insulated gate bipolar transistors (IGBT) and MOSFET-controlled thyristor [69]. Tracking the UUV from the tones at the PWM switching frequency harmonics has several advantages. The tones are loud, high frequency, and information rich. The Doppler shift of high frequency tones from a slow-moving target are observable, and the motor acoustic signature provides the additional measurement of RPM. The accuracy of determining the true target position from fusing noisy acoustic measurements depends on the observability of the vehicle state from the available measurements, as well as high SNR measurements.

For the UUV tracking application, we assume the robot moves at a constant-velocity. There are two main approaches for tracking a constant-velocity target with non-linear measurement equations: recursive Bayesian estimation and batch processing least-squares estimation, which are described in detail in Bar-Shalom et al. [3] and Gibbs et al [76]. In the field experiments of our work, the vehicle moved in a circular pattern. As a consequence, we have a mismatch between the vehicle's actual behavior and the constant-velocity model. To resolve the mismatch, the recursive Bayesian technique - the unscented Kalman filter- was selected since it can account for model mismatch with process noise error. The unscented Kalman filter (UKF) was selected over other nonlinear state estimation methods such as the extended Kalman filter and Particle Filter for the following reasons: the UKF possesses better stability over the extended Kalman filter [77,78]; and the UKF runs more efficiently than the Particle Filter $[79,80]$. In this work, we also leverage previous research done in UUV navigation. Navigation, which also involves estimating the state of the vehicle, differs from tracking in the sensor location: the sensors are on its own platform, not remote. Advances in UUV navigation are summarized in Paull et al. [31], Leonard et al. [28], and Kinsey et al [75].

Part of our state estimation implementation is transforming RPM measurements to the underway speed of the vehicle. We incorporate the vehicle dynamic model estimation technique, developed in Randeni et al [144], which used system identification to obtain a dynamic model of the Sandshark UUV for the purpose of developing a hydrodynamic modelaided navigation system. In order to implement this technique for the tracking application, the dynamic model estimator uses the on-board UUV navigation data from a previous experiment as inputs. Therefore, this pre-estimated model embedding approach of tracking 
can only be utilized for the case of tracking a known target vehicle. However, in this study, we also evaluate the accuracy of tracking a target with an unknown dynamic model.

Tracking the UUV from bearing, Doppler, and RPM measurements is compared to traditional TMA which only involves the measurements of bearing and range rate. The significance of adding a unique measurement is an increase in the observability of the robot position from the measurements available. There has been extensive research in passively tracking an emitting source from bearing-only measurements including, but not limited to the following topics: bearing-only TMA with highly maneuverable targets [82-85], cooperative localization $[87,88]$, optimal observer trajectory [89-94], and a comparison of polar and cartesian coordinates [95]. Observability is a limitation of relying on bearing-only measurements. If the observing platform moves at a constant velocity, the target state estimate cannot be determined because there are an infinite number of values for the state estimate yielding the same observations. Therefore, the observing platform must "outmaneuver" the target [86]. When bearing and dopplerized radiated frequencies are both available, a unique TMA solution can be determined without any maneuver of the observer platform [96]. Therefore, a unique tracking solution of the target can be realized from a stationary line array. Implementing Doppler-bearing [97-102] and Doppler-only [103, 104] TMA has been thoroughly researched. Bearing-Doppler TMA is used as a baseline case for comparing tracking the UUV from bearing-Doppler-RPM measurements.

In this work, we demonstrate that the unique acoustic signature of the motor inside the UUV propulsion system can be used to accurately track the vehicle from a stationary line array. In Section 4.2.2, we describe the field experiment set-up for evaluating bearingDoppler-RPM TMA from motor noise. We describe the steps for processing the acoustic array data in Section 4.2.3, followed by the unscented Kalman filter implementation in Section 4.2.5. We present our analysis of the field experiment acoustic measurements bearing, range rate, and RPM - in Section 4.3.1. The UKF estimate of the state of the vehicle is compared to the true vehicle state in Section 4.3.4. In this section, bearingDoppler-RPM TMA is compared to traditional bearing-Doppler TMA. The robustness of the predicted dynamic model of the vehicle to model mismatch is tested in Section 4.3.5. Finally, we summarize our results and future work, as well as a discussion of the limitations of this method, in Section 4.4. 


\subsection{Methods}

\subsubsection{Description of the Unmanned Underwater Vehicle}

The General Dynamics Mission Systems Bluefin Sandshark unmanned underwater vehicle, which is pictured in Figure 4-1 (inset), was selected for the experiment. This compact vehicle with a $0.12 \mathrm{~m}$ diameter is designed with a depth rating of $200 \mathrm{~m}$, speed range of 1-2 $\mathrm{m} / \mathrm{s}$, and endurance of 6 hours [38]. The vehicle architecture includes a standard tail section and a user customizable payload section. Inside the former, there are four BLDC motors: the thruster motor for propulsion and three fin actuators for control. The thruster is powered by a BLDC motor with 8 poles (Anaheim Automation BLWR173S-24V-2000), which uses a PWM frequency between 15 and $20 \mathrm{kHz}$. The fins are controlled by a BLDC servomotor (Maxon EC-max 16), which is driven by a PWM signal. For navigation, the vehicle depends on a MEMS Inertial Measurement Unit (IMU) for estimating vehicle roll, pitch, and heading [38]. Speed-over-ground is derived from propeller RPM and periodic GPS fixes [38].

\subsubsection{Field Test Set-up}

In order to evaluate UUV detection and tracking under realistic conditions, a 2440 seconds test was performed where the radiated acoustic noise of the Sandshark UUV was remotely measured from a stationary, dock-mounted array in the Charles River of Boston, Massachusetts on October 31, 2016. The ambient noise in the Charles River environment is comparable to a port or harbor: passing boats, construction, and motor vehicle traffic on bridges are all contributors to the background noise. Furthermore, the river is subject to multi-path effects as a shallow environment with an approximate depth of $6 \mathrm{~m}$. As shown in a birds-eye view of the experiment in Figure 4-1, the array was mounted on the dock of the MIT Sailing Pavilion. The vehicle was launched from the dock and followed waypoints in a loiter pattern, programmed by MOOS-IvP autonomy software [42]. While underway, the average vehicle speed was $1.354 \pm 0.097 \mathrm{~m} / \mathrm{s}$ and the average depth was $1.91 \pm 0.26 \mathrm{~m}$. The vehicle periodically surfaced for acquiring a GPS fix, as demonstrated in a plot of the speed and depth of the vehicle over time in Figure 4-2a.

The estimated UUV position from remote acoustic measurements is compared to the 


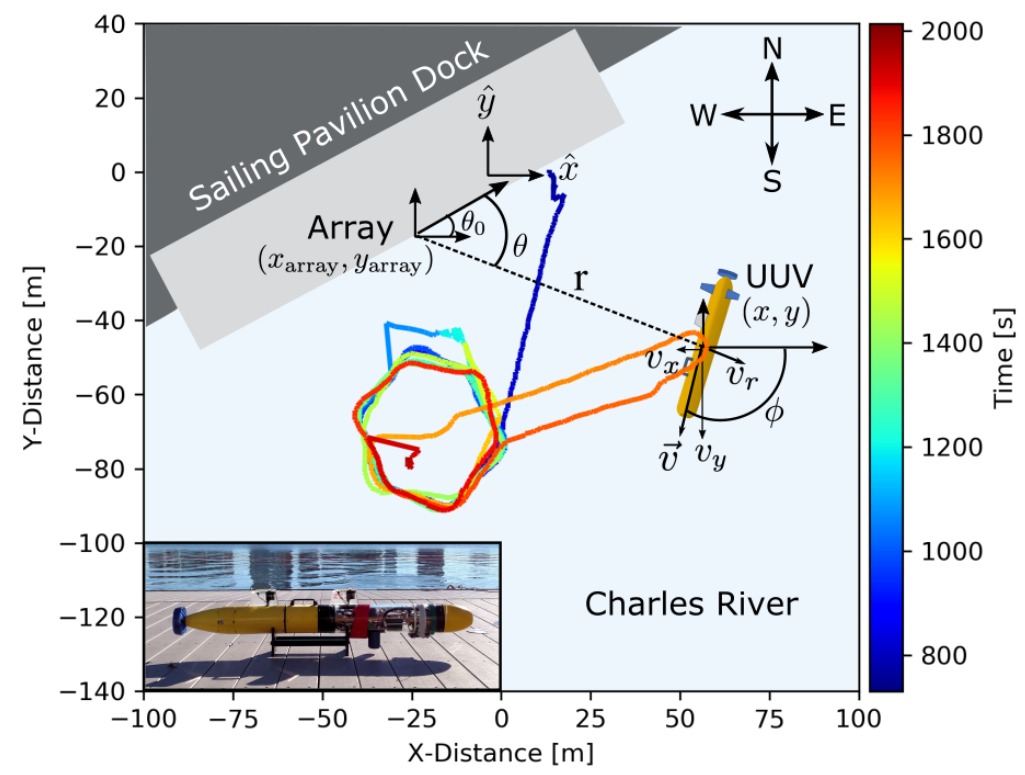

Figure 4-1: For the Charles River field experiment, the Sandshark vehicle, pictured in the corner, was deployed from the MIT sailing pavilion dock. Acoustic measurements of the radiating noise from the UUV were collected from the dock-mounted array. The vehicle IMU-based navigation solution is plotted over time, shown in color (starting in blue, ending in red). There are three relevant coordinate systems shown here. First, a local cartesian coordinate system, where the origin is dock-centered. Second, the acoustic array polar coordinate system. Third, the coordinate system fixed to the UUV.

true position of the vehicle, which, for this experiment, is the IMU-based navigation solution from the robot. One should keep in mind that the IMU-based navigation solution error accumulates over time at an approximate rate of $1 \mathrm{~km}$ after an hour of underway operation [144]. For an estimate of the expected error from the vehicle's navigation data for this experiment, the distance travelled by the vehicle between each time step is plotted in Figure 4-2b. Distance is measured by the Euclidean distance between the position of the current and previous time step of the vehicle's trajectory. Jumps up to $20.1 \mathrm{~m}$ are present when the vehicle navigation solution is corrected with a GPS fix.

Three coordinate systems are referenced for assessing the acoustic array data and for estimating the vehicle state. The coordinates are depicted in Figure 4-1. The origin of the first cartesian coordinate system is dock-centered. Positive y-direction is associated with North and positive $\mathrm{x}$-direction is associated with East. This system is used in programming the autonomous behaviors of the vehicle in MOOS-IvP. Consequently, the reported vehicle navigation data is in this system. The state estimation technique described in Section 4.2.5) 


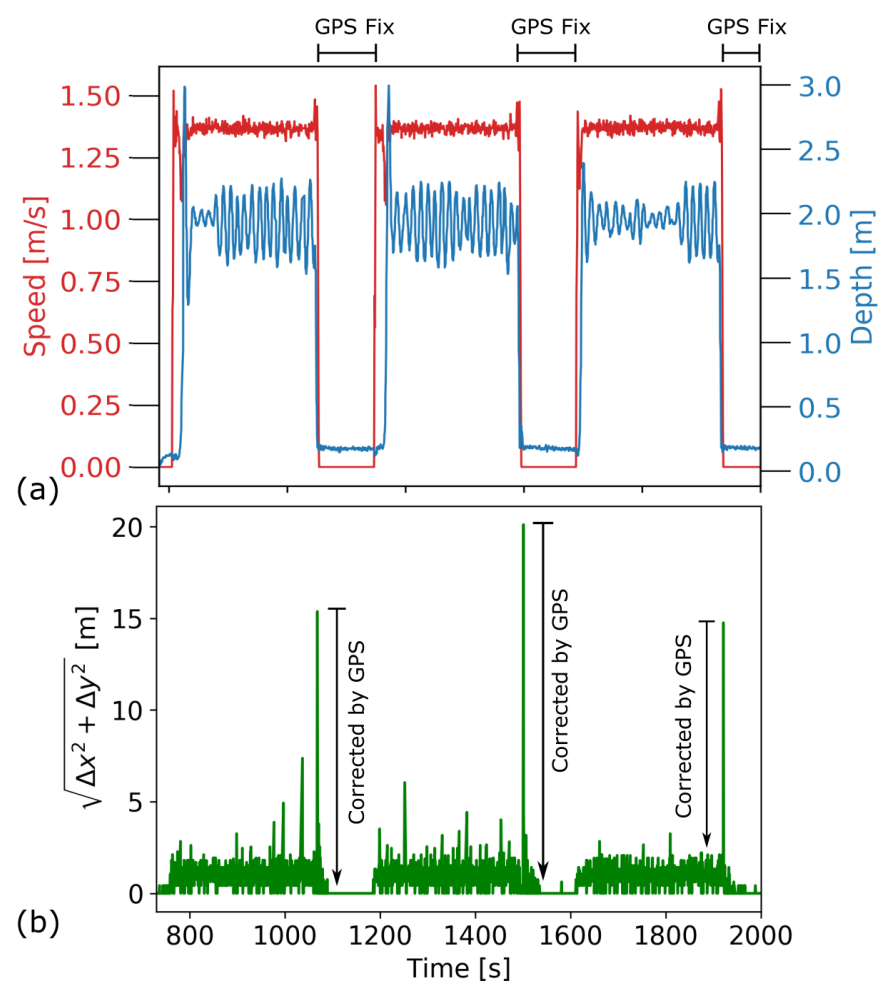

Figure 4-2: (a) Speed and depth of the vehicle over time during the Charles River field experiment. (b) Distance travelled by the vehicle between each time step shows significant jumps when a GPS fix is acquired.

also relies on this system for the kinematic model. The acoustic array polar coordinate system, also shown in Figure 4-1, is based off the location of the array. Range is the distance from the center of the line array to the vehicle position. Bearing is with respect to the line array and is positive counter-clockwise. The final coordinate system that is referenced is fixed to the UUV. For this analysis, the vehicle heading, which is normally positive clockwise from North, is redefined as positive counter-clockwise from East, as shown in the plot.

The acoustic array included 6 elements (HTI-96-MIN, $-165 \mathrm{~dB}$ re. $1 \mathrm{~V} / \mu$ Pa hydrophones) with a spacing of $0.075 \mathrm{~m}$. The data was sampled at $37500 \mathrm{~Hz}$. A spectrogram, created from the data of a single hydrophone element, of the entire experiment is in Figure 4-3. Before, during, and after the vehicle mission, boats passed by the acoustic array which resulted in broadband, interfering noise. By measuring ambient noise of marine traffic with and without the vehicle present, false detections and misses can be assessed. The vehicle performs its mission from 760 seconds to 1920 seconds. The strong tone at $17504 \mathrm{~Hz}$ originates from the BLDC motor inside the vehicle's propulsion system, and corresponds to the motor PWM 
switching frequency. Broadband acoustic noise from the vehicle is also observed, which can likely be attributed to a combination of machinery and cavitation noise.

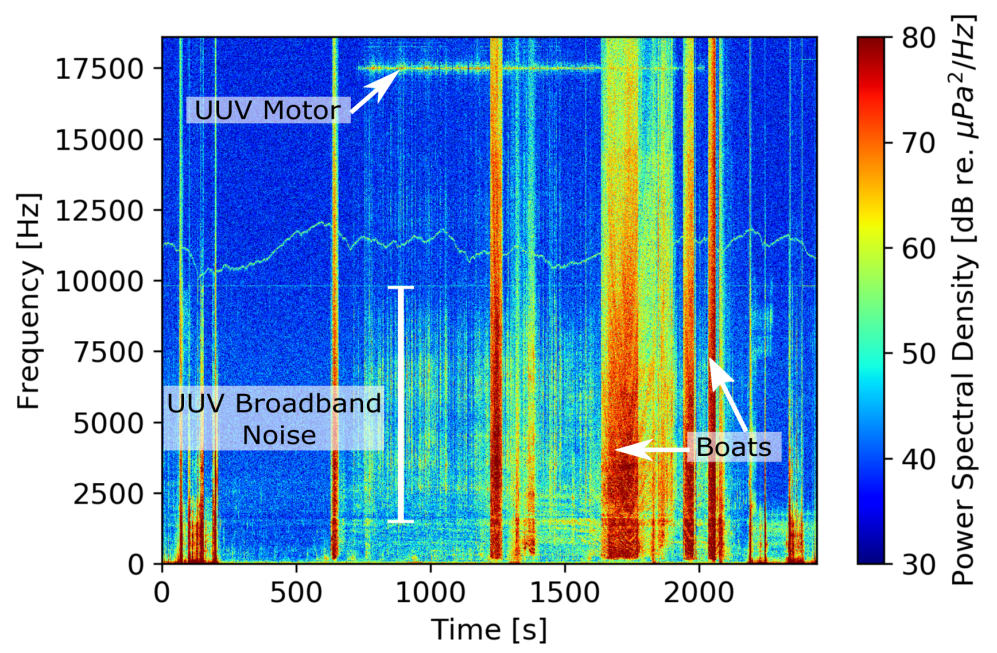

Figure 4-3: A spectrogram of the acoustic data collected in the Charles River field test experiment with radiating noise from the Sandshark UUV is presented. Strong tones from the motor inside the vehicle's propulsion system and broadband acoustic noise emitting from the vehicle are observable. During the experiment, boats passed by the robot, creating loud broadband interfering noise.

\subsubsection{Acoustic Array Data Analysis}

The signal processing chain for detecting, localizing, and tracking the vehicle from its acoustic signature with the stationary array is outlined in Figure 4-4. An overview of the process is as follows: (i) a bandpass filter is applied to isolate the vehicle signature; (ii) energy thresholding is implemented to declare the presence of a vehicle; (iii) conventional beamforming is performed to find the direction of arrival (DOA) of the vehicle's acoustic signature, as well as bearing rate; (iv) with a DOA estimate, the array is steered in the direction of the vehicle, which suppresses unwanted interference from passing boats; (v) with knowledge of the tonal features related to the motor design, the Doppler effect is measured to find range rate; (vi) the sideband spacing of the motor signature is measured to estimate the RPM of the vehicle propeller; (vii) finally, the measurements of bearing, bearing rate, range rate, and RPM are smoothed, then combined into an unscented Kalman filter (UKF) to predict the state of the vehicle over time. 


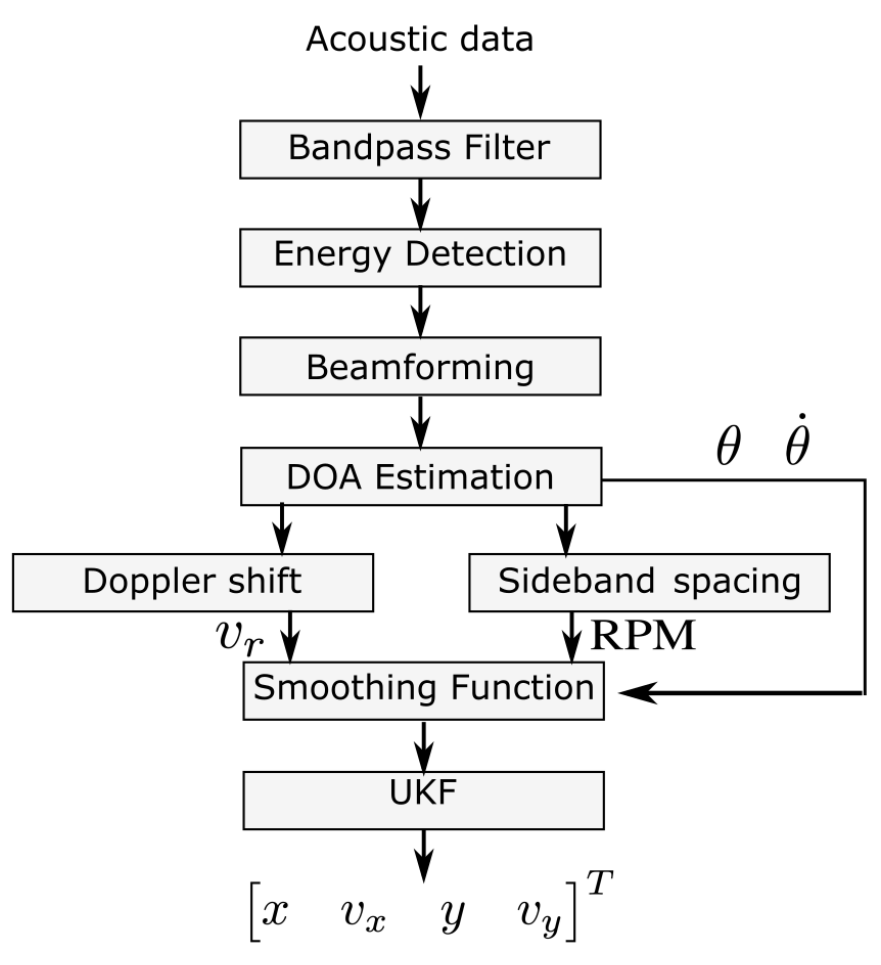

Figure 4-4: Signal processing chain for detection, localization, and tracking of the vehicle from motor noise.

The signal processing chain begins with detecting the presence of the vehicle. The signalto-noise ratio (SNR) of the target's acoustic signature determines whether the vehicle can be detected in the presence of passing marine traffic and at what ranges. The SNR of the vehicle at the receiving array is approximated by the sonar equation:

$$
S E=S L-T L-N L+A G-D T
$$

where $\mathrm{SE}$ is signal excess, the $\mathrm{SL}$ is the source level, TL is transmission loss, AG is array gain, NL is noise level at the receiver, and DT is detection threshold [145]. The SNR of the target is defined as $S L-T L-N L+A G$. If the SNR exceeds a specified DT, the UUV is declared present [1]. Likewise, when the SNR is below the DT, the target is declared absent. The ideal DT is selected such that the target is detected with low false alarms and high true detections. For the Charles River field test, the detection threshold is optimized through receiver operating characteristic (ROC) curves [146]. With knowledge of the vehicle's presence, ROC curves are produced by varying the detection threshold and calculating the number of false alarms and true detections. In order to evaluate false alarms, 
ambient noise data was collected from before and after the vehicle's autonomous missions. Therefore, we also have data where the UUV is absent, but boats are present. The sounds of boats passing could lead to a false UUV detection. For this analysis, the DT and SNR of the robot acoustic signature at the receiving array are evaluated in terms of signal energy over an observation period for a single hydrophone. First, a short-time discrete Fourier transform of the acoustic data from a hydrophone of 1 second segments with $50 \%$ overlap is calculated. Two detectors are compared to isolate acoustic noise radiating from the UUV: a detector with a bandpass filter at the motor signature $(17450-17550 \mathrm{~Hz})$ and a detector with a bandpass filter at the broadband vehicle noise $(1000-8000 \mathrm{~Hz})$. The filtered signal for each time segment is squared, integrated, and converted to decibels to measure energy for the detector. To account for fluctuations in the background noise, min-max normalization is applied to the energy result. After generating ROC curves for the two filters, the area under the curve (AUC) is used as a figure of merit to compare the performance of each detector [146].

Following detection, measuring the radiating acoustic noise of a vehicle from a line array provides three types of independent measurements which can be used for target motion analysis (TMA): bearing $\theta$, range rate $\dot{r}$, and vehicle speed $|\vec{v}|$. In this work, bearing rate $\dot{\theta}$ is also used as a measurement, derived from bearing with the finite difference method: $\frac{\theta_{t}-\theta_{t-1}}{\Delta t}$. First, we assume that we have determined that an unmanned underwater vehicle has been detected and that the PWM switching frequency and pole number inside the vehicle's propulsion system are known.

Beamforming on the radiating acoustic noise of the robot can be used to find the direction of arrival or bearing of the vehicle over time. To begin, we define the array manifold vector $\vec{v}_{\text {array manifold }}$ of uniform spacing steered to $\theta$, with the wavenumber $k_{s}=-\frac{2 \pi}{\lambda} \cos (\theta)$ :

$$
\vec{v}_{\text {array manifold }}\left(k_{s}\right)=\left[\begin{array}{lll}
e^{j(N-1) / 2) k_{s} d} & e^{j(N-1) / 2-1) k_{s} d} & \ldots
\end{array}\right]^{T}
$$

where $N$ is the total number of hydrophone elements, and $d$ is the element spacing [147]. The speed of sound used in this analysis is $c=1481 \mathrm{~m} / \mathrm{s}$. The array measurement data for hydrophone element $i, x_{i}[n]$, is divided into 1 second segments of data with $50 \%$ overlap. We take the discrete Fourier transform of the segmented data, $X_{i}[\omega]$, to apply a narrowband beamformer on each frequency bin of interest. The output beamformer for the frequency 
of interest $\omega$, steered to $k_{s}$ with uniform weighting is:

$$
Z\left[\omega, k_{s}\right]=\frac{1}{N} \vec{v}_{\text {array manifold }}\left(\omega, k_{s}\right)^{H} \vec{X}[\omega]
$$

The beamformer output for each frequency bin of interest is averaged, $Z\left[k_{s}\right]$. The look angle that produces the maximum beamformer output power is the estimated direction of arrival [148]:

$$
\hat{\theta}=\underset{\theta}{\operatorname{argmax}}\left|Z\left[k_{s}(\theta)\right]\right|^{2} .
$$

Angles from $0^{\circ}$ to $180^{\circ}$ at $1^{\circ}$ intervals were considered. Two frequency bands were evaluated for finding the direction of arrival of the robot: 1000-8000 Hz at $250 \mathrm{~Hz}$ intervals for the broadband radiating noise from the vehicle and $17450-17550 \mathrm{~Hz}$ at $1 \mathrm{~Hz}$ intervals for capturing the motor signature. The angle resolution of the beamformer output improves with an increase in frequency. For context, the half-power beamwidths for this array setup steered to broadside are $9.6^{\circ}$ for $17500 \mathrm{~Hz}$ (motor signature range) and $21.1^{\circ}$ for $8000 \mathrm{~Hz}$ (upper bound of broadband UUV noise) [147]. Beamforming on the motor signature produces a narrower beamwidth, however, the spatial aliasing cutoff for this array spacing is $9873.3 \mathrm{~Hz}$. Therefore a grating lobe is present in the motor signature beamforming results. As shown in Figure 4-1, the coordinate system of the bearing and bearing rate measurement is with respect to the array position and orientation, where bearing is positive clockwise, and range is the euclidean distance to the vehicle from the array position. Note, that for TMA, these measurements are converted to positive counter-clockwise $\theta=-\theta_{\text {measured }}$.

With the estimated bearing over time, the array is steered in the direction of the robot to improve the SNR of the vehicle signature. Using the array output, range rate and speed of the vehicle are measured using prior knowledge of the motor acoustic signature. The Doppler shift of the PWM switching frequency of the motor is used to determine the range rate of the vehicle. The significance of a constant, high frequency tone radiating from the vehicle is that we can observe a Doppler shift, even when the vehicle is slow-moving.

The expression for the observed tone emitting from the vehicle (moving source) $f_{\mathrm{PWM}}$, observed at the stationary array due to Doppler shift is:

$$
f_{\mathrm{PWM}, \text { observed }}=\frac{c}{c \pm v_{\mathrm{UUV}}} f_{\mathrm{PWM}}
$$


where $c$ is the speed of sound and $v_{\mathrm{UUV}}$ is the relative speed of the vehicle. If the vehicle moves away from the array, the denominator expression is $c+v_{\mathrm{UUV}}$, and the observed frequency decreases. If the vehicle approaches the array, the denominator expression is $c-v_{\mathrm{UUV}}$, and the observed frequency increases [149].

We also measure the sideband spacings around the thruster PWM switching frequency to find RPM. The relationship between RPM and the sideband spacings is:

$$
f_{\mathrm{m}}=f_{\mathrm{s}} p
$$

where the $f_{m}$ is the sideband spacing, $p$ is the number of permanent magnetic poles, and $f_{s}$ is the shaft rotation frequency [48]. The mapping of RPM to speed is derived from a vehicle model, described in Section 4.2.4.

The acoustic array data analysis results in four measurements - bearing $\theta$, bearing rate $\dot{\theta}$, range rate $v_{r}$ and speed $|\vec{v}|$ which are used for predicting the vehicle position over time. The measurement vector at discrete time $\mathrm{k}$ is:

$$
\vec{z}_{k}=\left[\begin{array}{c}
v_{r} \\
|\vec{v}| \\
\theta \\
\dot{\theta}
\end{array}\right]_{k}
$$

The measurements are interpolated to be on the same time scale at 1.2 second time intervals. A third-order Savitzky-Golay smoothing filter of window with size 45 of samples is applied to smooth any jumps or discontinuities.

In this dataset, temporal and spatial aliasing occurred. However, the effect of aliasing was accounted for in the Doppler measurements and beamforming results. The Doppler shifted frequency of $f_{\mathrm{PWM}}$ is above the Nyquist frequency. Thus, when estimating the range rate of the vehicle, one must account for the affect of the aliasing: $f_{\mathrm{PWM}, \text { aliased,Doppler }}=f_{s r}-\left(f_{\mathrm{PWM}}+\Delta f\right)$ where $f_{s r}$ is the sampling rate and $\Delta f$ is the Doppler shift. When the range rate increases (e.g. when the vehicle is moving away from the array), the true Doppler shift results in a decrease in frequency, but the aliased signal shows an increase in frequency.

In accounting for temporal aliasing, beamforming analysis was performed on the aliased, perceived frequency since it is known that the frequency of the reconstructed signal is 
$f_{\mathrm{PWM}}$, aliased $=\left(f_{s r}-f_{\mathrm{PWM}}\right)$. Beamforming on a time signal that is aliased will also result in a phase reversal: a beam is present at a symmetric angle, $\theta_{r}$ to the intended steering direction, $\theta_{\text {steered }}$, such that $\theta_{\text {steered }}=180-\theta_{r}$. Spatial aliasing occurred in addition to temporal aliasing because the array element spacing was greater than $\lambda / 2$. A grating lobe is present in the beamforming results. However, with knowledge of the true position of the robot from the vehicle's navigation data, we are able to determine the direction of arrival from the grating lobe.

\subsubsection{Estimating the UUV Speed Prediction Model}

In order to estimate the speed of the vehicle from RPM measurements, we assume a linear model of the robot:

$$
u=\alpha \mathrm{RPM}
$$

where $u$ is the forward speed of the robot, or $|\vec{v}|$. In practice, the $\alpha$ parameter is determined by a recursive least squares system identification algorithm, using the vehicle's propeller rotation rate and IMU-based navigation data of forward speed, obtained from a past mission. The forward speed is derived from the 3-axis gyroscope, 3-axis accelerometer, and 3-axis magnetometer inside the IMU, as well as speed-over-ground estimated from propeller RPM and corrected by GPS measurements [38]. To identify the system model, the IMU-based forward speed estimation, $u$, is assumed as the ground-truth, and is compared against the model predicted speed $\hat{u}$ in the cost function:

$$
V(\alpha)=\frac{1}{N} \sum_{t=1}^{N}|u-\hat{u}|^{2}
$$

The $\alpha$ parameter is estimated by minimizing the cost function given in Equation 4.9 using recursive least squares:

$$
\alpha^{*}=\underset{\alpha}{\arg \min }\left[\frac{1}{2 N} \sum_{t=1}^{N}\left|u_{(t)}-\alpha_{(t-1)} \mathrm{RPM}_{(t)}\right|^{2}\right]
$$

The optimal model parameter, $\alpha^{*}$ is used in the UKF results in the following sections. We refer to $\alpha^{*}$ as the Dynamic Model Prediction. For implementing this method on other UUVs and in other environments, it is also important to consider model mismatch of the derived dynamic parameter $\alpha$. The vehicle model could be unknown, or external forces 
such as currents could influence the model error. The sensitivity of the UKF result to this parameter is evaluated in Section 4.3.5. For various values of $\alpha$ above and below $\alpha^{*}$, (within $10 \%$ of $\alpha^{*}$ ), the state variable error of the UKF result is recorded and compared.

In this work, the true propeller RPM from the vehicle on-board navigation system was unavailable at the time of the experiment. Therefore, for the purpose of demonstrating how this technique can be incorporated into target motion analysis, the IMU-based forward speed of the vehicle and acoustic measurements of propeller RPM are used in the system identification.

\subsubsection{Unscented Kalman Filter Implementation}

For the passive UUV tracking application, the observation equations, which transform the state variables of the vehicle into the expected acoustic measurements of bearing, bearing rate, range rate, and RPM, are nonlinear. In this work, the unscented Kalman filter (UKF) was selected over the extended Kalman filter and Particle Filter, alternative nonlinear state estimation methods. The UKF possesses better stability over the extended Kalman filter $[77,78]$ and runs more efficiently than the Particle Filter $[79,80]$.

The vehicle state variables are selected to completely describe the vehicle's state over time at a constant depth:

$$
\vec{x}_{\mathrm{UUV}, k}=\left[\begin{array}{c}
x \\
v_{x} \\
y \\
v_{y}
\end{array}\right]_{k} \text {. }
$$

A constant-velocity, linear process model was used for describing the vehicle's motion for the prediction step of the UKF:

$$
\vec{x}_{\mathrm{UUV}, k+1}=\left[\begin{array}{cccc}
1 & \Delta t & 0 & 0 \\
0 & 1 & 0 & 0 \\
0 & 0 & 1 & \Delta t \\
0 & 0 & 0 & 1
\end{array}\right]\left[\begin{array}{c}
x \\
v_{x} \\
y \\
v_{y}
\end{array}\right]_{k} .
$$

The expected acoustic measurements from this prediction are calculated using nonlinear 
observation equations. These equations include converting the cartesian vehicle state to polar coordinates. Range, $r$, between the vehicle and array is calculated with knowledge of the array position:

$$
r=\sqrt{\left(x-x_{\text {array }}\right)^{2}+\left(y-y_{\text {array }}\right)^{2}}
$$

Heading is also calculated via $\phi=\tan ^{-1}\left(v_{y} / v_{x}\right)$. With range, the expected range rate is:

$$
v_{r}=\frac{1}{r}\left[\left(x-x_{\text {array }}\right) v_{x}+\left(y-y_{\text {array }}\right) v_{y}\right] .
$$

Bearing of the vehicle relative to the array accounts for the orientation of the array, an offset $\theta_{0}$ :

$$
\theta=\tan ^{-1}\left(\frac{y-y_{\text {array }}}{x-x_{\text {array }}}\right)-\theta_{0}
$$

Speed is determined from the velocity components: $|\vec{v}|=\sqrt{v_{x}^{2}+v_{y}^{2}}$. Two forms of bearing rate are used to avoid discontinuities when $\sin (\theta)$ or $\cos (\theta)$ approach zero:

$$
\begin{aligned}
& \dot{\theta}=\frac{v_{\mathrm{x}, \text { array }}-v_{r} \cos (\theta)}{-r \sin (\theta)}, \\
& \dot{\theta}=\frac{v_{\mathrm{y}, \text { array }}-v_{r} \sin (\theta)}{r \cos (\theta)} .
\end{aligned}
$$

The projected $v_{x}$ and $v_{y}$ into the array coordinate system are:

$$
\begin{aligned}
& v_{\mathrm{x}, \text { array }}=|\vec{v}| \cos \left(\phi-\theta_{0}\right) \\
& v_{\mathrm{y}, \text { array }}=|\vec{v}| \sin \left(\phi-\theta_{0}\right) .
\end{aligned}
$$

\subsection{Results}

\subsubsection{Passive Acoustic Detection}

The first objective in the Charles River field test analysis is to determine if the UUV can be detected from its radiating noise in the presence of boat traffic. Two energy detectors are evaluated for this purpose. A detector with a bandpass filter at $1000-8000 \mathrm{~Hz}$ is applied to isolate broadband acoustic noise emitted from the vehicle. The second detector evaluated has a bandpass filter at $17450-17550 \mathrm{~Hz}$ to isolate the motor acoustic signature. The ideal threshold for energy detection is identified through ROC curve analysis, plotted in Figure 4- 
5.

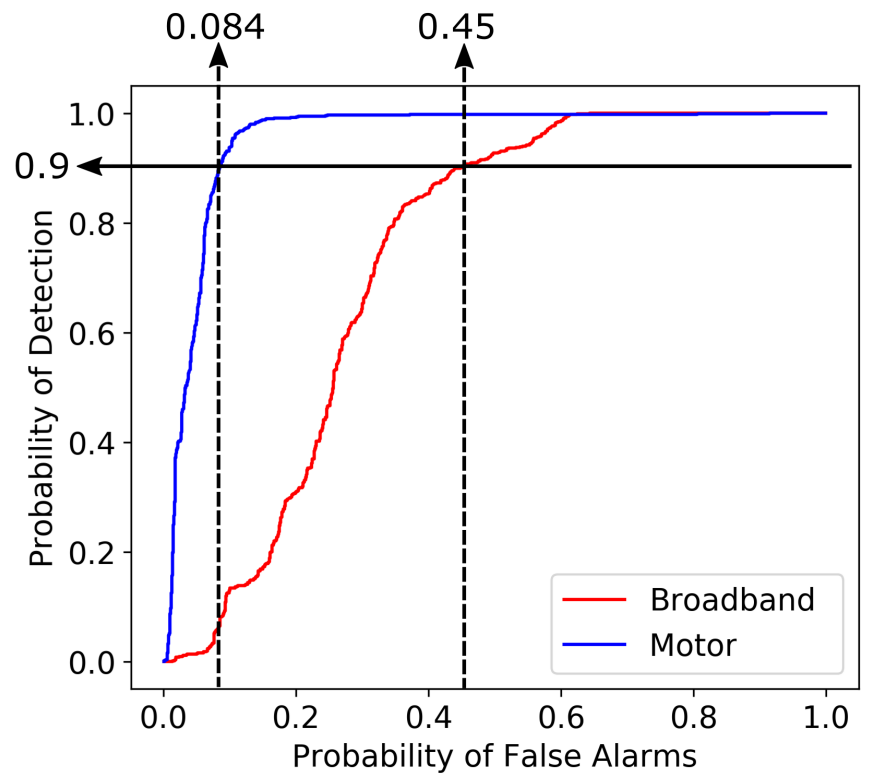

Figure 4-5: ROC curve analysis is performed to compare detecting the UUV in the Charles River field test with two energy detectors. The first detector uses a bandpass filter on broadband noise and the second detector uses a bandpass filter on the motor signature noise.

Figure 4-5 highlights the significant improvement of filtering on the motor signature. For a detection rate of 0.9 , the motor signature yields 0.084 false alarms, while broadband produces 0.45 false alarms. Furthermore, the area under the curve for the motor signature is 0.954 and for the broadband noise is 0.732 . With a set threshold for $90 \%$ detection probability, the performance of the two detectors are compared over the time in Figure 4-6. The number of false alarms due to passing boats are significantly reduced with the motor noise filter.

\subsubsection{Direction of Arrival Estimation}

In order to estimate the bearing and bearing rate of the vehicle, the direction of arrival (DOA) of the UUV acoustic signature is first estimated by applying conventional beamforming on two different frequency bands, the motor signature and broadband vehicle noise. The bearing time recordings (BTR) for the two different cases are plotted in Figures 4-7b and 4-7d. In each of the BTR plots, the power is normalized for each time segment, and a white dashed line is drawn to show the expected bearing of the vehicle over time, taken 


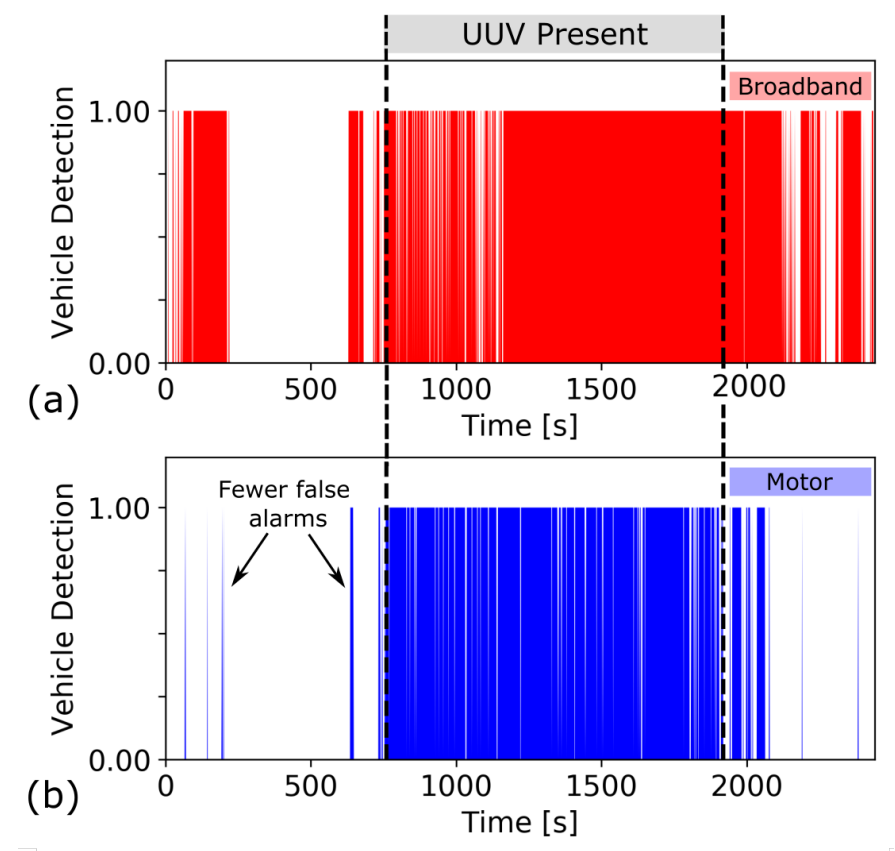

Figure 4-6: Passive acoustic detections of the UUV are plotted over time with 1 corresponding to a detection and 0 corresponding to no detection. The UUV is present from 760-1920 seconds. Two energy detectors are compared with different bandpass filters for isolating broadband vehicle noise and the motor acoustic signature at (a) $1000-8000 \mathrm{~Hz}$ and (b) $17450-17550 \mathrm{~Hz}$ respectively. The threshold for both detectors is set at $90 \%$ probability of detection.

from the IMU-based navigation solution of the robot. Boats passed by the vehicle and created loud, interfering broadband noise during the following four periods: 1219-1268 seconds, 1289-1373 seconds, 1625-1891 seconds, and 1926-1950 seconds. During these periods, additional bearing tracks are present in the BTR plots.

The corresponding DOA plots for broadband and motor noise are plotted in Figures 4-7c and 4-7e. The DOA is derived from measuring the maximum beamformer output power over time. In these plots, the measured bearing is compared to the true bearing, which is obtained from the vehicle's on-board navigation solution for position, and the array position. Due to the spacing of the array, spatial aliasing occurred, which created a grating lobe in Figure 4-7d. However, with the expected bearing derived from the vehicle's navigation system, the true track could be identified.

As shown in Figure 4-7, beamforming on the high frequency of the motor signature results in a narrower beamwidth compared to the beamforming on the broadband noise of the vehicle. Furthermore, the DOA estimate from beamforming on the motor signature 
is superior to broadband vehicle noise since the measurement has higher SNR. For the extent of the experiment where the UUV was present, the resulting DOA estimate error for broadband noise was $28.9^{\circ}$ and the motor signature was $6.9^{\circ}$, which is a $76 \%$ decrease in error. With the DOA estimate from beamforming on the motor signature, this result is smoothed and used to steer the array in the direction of the vehicle over time.
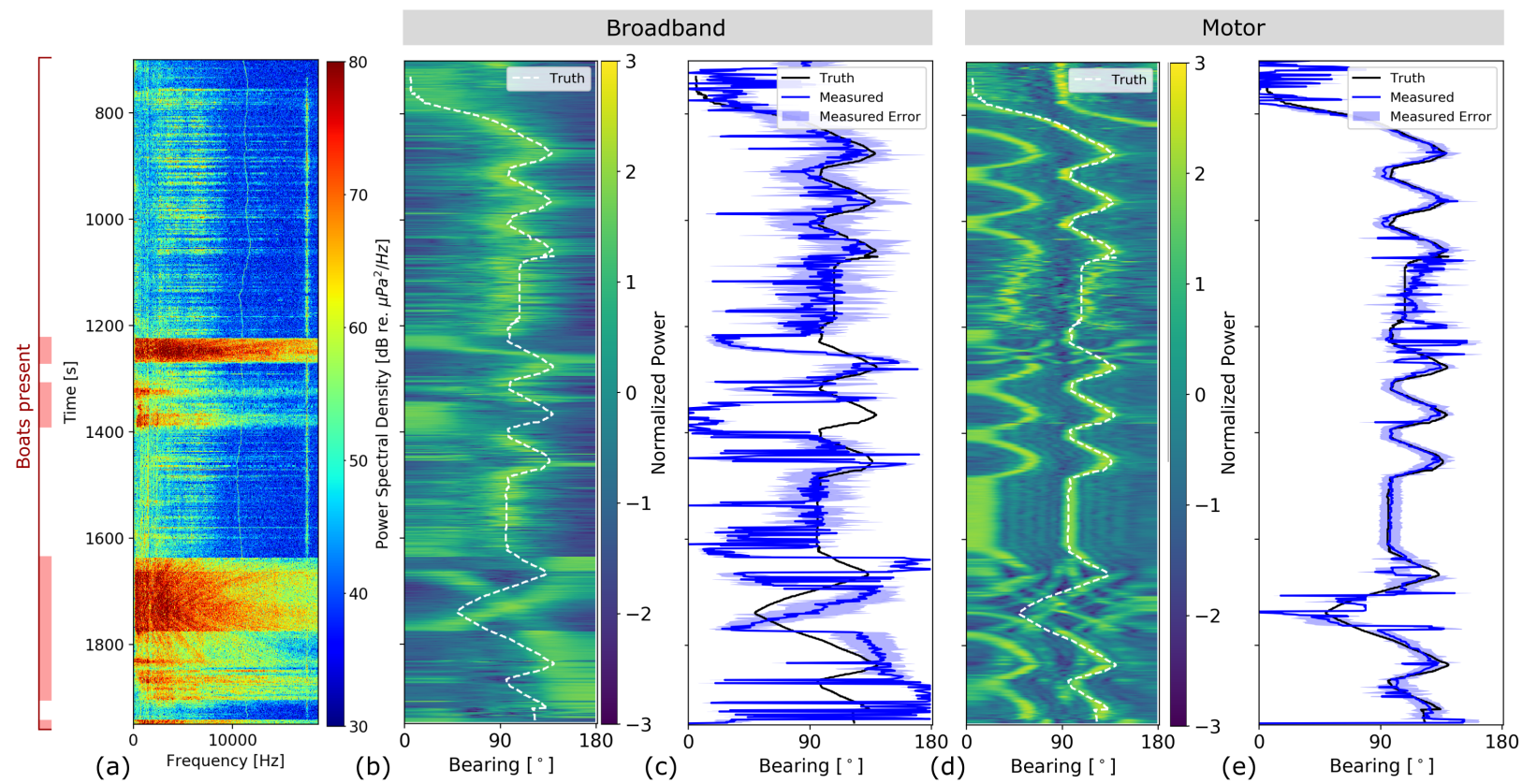

(a) Frequency $[\mathrm{Hz}]$

(b) Bearing $\left[{ }^{\circ}\right]$

(c) Bearing $\left[{ }^{\circ}\right]$

(d) Bearing $\left[{ }^{\circ}\right]$

(e)

Figure 4-7: Considering only the period of the experiment where the UUV is present, the acoustic data collected on the radiated noise of the vehicle from the line array is analyzed. (a) The acoustic data from a single hydrophone is used to generate a spectrogram. A timeline of when boats are present is denoted in red to the left of the spectrogram. Bearing time recordings of the radiating acoustic noise of the UUV (b) broadband noise and (d) motor noise are compared. The respective direction of arrival plots are in (c) and (e).

\subsubsection{Range Rate and RPM}

The range rate and RPM measurements from the motor signature are obtained from the steered array output. The Doppler effect of the PWM switching frequency is observable from both the fin servomotors and thruster, as shown Figure 4-8 and Figure 4-9 respectively. The Doppler shift of the PWM switching frequency corresponds to the relative velocity of the vehicle with respect to the stationary array (the range rate).

The vehicle follows a loiter pattern with periodic pauses to acquire a GPS fix at the 
surface. As demonstrated in Figure 4-8, the vehicle moves closer and further from the array and the perceived tone at the PWM switching frequency changes pitch. When the vehicle is stationary for a GPS fix, the fin servomotors are engaged in a holding position and the perceived tone is constant. Also in Figure 4-8a, a black dashed line of the expected perceived frequency is plotted against the spectrogram, determined from the vehicle navigation data. The measured value of the perceived tone was the frequency with the maximum power for each time segment. Figure 4-8b is a comparison of the expected and measured tone at the switching frequency over time. Using Equation 4.5 for the Doppler shift, and assuming the true tone of the switching frequency is $17504 \mathrm{~Hz}$, range rate is calculated from the measured perceived tone. Considering the entire period of the experiment where the UUV is present, the average error in range rate is $0.329 \mathrm{~m} / \mathrm{s}$.

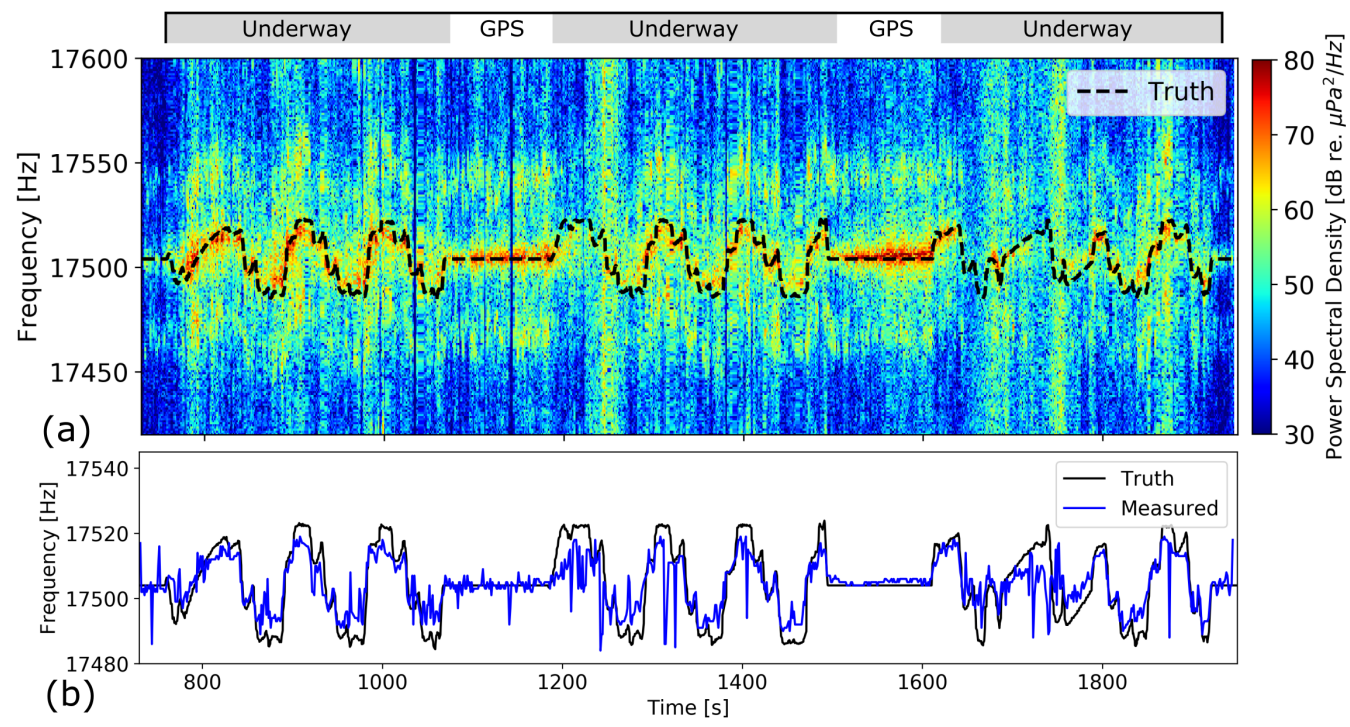

Figure 4-8: (a) The Doppler shift of the fin servomotor's PWM switching frequency is observed in the spectrogram. Above the spectrogram is a timeline of when the vehicle is underway, and when the vehicle has stopped for a GPS fix. The perceived tone is constant when the vehicle is stationary for a GPS fix, and changes frequency as the vehicle moves closer and further from the array. (b) The measured and expected perceived tone of the switching frequency from the stationary array are compared.

By using peak-finding and symmetry, the sideband spacings centered at the PWM switching frequency were measured. With knowledge of the vehicle's motor pole number, the RPM was estimated using equation 4.6. Figure 4-9a is a spectrogram centered at the tones radiating from the motor. During the segments 1219-1268 seconds and 1625-1891 
seconds, boats pass and the RPM measurement is difficult to acquire. In Figure 4-9b, the expected and measured RPM are well-aligned except when there is interference from boats. The true propeller rotation rate is calculated from the IMU-based navigation solution of the forward speed of the robot. Speed is converted to RPM using a linear conversion provided by the robot manufacturer. In comparison to the acoustic measurement of propeller rotation rate, the average error of the entire experiment is $216 \mathrm{rpm}$. When considering only the periods where no boats are present, the propeller rotation error decreases to $79 \mathrm{rpm}$.

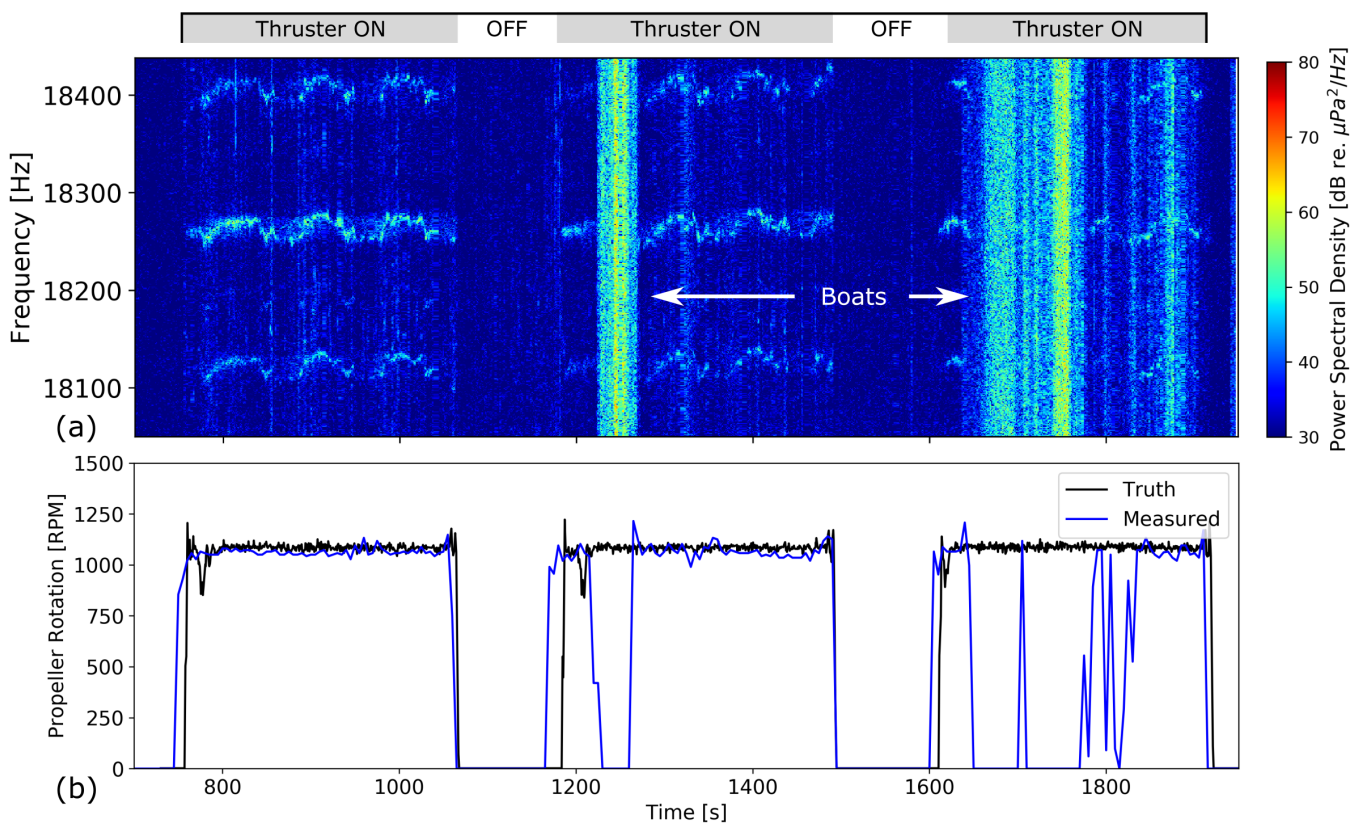

Figure 4-9: (a) Sidebands centered on the PWM switching frequency of the thruster motor are observed in the spectrogram. Above is a timeline of when the thruster is on (the vehicle is underway) and the thruster is off (the vehicle is at the surface for a GPS fix). Broadband noise interference from boats passing by the UUV is also annotated. (b) Propeller rotation of the UUV is measured over time from the sidebands and compared to the true RPM of the vehicle.

\subsubsection{Localization and Tracking}

A birds-eye view of the IMU-based navigation solution (used as the truth) of the vehicle position over time is plotted in Figure 4-10a for the Charles River test. The vehicle trajectory is plotted with respect to the array polar coordinate system. In Figure 4-10b, the UKF filtered result of the vehicle position is plotted over time. The UKF result fuses the passive acoustic, remote measurements of bearing, bearing rate, RPM, and range rate. In order to 
interpret the estimated vehicle track, the individual state variables predicted from the UKF are plotted against the true values in Figure 4-11. With high-fidelity bearing measurements, the UKF consistently and accurately predicts the bearing of the vehicle. The accuracy for estimating range deteriorates when boats pass by - particularly the segment of 1625-1891 seconds where we observe a jump in range in Figure 4-10b and Figure 4-11a. The poor range estimate appears to correspond to the RPM measurement error increase when boat noise interferes.

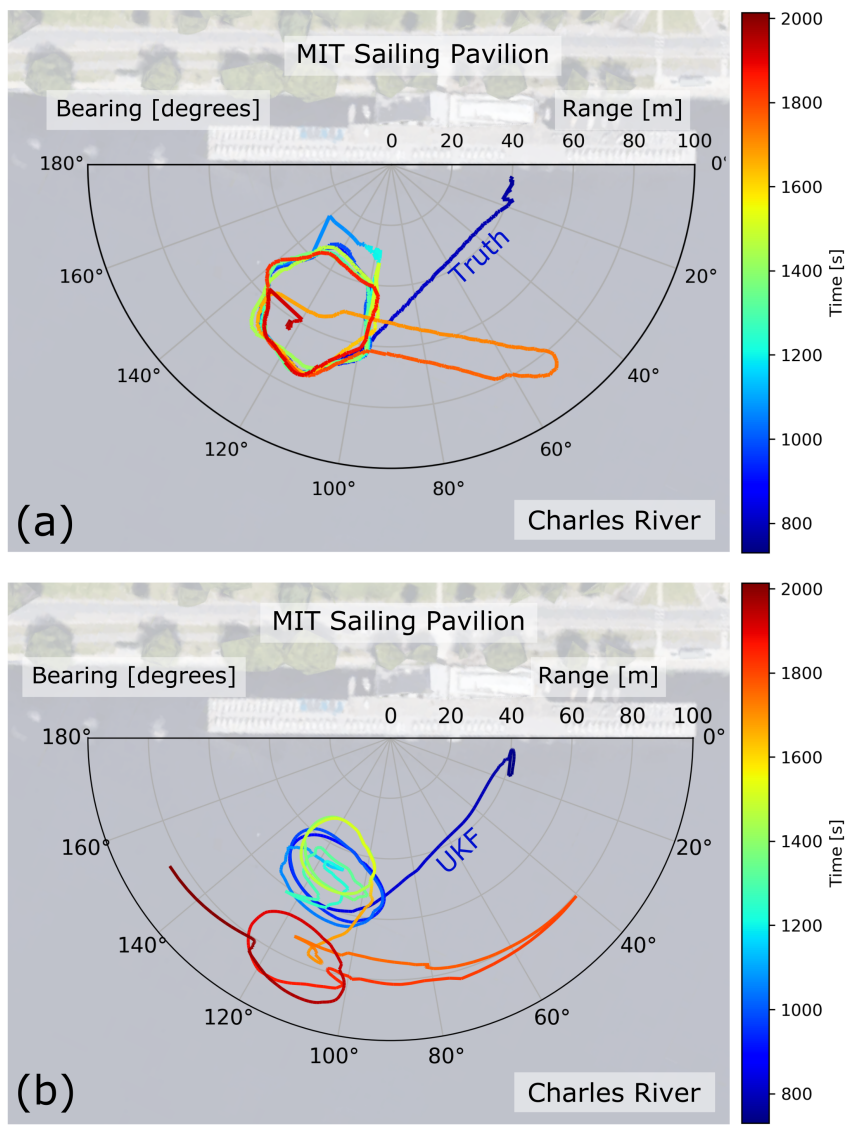

Figure 4-10: (a) The true vehicle position, taken from the IMU-based navigation solution of the robot from the Charles River field test, is plotted over time. The coordinate system is with respect to the stationary, dock-mounted array. For comparison, the predicted vehicle position from fusing passive acoustic measurements with a UKF is plotted in (b).

To illustrate the effect of interfering ambient noise on the tracking results, bar plots of the average error for each state variable from this experiment are shown in Figure 4-12. The colors of yellow and green in the bar plots refer to the error when boats are present and no 

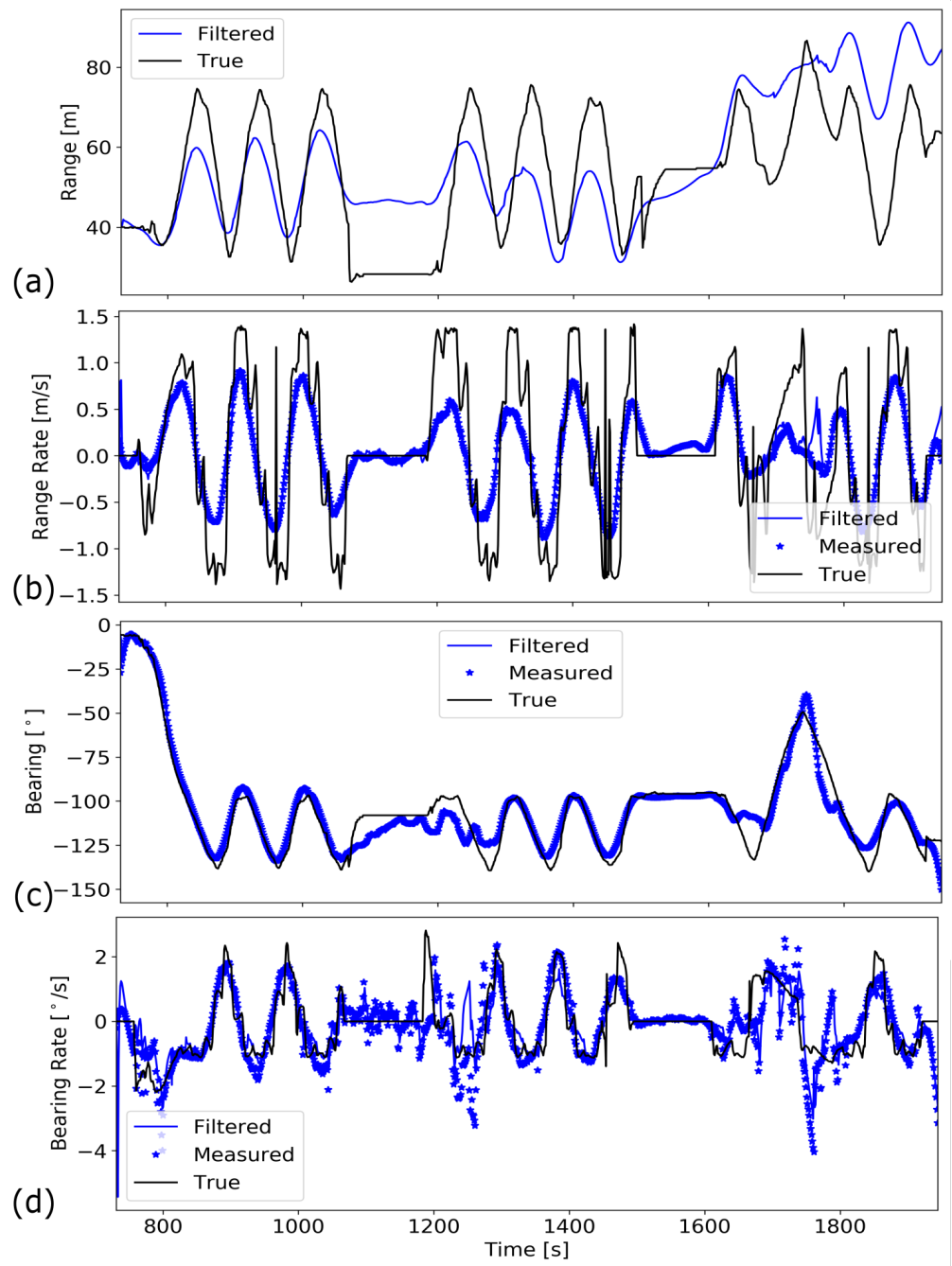

Figure 4-11: The UKF-predicted and true state variables of the UUV from the field test are plotted individually: (a) range, (b) range rate, (c) bearing, and (d) bearing rate.

boats are present respectively. The columns of Figure 4-12 refer to running the UKF with a different number of measurements: (i) bearing and range rate (traditional TMA), (ii) bearing, range rate, and speed, and (iii) bearing, bearing rate, range rate, and speed. The rows of Figure 4-12 are the four state variables, where the y-axis is the average absolute error for each variable. As expected, across all four state variables, the trend in adding measurements decreases error in the UKF state estimate. It is also apparent, across all cases, that the state estimation error increases in the presence of loud interfering noise of boats.

Notably, the addition of the RPM measurement to traditional TMA with bearing- 
Doppler measurements results in a significant decrease in error. The drop in error is especially pronounced in bearing rate (Figures $4-12 \mathrm{~b}$ and $4-12 \mathrm{f}$ ) and range rate (Figures 4-12d and $4-12 \mathrm{~h}$ ). The baseline case for tracking the UUV with bearing-Doppler yielded the following state variable errors: $34.6 \mathrm{~m}$ for range, $12.6^{\circ}$ for bearing, $2.34 \mathrm{~m} / \mathrm{s}$ for range rate, and $10.1^{\circ} \mathrm{s}$ for bearing rate. In contrast, using all four measurements of bearing, bearing rate, range rate, and RPM for the UKF yielded errors of: $11.1 \mathrm{~m}$ for range, $5.66^{\circ}$ for bearing, $0.38 \mathrm{~m} / \mathrm{s}$ for range rate, and $0.64 \% \mathrm{~s}$ for bearing rate. This corresponds to a reduction in error by $55 \%, 93 \%, 68 \%$, and $83 \%$ for bearing, bearing rate, range, and range rate respectively. We expect these results to further improve when the constant-velocity process model reflects the actual behavior of the UUV, such as when the robot moves in long, straight line segments.

\subsubsection{Robustness of RPM-Speed model}

The sensitivity of the UKF tracking solution to the dynamic parameter $\alpha$ (which linearly maps RPM to vehicle speed) is assessed in Figure 4-13. While bearing, bearing rate, propeller rotation rate, and range rate of the target can be directly measured acoustically, range to the vehicle cannot. Therefore, we evaluate sensitivity of the range estimate to an unknown dynamic vehicle model. Within $30 \%$ of the dynamic model prediction $\left(0.00129 \frac{\mathrm{m} / \mathrm{s}}{\mathrm{RPM}}\right)$, the range error increased by approximately $4 \mathrm{~m}$ : from $11.1 \mathrm{~m}$ to $14.8 \mathrm{~m}\left(0.000903 \frac{\mathrm{m} / \mathrm{s}}{\mathrm{RPM}}\right)$ and $14.9 \mathrm{~m}\left(0.001677 \frac{\mathrm{m} / \mathrm{s}}{\mathrm{RPM}}\right)$. The mean absolute error of the UKF state variable result is calculated and plotted for a range of $\alpha$ values in Figure 4-13. The dynamic model prediction $\alpha^{*}$ minimizes the mean absolute error of the state variable.

\subsection{Conclusion}

In this work, an unmanned underwater vehicle (UUV) was passively detected and tracked by high-frequency acoustic noise radiating from the fin actuator motors and thruster motor, despite loud interference from boat traffic. Sound originating from the motors is not only high amplitude, but also has the following advantages for passive acoustic detection, classification, localization, and tracking: high frequency (leading to an observable Doppler shift and narrower beamwidth for direction of arrival), and the additional measurement of RPM. In comparison to traditional bearing-Doppler target motion analysis (TMA), includ- 
ing the measurement of propeller rotation rate dramatically improves the tracking solution. We also demonstrated that an unknown dynamic vehicle model within $30 \%$ of the true speed to RPM mapping results in a minimal change to the unscented Kalman filter range estimate. This is important for applying this method to vehicles where the dynamic model is unknown, or when external forces are present such as water current.

Immediate next steps in improving UUV detection from motor noise include leveraging knowledge of the power spectral density of the motor signature, such as with the optimum frequency shaping Eckart filter [145]. Beamforming on the motor signature could be improved by incorporating adaptive beamforming [147]. In order to account for ocean currents when tracking the UUV, the velocity of the water current can be estimated as a state variable in the unscented Kalman filter or other state estimation technique [125-127]. In our implementation, we assumed the vehicle was moving at a constant-velocity, but in the experiment, the robot was moving in a circle. Therefore, we expect the tracking results to improve for future experiments involving a robot that moves in straight-line segments. To confirm this, we recommend additional experiments with other common vehicle behaviors, such as a lawn-mower pattern.

Future work on this topic should include evaluating this method on other UUVs. We hypothesize that robots which rely on mass-manufactured permanent magnet AC synchronous motors, also produce tones at the PWM switching frequency which can be detected and tracked. The impact of motor noise mitigation techniques on the overall noise of the vehicle, such as skewing the permanent magnets inside the motor, should be investigated. Decreasing the acoustic noise originating from the motor will in turn affect the accuracy of tracking the vehicle.

An important consideration for implementing this method into passive sonar systems is the limitation of range, since high frequency tones are subject to attenuation. Therefore, future research should include acoustic propagation modelling of UUV motor noise. Given the known acoustic signature of the motor, the range limitations of this method can be simulated and assessed for different environment conditions. In order to accurately evaluate the range limitations, though, additional measurements of the UUV should be taken to find the true source level and radiation pattern of the motor noise.

In conclusion, we evaluated and demonstrated UUV passive acoustic detection, localization, and tracking from the high-frequency, high amplitude tones originating from the 
fin and thruster motors inside the vehicle. This method can be incorporated into passive sonar systems for port and harbor security, as well as for situational awareness from ships and submarines. 


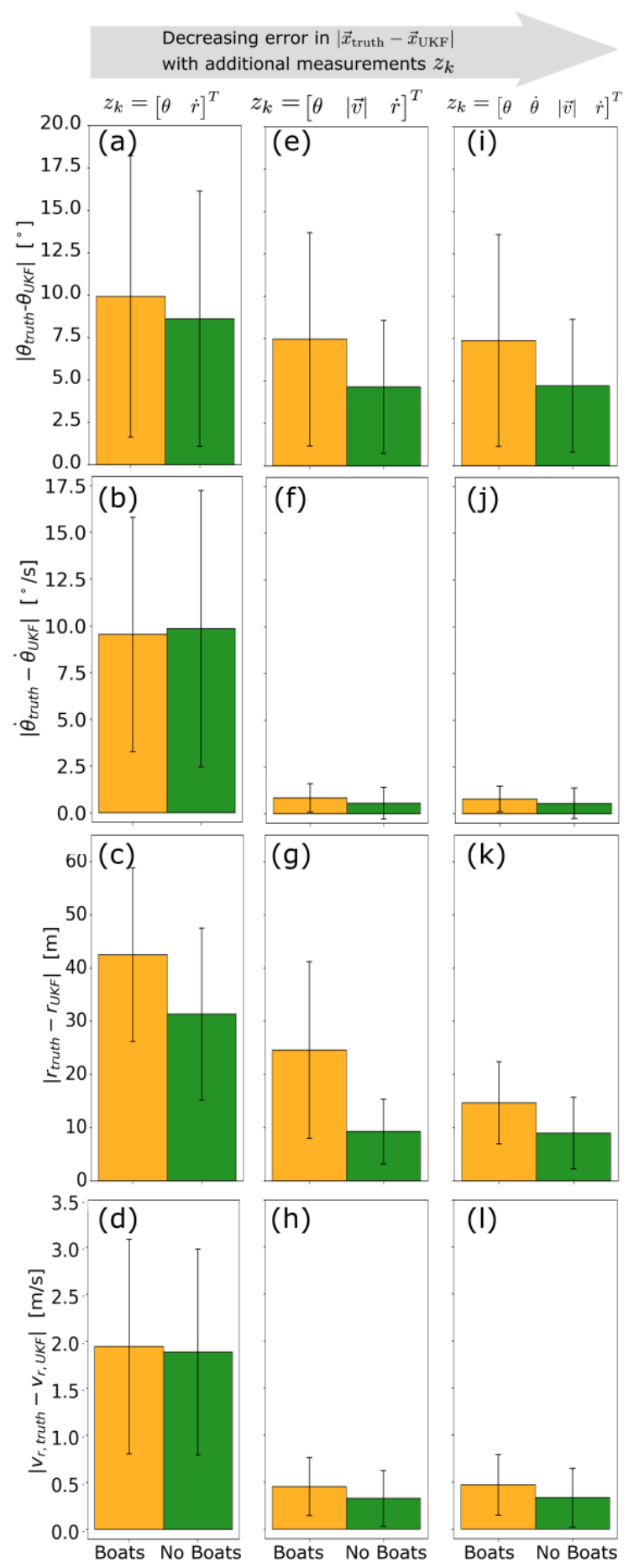

Figure 4-12: Error, defined as the mean absolute difference, for the predicted UKF state variables are presented. The columns represent the acoustic measurements used in the UKF. The first column is the baseline case for passive UUV tracking with bearing-Doppler measurements. As the number of available measurements increases, the error in the UKF-predicted state variables decreases. 


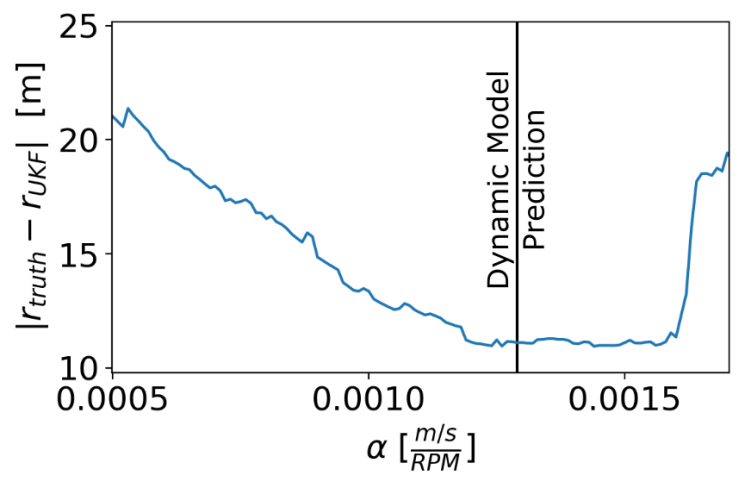

Figure 4-13: The resulting average error of the UKF prediction for range is plotted for different values of the RPM-speed mapping $(\alpha)$. The dynamic model prediction for the Sandshark UUV $\alpha^{*}$, which is used in the UKF analysis, aligns with the minimum state variable error. 


\section{Chapter 5}

\section{Conclusion}

This thesis work contributes to the field of unmanned underwater vehicle (UUV) research by characterizing high-frequency acoustic noise in UUVs. I discovered that the unique acoustic signature of off-the-shelf BLDC motors - which a significant number of UUVs rely onprovides high SNR measurements of bearing, propeller rotation rate, and range rate which can be used for passive acoustic tracking. I validated that it is possible to passively track an unmanned underwater vehicle from its high-frequency motor noise in the presence boat traffic in shallow-water experiments. In addition, I demonstrated that bearing-DopplerRPM measurements outperform traditional bearing-Doppler target motion analysis in the unscented Kalman filter implementation. These findings are significant for improving UUV localization and tracking, and can be incorporated into passive sonar systems.

An important consideration before integrating this method into passive acoustic surveillance systems is taking the environmental effects on the acoustic measurements into account. In this final chapter I discuss the range limitations of this method, as well as other potential challenges when working in the ocean. Lastly, I end with future improvements and directions to take this work.

\subsection{Implementation in the Ocean Environment}

The acoustic measurements collected in the Charles River field tests exhibited some common issues of working in a complex underwater environment such as loud ambient noise, but in some aspects, had significant advantages. The river, with a shallow depth of approximately 
$6 \mathrm{~m}$ has a uniform sound speed and a muddy, lossy bottom. Therefore, we expect a direct path from radiated acoustic noise of the vehicle to the acoustic array. In other underwater environments, sound bounces off the sea surface and bottom boundaries, and sound speed can vary drastically, causing refraction of sound.

Sound speed is commonly calculated as an empirical function of temperature, salinity, and static pressure:

$$
c=1449.2+4.6 T-0.055 T^{2}+0.00029 T^{3}+(1.34-0.01 T)(S-35)+0.016 z,
$$

where $c$ is speed of sound in $\mathrm{m} / \mathrm{s}, T$ is temperature in centigrade, $S$ is salinity in parts per thousand, and $d$ is depth in meters [150]. However, the parameters can vary by season, geographical location, and more. The paths of sound propagation are described by Snell's law

$$
\frac{\cos (\theta)}{c}=\text { const }
$$

such that sound bends towards low sound speed regions. As a result, different propagation paths emerge such as in a surface duct, deep sound channel, or convergence zone [150]. Another important consideration are the surface and bottom boundaries of the ocean. The surface acts as a reflector, and can be modeled as a pressure-release, horizontal boundary. The bottom, in contrast, is lossy, and in some locations in the ocean, possesses complex bathymetry.

When sound travels through the ocean, the signal strength of the source decreases with range due to different loss mechanisms. This decrease is measured as transmission loss, defined as

$$
T L=-10 \log \frac{I(r, z)}{I_{0}}=-20 \log \frac{|p(r, z)|}{\left|p_{0}\right|}[\mathrm{dB} \text { re } 1 \mathrm{~m}] .
$$

The main contributors to transmission loss are geometric spreading, attenuation, and scattering. Geometric spreading loss can be approximated for a point source in an unbounded medium as equally spreading over a sphere surface area (in the near field), $T L=20 \log (r)$, or in a bounded medium as a cylinder (in the far field), $T L=10 \log (r)$. Attenuation of sound in sea water increases with frequency. For example, attenuation is approximately $10^{-3} \mathrm{~dB} / \mathrm{km}$ for $100 \mathrm{~Hz}$, and $1 \mathrm{~dB} / \mathrm{km}$ for $10 \mathrm{kHz}$. Propagation paths can also interact with the seafloor, resulting in transmission loss. Bottom materials- which include clay, silt, 
sand, and gravel - have an attenuation that is three to four orders higher (more lossy) than seawater [150].

With refraction due to the change in sound speed, and interactions with the seafloor and bottom boundaries, sound travels in multiple paths to the receiver. Furthermore, scattering can occur due to bottom roughness and ocean surface movements. The effect of having multiple paths could influence the beamformer results- particularly if tracking the depth of the vehicle with a vertical line array and the direct path, surface bounce, and bottom bounce are all received. The effect of multiple paths also affects the Doppler measurements. All the possible paths and their respective range rates results in a different frequency shift, causing a "Doppler spread" over a bandwidth.

\subsection{Range Limitations of UUV Detection from Mo- tor Noise}

In order to predict the range limitations of detecting a UUV from high-frequency motor noise, we implemented shallow-water simulations with BELLHOP beam tracing software [151]. In this method, we start with the Helmholtz equation (the frequency-domain wave equation):

$$
\nabla^{2} p+\frac{\omega^{2}}{c^{2}(x)} p=-\delta\left(x-x_{s}\right)
$$

where $p$ is pressure, $\omega$ is frequency, $c$ is sound speed, $x_{s}$ is the position of the source. Equation 5.4 is solved with a solution in the form of a ray series, in which the rays are perpendicular to the wavefront. The software can predict the acoustic pressure field by accounting for the sound speed profile, surface and bottom boundaries. For calculating coherent transmission loss, the pressure field at position $(r, z)$ is calculated by summing the pressure for every eigenray $j$ that passes through the point $(r, z)$ :

$$
p^{(C)}(r, z)=\sum_{j=1}^{N(r, z)} p_{j}(r, z),
$$

with $N(r, z)$ as the total number of eigenrays for the range and depth $(r, z), p_{j}(r, z)$ is the pressure for the individual eigenray, $j$. Under some conditions [150], calculating incoherent transmission loss may be sufficient (where the phase of the pressure for each eigenray is 
disregarded):

$$
p^{(I)}(r, z)=\left[\sum_{j=1}^{N(r, z)}\left|p_{j}(r, z)\right|^{2}\right]^{1 / 2} .
$$

One limitation of ray tracing models are the creation of shadow zones where no ray passes. No rays pass through these zones, but in reality, there is a gradual decrease in energy.

In the set-up, we assumed a soft bottom to mimic the Charles River bottom, where the speed of sound is less than water: $1450 \mathrm{~m} / \mathrm{s}$. We also assumed a bottom density of $1200 \mathrm{~kg} / \mathrm{m}^{3}$ and a bottom absorption of $2.0 \mathrm{~dB} / \mathrm{km}$. We used a frequency of $20 \mathrm{kHz}$, and considered two source levels for the thruster and fin noise respectively: $90 \mathrm{~dB}$ and $110 \mathrm{~dB}$. Furthermore, we used the Thorp model for volume attenuation [152]. Using the SONAR equation, we estimated the signal excess received at the hydrophone array as:

$$
S E=S L-T L-N L+A G-D T
$$

where SE is signal excess, SL is the source level from the motor, TL is the transmission loss generated from BELLHOP, NL is approximated as $45 \mathrm{~dB}$ from the field test dataset, AG is the array gain for a six element uniform array, and the detection threshold is set to zero. To illustrate how far a vehicle could be detected under these conditions, the maximum detection range with a detection threshold of zero is reported in the incoherent SE plots. In regards to the noise level, ambient noise in the ocean depends on many factors including sea state and proximity to industrial activity. Generally, ambient noise decreases with an increase in frequency. At $20 \mathrm{kHz}$, ambient noise levels range from $16 \mathrm{~dB}$ to $66 \mathrm{~dB}$ [150]. Three environments were assessed: extreme shallow water (emulating the Charles River) in Figure 5-1, winter shallow water in Figure 5-2, and summer shallow water in Figure 5-3. The seasonal shallow water profiles were adapted from Kuperman et al. [153].

In the extreme shallow-water scenario, the sound speed profile is uniform, as shown in Figure 5-1a. Therefore, in Figure 5-1b, the directions that energy propagates as eigenrays are plotted as straight lines, fanning out from the source. In the incoherent SE plots, in Figures 5-1c-d we show where the Charles River measurement of the Sandshark UUV was taken (100 $\mathrm{m}$ range from vehicle to the array). Because of the difference in source level of the thruster and fin motors of $20 \mathrm{~dB}$, this results in different detection ranges: $734 \mathrm{~m}$ and $2997 \mathrm{~m}$. In the coherent transmission loss plots of Figure 5-1e-f we observe an interference pattern 
due to having a source (the UUV) radiate near a perfect reflector: a smooth, horizontal sea surface.

During winter months, we expect the sound speed profile to be mostly isothermal due to mixing, but also linearly increase with depth as shown in Figure 5-2a. This upward refracting profile causes sound to propagate upwards, bouncing off the surface, which is observed in the eigenrays of Figure 5-2b. In the incoherent SE plots of Figures 5-2c-d, we show that the detection range for the UUV is $504 \mathrm{~m}$ and $2507 \mathrm{~m}$ for thruster and fin motor noise. Because of the upward refracting profile, rays travel near the surface. As a result, the UUV can be detected at further ranges up to $3700 \mathrm{~m}$ if the receiver is closer to the surface as shown in Figure 5-2c. The interference pattern in Figure 5-2f of the coherent SE for fin noise shows rays bending upwards and interfering with surface bounce rays.

As shown in the summer sound speed profile of Figure 5-3a, the top layer has an increase in sound speed, which is due to near-surface heating. Below this layer, the temperature drops and the sound speed decreases. We have modeled this top layer as isothermal. Therefore, in the eigenray plot for the summer profile, in Figure 5-3b, a surface-duct forms and rays are trapped near the surface. Some rays propagate downwards via deep-refracted and surfacebounce paths, where sound speed is slower. As a result, a shadow zone forms where rays do not propagate. This phenomena is illustrated well in Figure 5-3f. In comparison to the winter profile, because of the emergence of a shadow zone, the detection range is shorter: $425 \mathrm{~m}$ and $1414 \mathrm{~m}$ for thruster and fin noise.

The detection ranges from $425 \mathrm{~m}-2997 \mathrm{~m}$ depending on the source level of the motor noise, the sound speed profile, the water depth, and the source and receiver depth.

\subsection{Future Work}

For integrating this method into real passive sonar systems, there are a couple of key research areas for future development: low-frequency motor noise, environmental modeling, 3-D tracking, multi-target tracking, and additional acoustic characterization of the motor design inside the UUV. In this section, improvements to the array signal processing chain are also discussed.

Although the focus of this work was on BLDC motors, electric motors in general are rich with tones that could identify the motor type for UUV classification and the RPM for 
speed estimation. Tones correspond to repetitive discontinuities in the motor design and are a function of propeller rotation. For example, in brushless DC motors, two common acoustic noise sources and their respective tones are: torque ripple $\left(f_{t r}=6 f_{s}\right)$ and cogging torque $\left(f_{c t}=\operatorname{LCM}(s, p) f_{s}\right)$, where $f_{s}$ is the shaft rotation frequency, $s$ is the stator teeth number, $p$ is the pole number, and LCM is the least common multiple. Low frequency tones from the motor could extend the range of this method.

In future implementations of this method, the acoustic array should be optimized for measuring the high-frequency motor tones. Additional elements can be added to the line array to increase the aperture, while the $d$ spacing should be $d<\lambda / 2$ to prevent spatial aliasing. For detecting the UUV from motor noise, this implementation relies on a bandpass filter to isolate the motor acoustic signature. Given a known PWM switching frequency of the target UUV's motor, a phase-locked loop (PLL) could be applied to recover the motor tones in the presence of background noise [154]. The PLL "locks in" on the motor signal at a particular frequency, amplifies the signal of interest, and rejects noise from other frequencies.

With knowledge of the statistical properties of the signal and background noise, adaptive beamforming can improve the bearing estimation of the target UUV. In practice, the number of snapshots $(K)$ for estimating the sample covariance matrix of the signal and noise may be limited. Therefore, techniques such as diagonal loading can be applied [155]. Diagonal loading involves adding a constant diagonal matrix to the sample covariance matrix so it is non-singular for the $K<N$ case ( $N$ is the number of elements).

In order to provide insight into how far a UUV can be detected, the true source level of the motor noise should be characterized, along with its radiation pattern. The true source level of the UUV could be measured by the experimental method described in Holmes et al. where the REMUS class vehicle was secured to a rotating shaft on a barge in a quiet pond environment [119]. Given the true source level, the transmission loss in different ocean environments can be assessed in acoustic propagation models. The modeled transmission loss can also be validated through experimentation. For example, a UUV could travel outand-back from the passive acoustic array. The UUV signature measured from the array could be compared to the range travelled by the robot. To verify the radiation pattern of the robot, additional measurements of the vehicle moving in a circle could be taken. The value of knowing the true source level of the vehicle is that amplitude difference could be 
used as an additional measurement to estimate range. This information can also be used for higher fidelity range detection calculations. Given an accurate source level of the robot, detection ranges in other environmental conditions can be extrapolated, such as deep-water and arctic water propagation. Additional modeling of the Doppler spread due to multi-path propagation should be analyzed. This analysis could inform the robustness of the Kalmanfiltered state estimate to uncertainty in the range rate measurement due to the Doppler spread. Lastly, a known source model of the UUV could be used for matched-field inversion to find the range-averaged sound speed profile as demonstrated in Zhang et al [156].

In this thesis work, we assumed the robot moved in a 2-D plane. An extension of this work is to include estimating the depth of the vehicle. This could be accomplished by adding a vertical line array, and accounting for the expected arrival paths of the UUV source to the array, assuming a known ocean environment. The state estimation technique could also be modified to include depth as a state variable.

One of the assumptions for our tracking demonstrations is a known PWM switching frequency and pole number. Therefore, in a real system, a classifier system should be developed so that the PWM switching frequency and pole number can be identified automatically for a specific robot. Other acoustic features we observed in the motor acoustic signature could be explored for classification too, such as the slot number.

In the experimental results, we showed how passing boats resulted in additional observed tracks in the beamformer results. Therefore, multi-target tracking could be explored to track ships in addition to the robot. The state estimation technique would accordingly have to support multi-target tracking, such as using a particle filter.

A different direction to take this work is to mitigate acoustic noise in UUVs. The measurements taken from the Sandshark and Riptide vehicles can be used as baseline measurements for designing an "undetectable" UUV. The source level of high-frequency motor noise emitting from the Riptide was about 100-110 dB. This platform utilized trapezoidal commutation. Therefore, we can likely attribute its loud tones from torque ripple. The Sandshark thruster, on the other hand, radiated acoustic noise at $80-90 \mathrm{~dB}$. This thruster leveraged sinusoidal commutation which reduces torque ripple. For the Charles River scenario, we approximate an undetectable vehicle source level would need to be approximately $60 \mathrm{~dB}$. This source level goal is $50 \mathrm{~dB}$ quieter than the Riptide (trapezoidal), and $30 \mathrm{~dB}$ quieter than the Sandshark (sinusoidal). To achieve this, we recommend modifying the 
pole design (skewing) and eliminating mechanical resonances near the switching frequency. Furthermore, using a more sophisticated commutation scheme in the motor could achieve lower levels of noise. Field oriented control (FOC), which was not investigated in this thesis work, is a highly efficient commutation scheme that produces low torque ripple by controlling the current phase vector. The acoustic noise reduction due to this commutation scheme should be measured. Open-source designs for implementing FOC control in BLDC motors are available and customizable [157]. In general, reducing acoustic noise in electric motors via the commutation method is an area of active research. For example, Sumega et al. recently demonstrated that implementing FOC control with a Current Harmonic Controller over conventional FOC control significantly reduced acoustic noise in low-cost permanent magnet motors [50]. Understanding and ultimately preventing the transmission paths of the motor vibration to the robot hull and other subsystems should be explored. Vibration of the motor due to PWM current harmonics could be dampened or isolated with this knowledge. Other general design recommendations for improving the motor performance include sensorless methods for reducing cost.

Another area of research to consider is to leverage the motor noise onboard the vehicle. Measuring RPM from the acoustic noise emitted from the motor can serve as a low-cost alternative to an optical encoder. Furthermore, the vibro-acoustic signal can be used to monitor the health of the vehicle's propulsion system, as a fault detector: a change in sound of regular operation could indicate an issue with the motor.

\subsection{Concluding Remarks}

Understanding sources of acoustic noise in UUVs will be an ongoing research field as technology changes and improves for UUVs. With this dissertation work, I hope to inspire future research into characterizing motor noise generally, that could help inform inherit trade-offs of cost, efficiency, and power when selecting the right motor for a quiet UUV design. This thesis provides direct measurements of UUVs that can inform exactly how quiet a UUV needs to be to qualify as undetectable. Furthermore, I hope this research leads to new implementations of using motor noise as a tool for UUV navigation and tracking. The importance of measuring motor acoustic noise is that it provides propeller rotation rate. In this thesis work, I demonstrated the significant advantage of adding an additional mea- 
surement of propeller rotation rate to conventional bearing-Doppler target motion analysis. Motor noise could serve as a low-cost navigation aid to acoustic beacon positioning systems for relative navigation for a swarm of UUVs. Furthermore, the findings of this thesis can be used as a foundation for automatic, real-time UUV detection, classification, localization, and tracking in passive sonar systems of harbors, ports, ships, and submarines. 


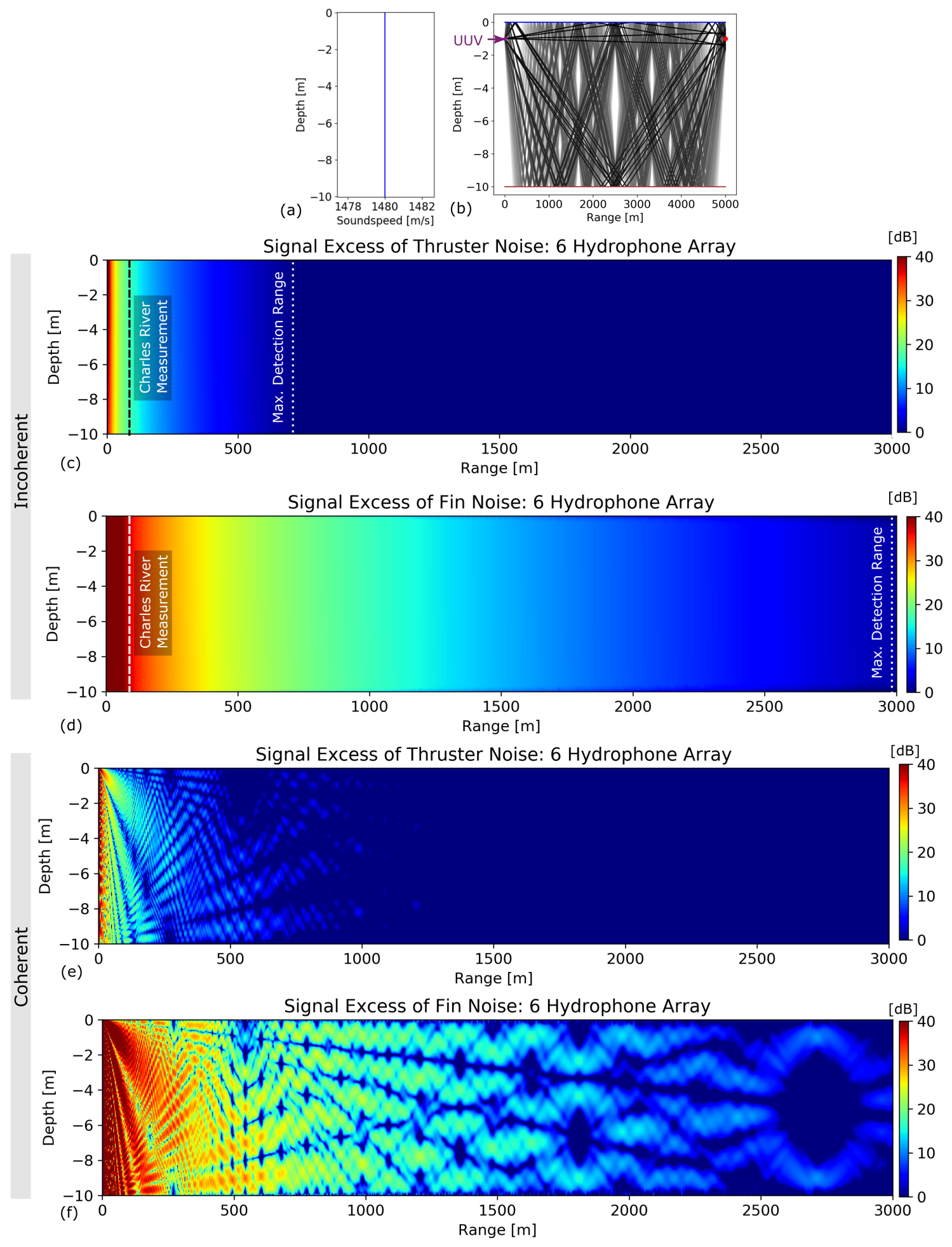

Figure 5-1: Ray tracing results are presented for extreme shallow water of $10 \mathrm{~m}$ depth, where the UUV is at $1 \mathrm{~m}$ depth: (a) sound speed profile, (b) eigenray paths, (c) incoherent and (e) coherent signal excess for thruster noise, and (d) incoherent and (f) coherent signal excess for fin noise 


\section{Winter}
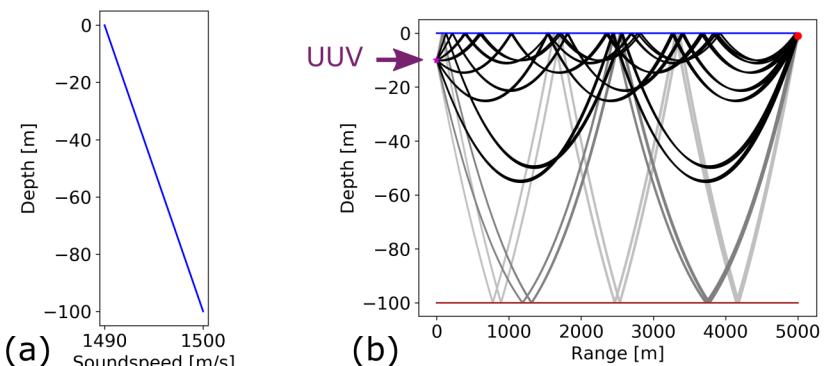

Signal Excess of Thruster Noise: 6 Hydrophone Array

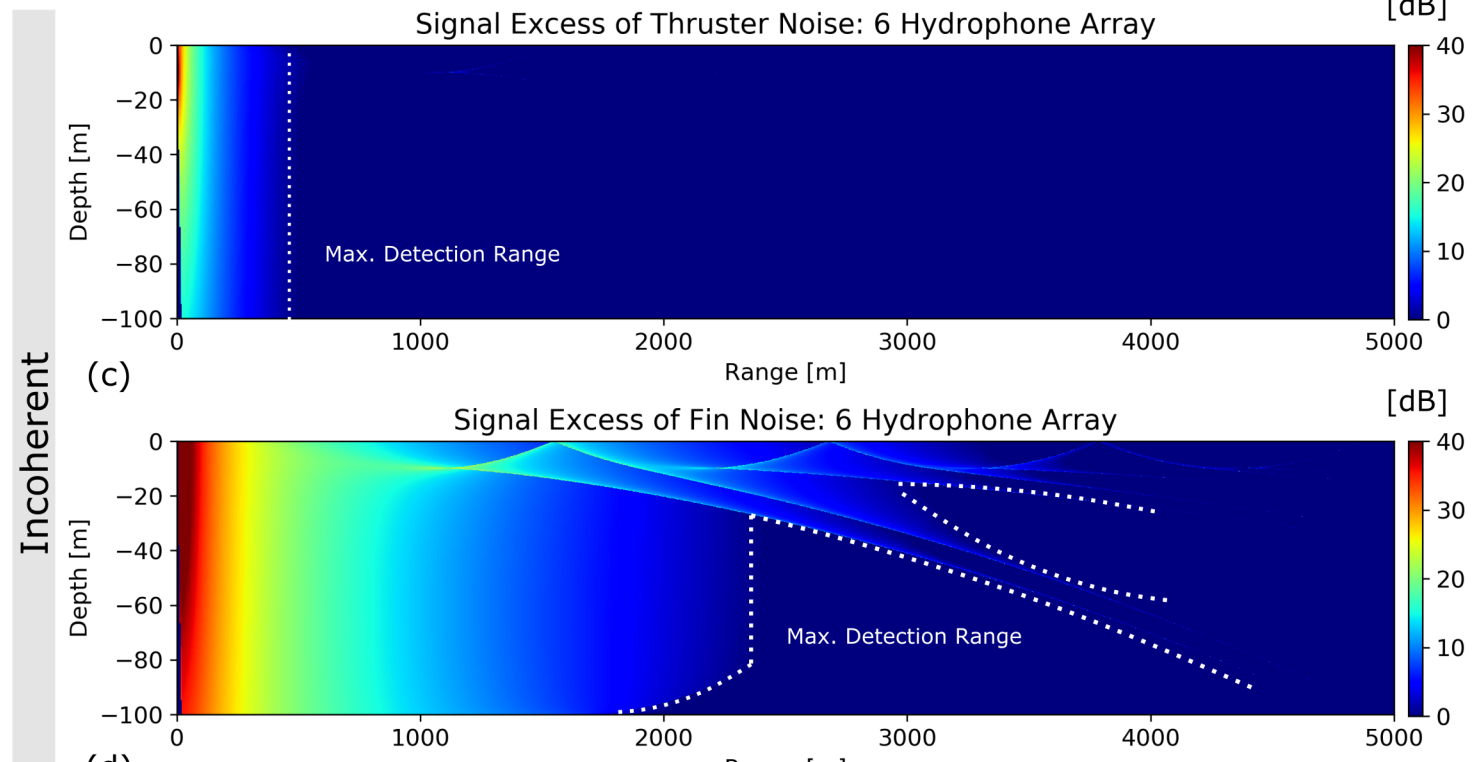

(d)

Range [m]

$[\mathrm{dB}]$

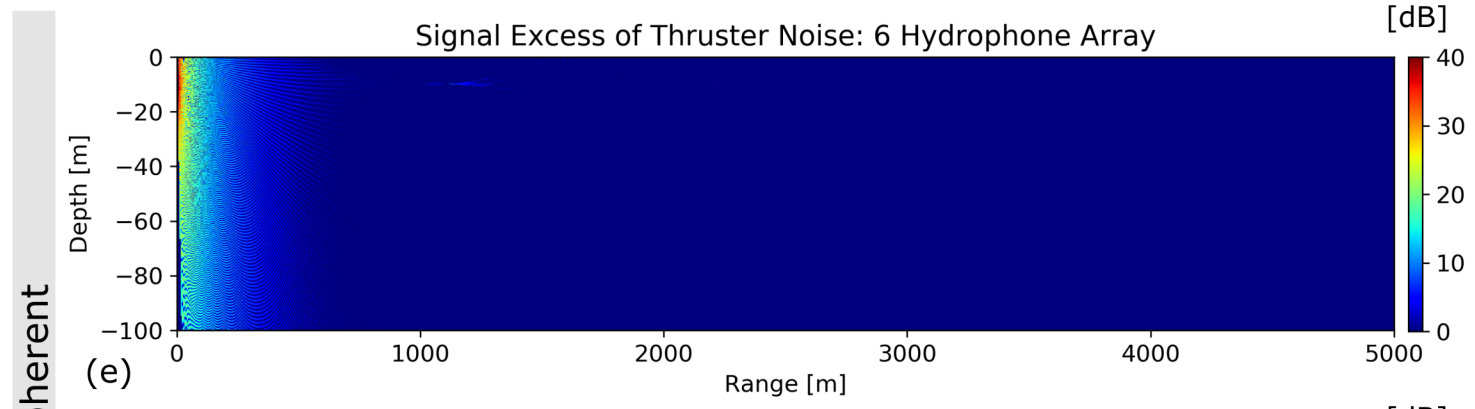

Signal Excess of Fin Noise: 6 Hydrophone Array

[dB]

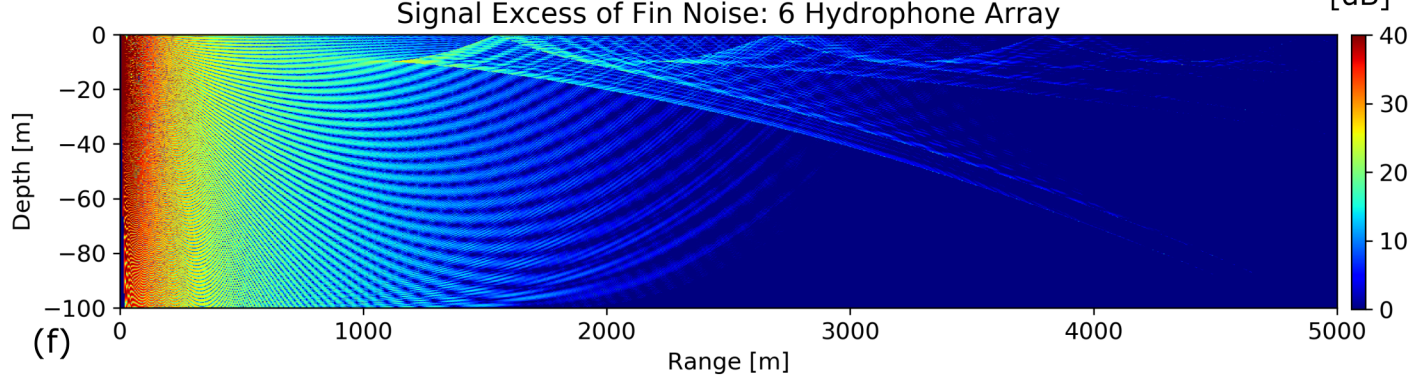

Figure 5-2: Ray tracing results are shown for shallow water in the winter of $100 \mathrm{~m}$ depth, where the UUV is at $10 \mathrm{~m}$ depth: (a) sound speed profile, (b) eigenray paths, (c) incoherent and (e) coherent signal excess for thruster noise, and (d) incoherent and (f) coherent signal excess for fin noise 


\section{Summer}
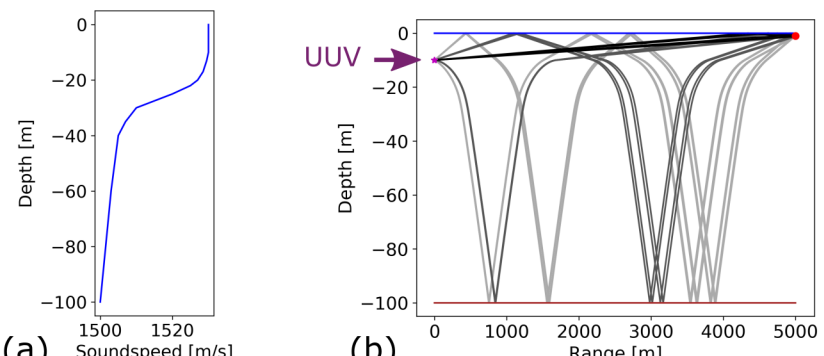

(a) Soundspeed $[\mathrm{m} / \mathrm{s}]$

(b)

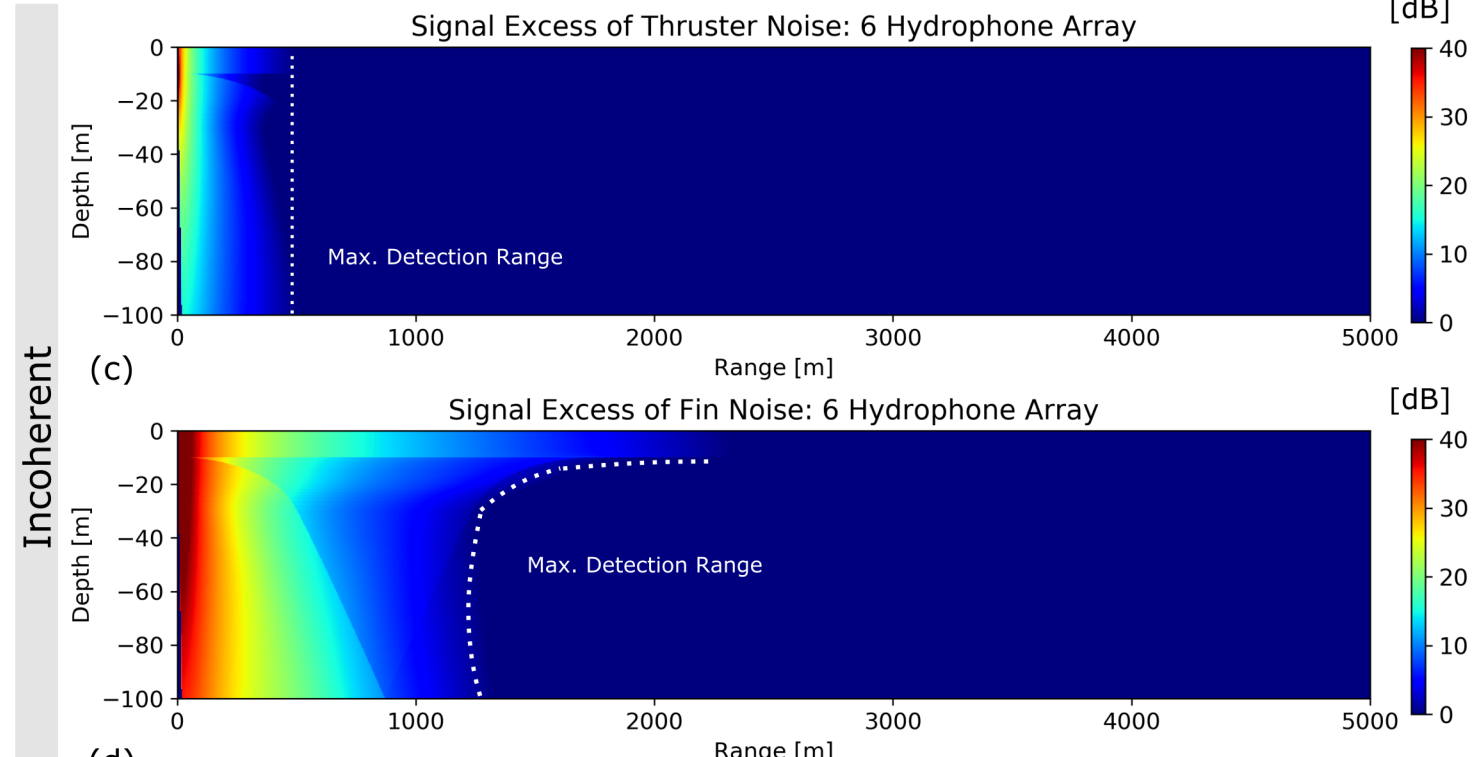

(d)

Range $[\mathrm{m}]$

$[\mathrm{dB}]$

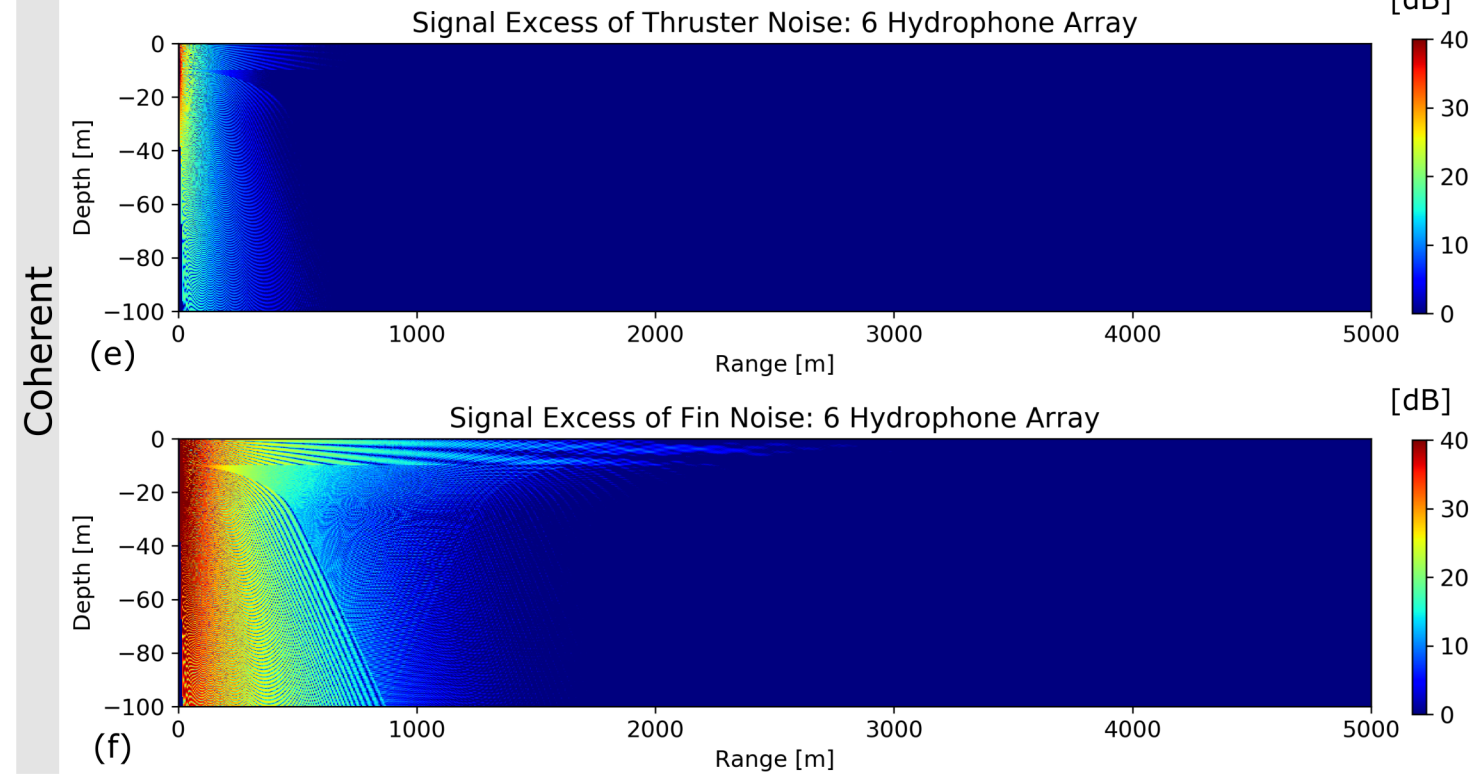

Figure 5-3: Ray tracing results are shown for shallow water in the summer of $100 \mathrm{~m}$ depth, where the UUV is at $10 \mathrm{~m}$ depth: (a) sound speed profile, (b) eigenray paths, (c) incoherent and (e) coherent signal excess for thruster noise, and (d) incoherent and (f) coherent signal excess for fin noise 


\section{Appendix A}

\section{MPDR Beamforming Results}

The conventional beamforming results can be improved by using adaptive beamforming, which accounts for the statistical properties of the signal and background noise. In the field experiment of the Charles River with the Sandshark UUV, we implemented the minimum power distortionless response (MPDR) filter. The sample covariance matrix for the 6element array was calculated from averaging over 6 1-second time-snapshots. Assuming the target UUV's bearing rate is $2^{\circ} / \mathrm{s}$, the aperture length is $0.45 \mathrm{~m}$, and wavelength is $0.074 \mathrm{~m}$, the UUV is in the beam for approximately 5 seconds. Figures A-1a-d are a comparison of conventional and adaptive beamforming on the broadband noise of the vehicle (1000$8000 \mathrm{~Hz}$ ). Figures A-2a-d are a comparison of conventional and adaptive beamforming on motor noise $(17450-17550 \mathrm{~Hz})$. As shown in the direction of arrival plots, Figures A-1b,d and Figures A-2b,d the beamwidth narrows when applying MPDR. 

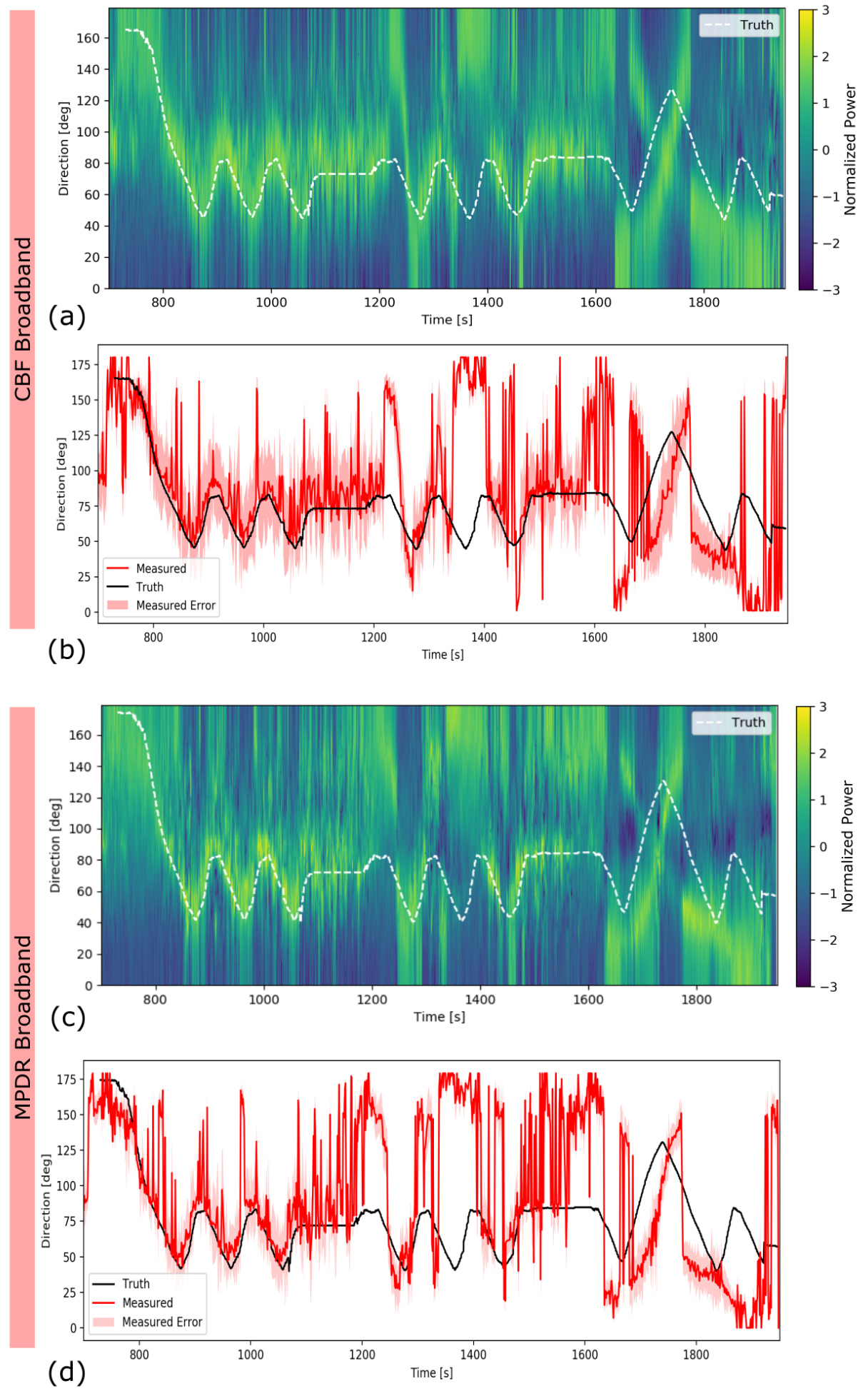

Figure A-1: Comparison of applying (a) conventional beamforming and (c) MPDR beamforming to the broadband noise from the Sandshark UUV. The respective DOA plots are in (b) and (d) 

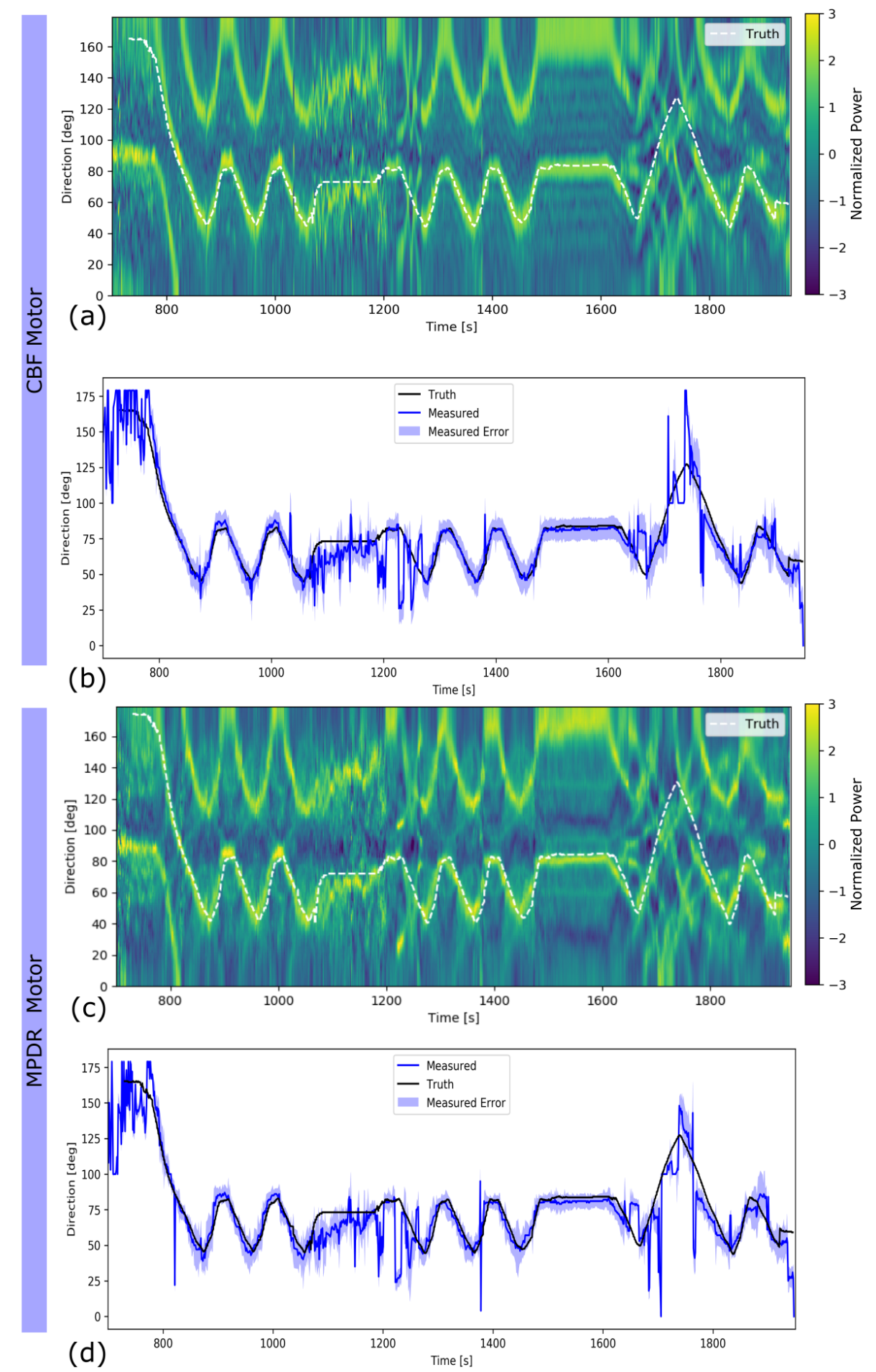

Figure A-2: Comparison of applying (a) conventional beamforming and (c) MPDR beamforming to the motor noise from the Sandshark UUV. The respective DOA plots are in (b) and (d) 


\section{Appendix B}

\section{UUV Source Level Estimates and Additional DEMON Algorithm Analysis}

Power spectral density (PSD) estimates for each robot demonstrate how quiet cavitation noise is for a UUV. Figures B-1a-d are the PSD plots for the Sandshark UUV at low (400 rpm) and high speeds (1000 rpm). As shown in Figure B-1b and Figure B-1d, when the robot rotates its propeller at higher speeds, the overall broadband noise of the robot increases. However, the increase in broadband noise is only $5-10 \mathrm{~dB}$ higher than the background of the pool environment. In contrast, the motor acoustic signature in the thruster provides high SNR measurements of $30 \mathrm{~dB}$ above the ambient noise. The power spectral density of the Sandshark UUV fin movement is plotted in Figure B-2. The fins emit significant broadband noise as well as loud tones at the PWM switching frequency, as shown in Figure B-2. An estimate of the source level of the Sandshark's thruster PWM switching frequency is $78 \mathrm{~dB}$ and the estimated source level of the fin PWM switching frequency is $101 \mathrm{~dB}$.

The Riptide UUV was an overall louder platform, as shown in the PSD plots from the pool experiment in Figures B-3. A high and low speed of the vehicle are compared to show how the noise of the vehicle increases with RPM. The source level of the motor PWM switching frequency was $103 \mathrm{~dB}$.

The DEMON algorithm performance is highly dependent on the frequency range of the 

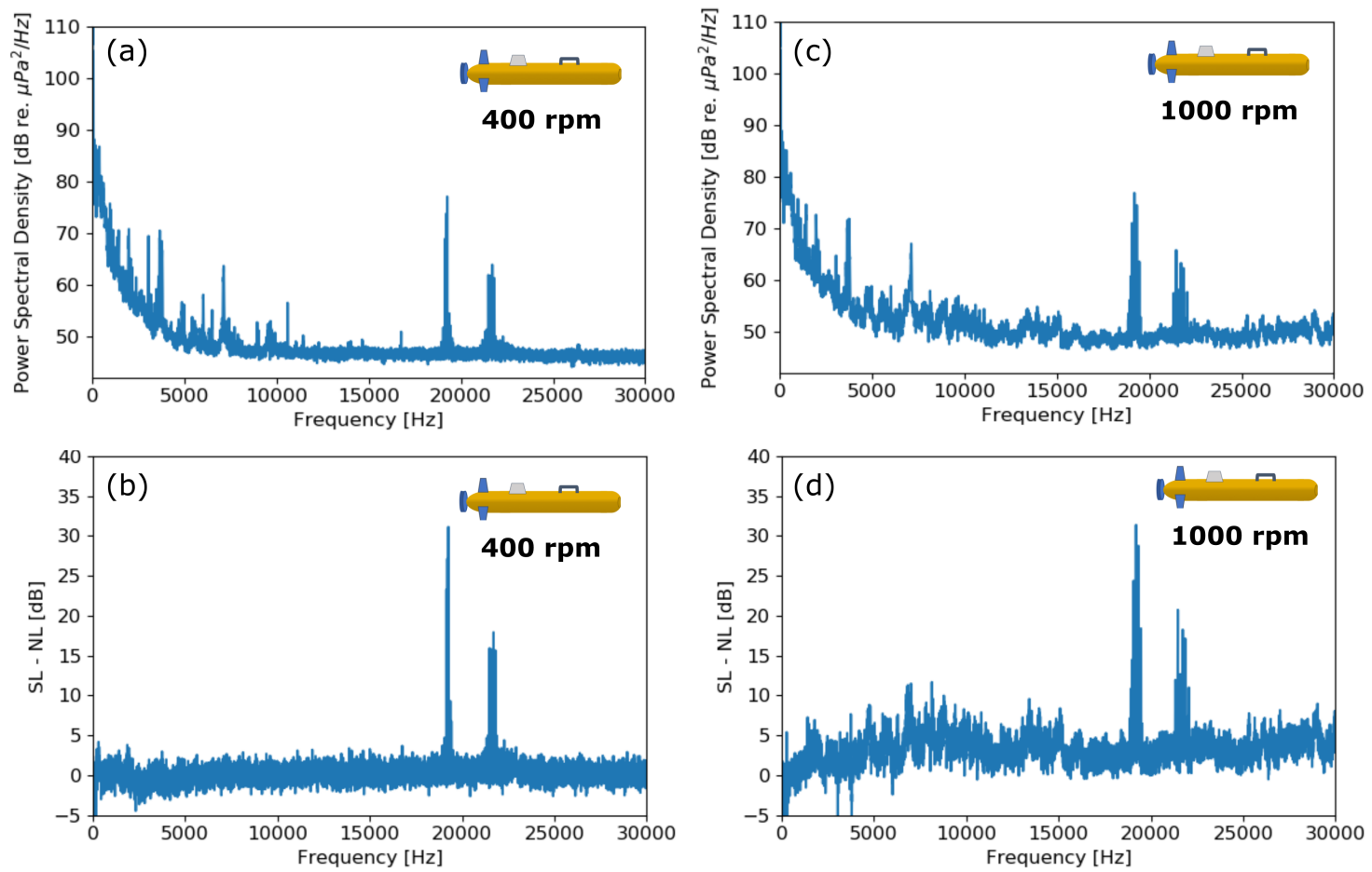

Figure B-1: Acoustic data was collected on the radiated noise of the Sandshark UUV in a pool at a high and low speed to estimate the robot's acoustic signature. (a) Power spectral density of the Sandshark UUV at $400 \mathrm{rpm}$. (b) Source level of the UUV above the ambient noise. (c) Power spectral density of the Sandshark UUV at $1000 \mathrm{rpm}$. (d) Source level of the UUV at $1000 \mathrm{rpm}$ above the ambient noise.

bandpass filter. Therefore, in this section, we evaluate different bandpass filters on the pool and field experiments, for the Sandshark and Riptide vehicles. While the purpose of the DEMON algorithm is to isolate broadband cavitation noise, we also applied the DEMON algorithm to the motor noise. The motor switching frequency is a carrier with sidebands at harmonics of the fundamental shaft rotation frequency.

Two filters are compared for the Sandshark pool test in Figure B-4: $10 \mathrm{kHz}-18.88 \mathrm{kHz}$ (broadband noise) and $19 \mathrm{kHz}-19.2 \mathrm{kHz}$ (motor noise). Tones at the shaft rotation frequency times the pole number dominate in both Figures B-4a-b.

For the Riptide pool test, low frequency tones were observed. Therefore, in choosing a filter for applying the DEMON algorithm to this vehicle, we applied two bandpass filters to capture broadband cavitation noise: $1 \mathrm{kHz}-17 \mathrm{kHz}$ and $10 \mathrm{kHz}-17 \mathrm{kHz}$, shown in Figures B5a-b. Then, to isolate the motor signature we also considered the frequency bandpass filter of $17.2 \mathrm{kHz}-18.6 \mathrm{kHz}$ in Figure B-5c. 
For the Riptide Charles River field test, we applied three different bandpass filters to calculate the DEMON spectrum, shown in Figure B-7. Considering broadband noise from $1 \mathrm{kHz}-17 \mathrm{kHz}$ in Figure B-7a, characteristic tones from the cavitation noise are not observable. By moving to a higher frequency range at $10 \mathrm{kHz}-17 \mathrm{kHz}$ in Figure $\mathrm{B}-7 \mathrm{~b}$, tones at the poles times the shaft rotation frequency are present when the vehicle is in close range (285-300 s). In contrast, the measured signal of the motor noise in Figure B-7c is observable during the whole mission. For the Sandshark Charles River test, two filters for the DEMON algorithm are evaluated: $10 \mathrm{kHz}-17.4 \mathrm{kHz}$ in Figure B-7a (cavitation noise) and $18.1 \mathrm{kHz}-$ $18.4 \mathrm{kHz}$ in Figure B-7b (motor noise). Again, the tones from the cavitation noise of the UUV are not observable. However, as shown in Figure B-7b, tones from the motor noise are present when the vehicle is underway (such as the segment from $800-1000 \mathrm{~s}$ ).

Ideally, in a quiet environment, one would have both motor noise and the DEMON spectrum of cavitation noise to determine propeller rotation and other critical characteristics. However, as shown in the Charles River field tests, the DEMON spectrum is challenging to measure in a harbor-like environment. 

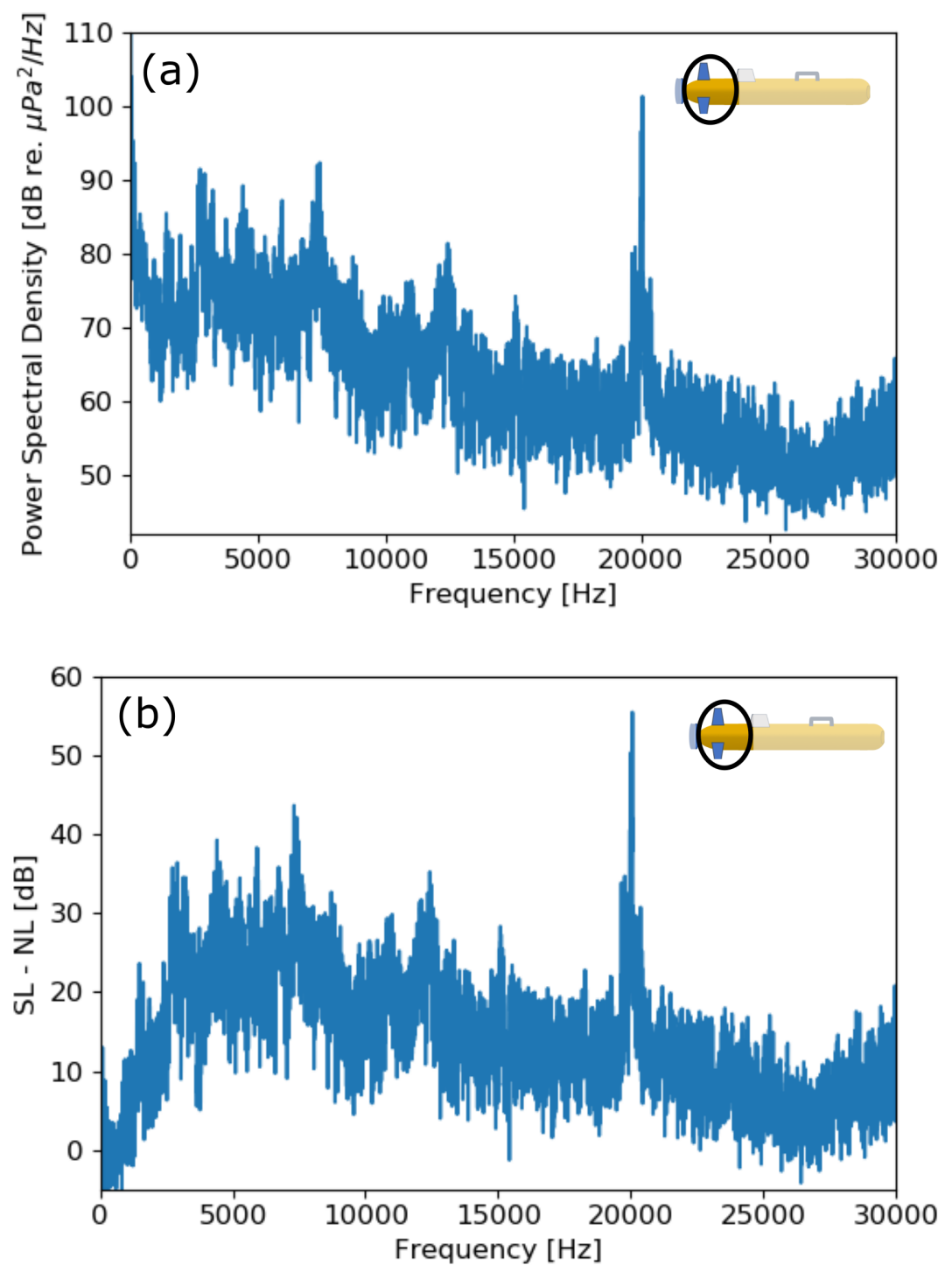

Figure B-2: Acoustic data was collected on the radiated noise of the Sandshark UUV fins in a pool (a) Power spectral density of the fins rotating. (b) Source level of the UUV fins above the ambient noise. 

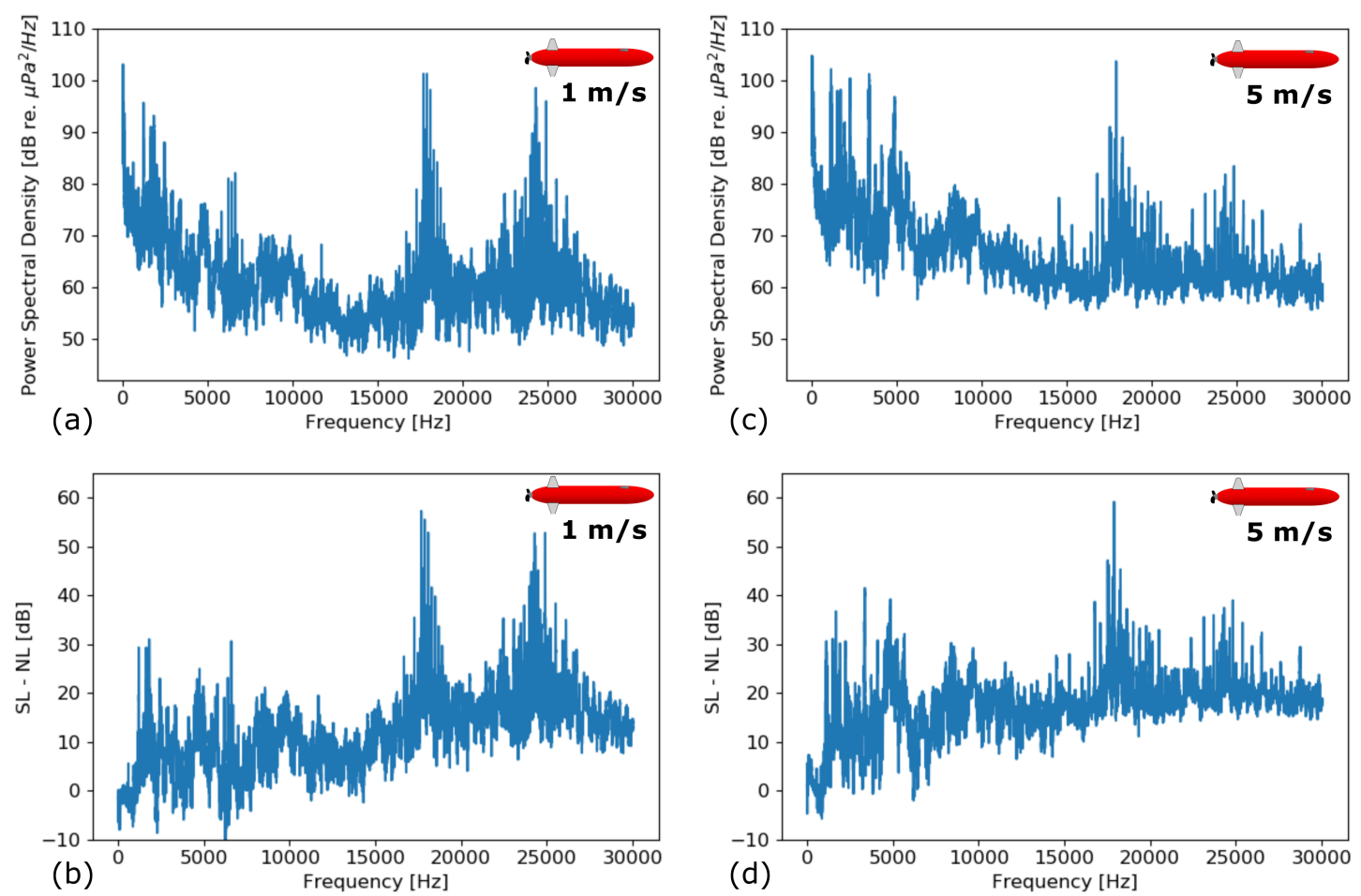

Figure B-3: Acoustic data was collected on the radiated noise of the Riptide UUV in a pool. (a) Power spectral density of the robot with a desired speed of $1 \mathrm{~m} / \mathrm{s}$ (b) Source level of the UUV above the ambient noise at a desired speed of $1 \mathrm{~m} / \mathrm{s}$. (c) Power spectral density of the robot with a desired speed of $5 \mathrm{~m} / \mathrm{s}$ (d) Source level of the UUV above the ambient noise at a desired speed of $5 \mathrm{~m} / \mathrm{s}$.
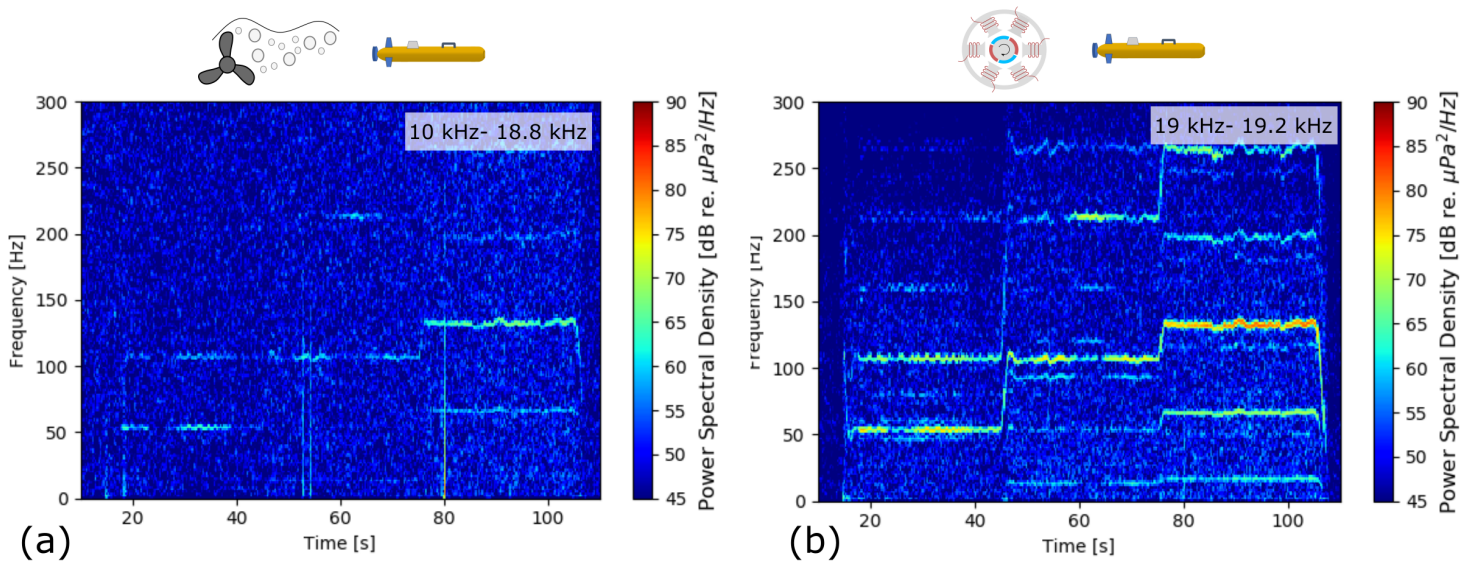

Figure B-4: Comparison of applying the DEMON algorithm to the Sandshark UUV pool test data for (a) broadband noise and (b) high-frequency motor noise 


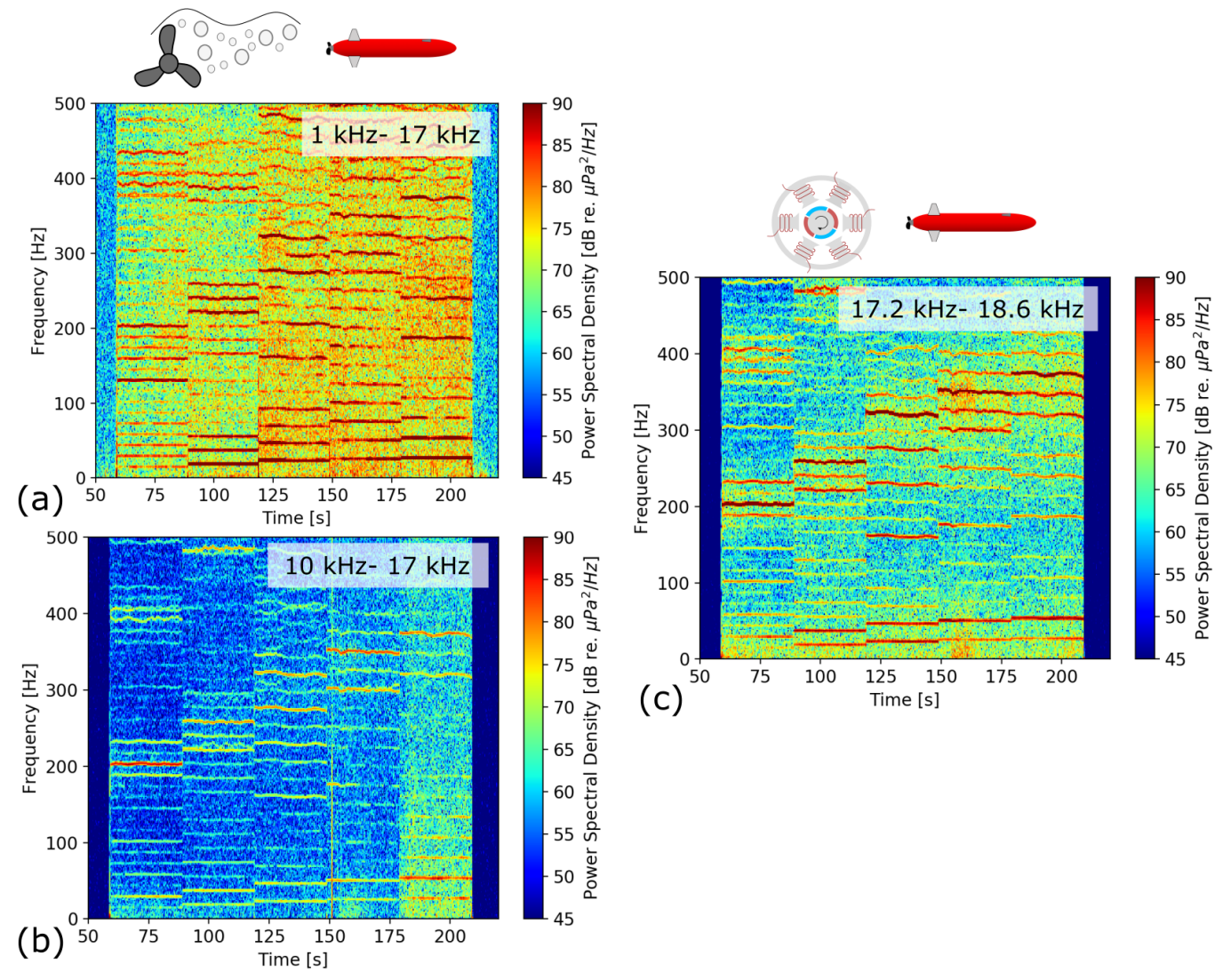

Figure B-5: Comparison of applying the DEMON algorithm to the Riptide UUV pool test data for (a) broadband noise from $1 \mathrm{kHz}-17 \mathrm{kHz}$, (b) broadband noise from $10 \mathrm{kHz}-17 \mathrm{kHz}$, and (c) high-frequency motor noise. 

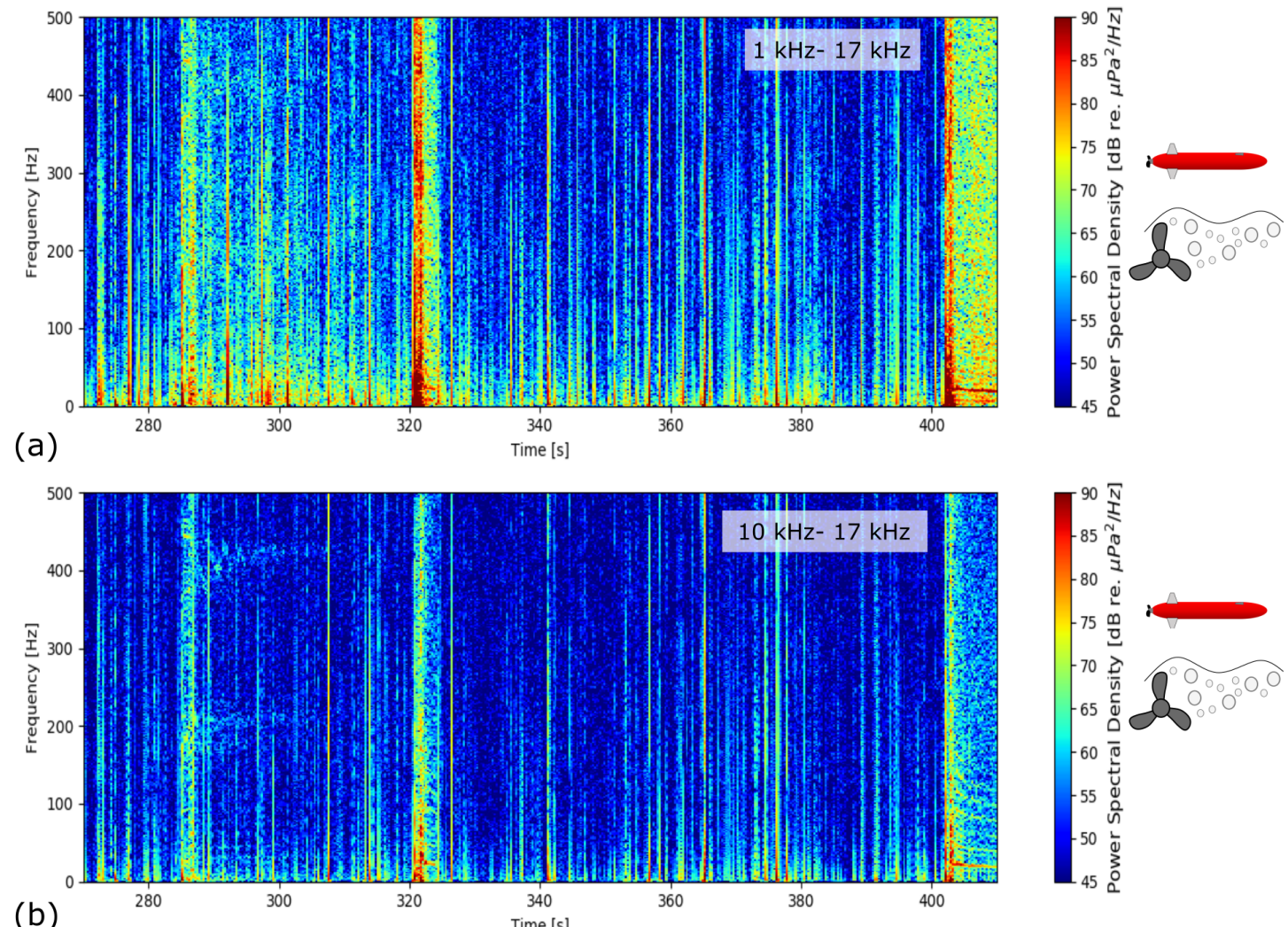

(b)

Time [s]

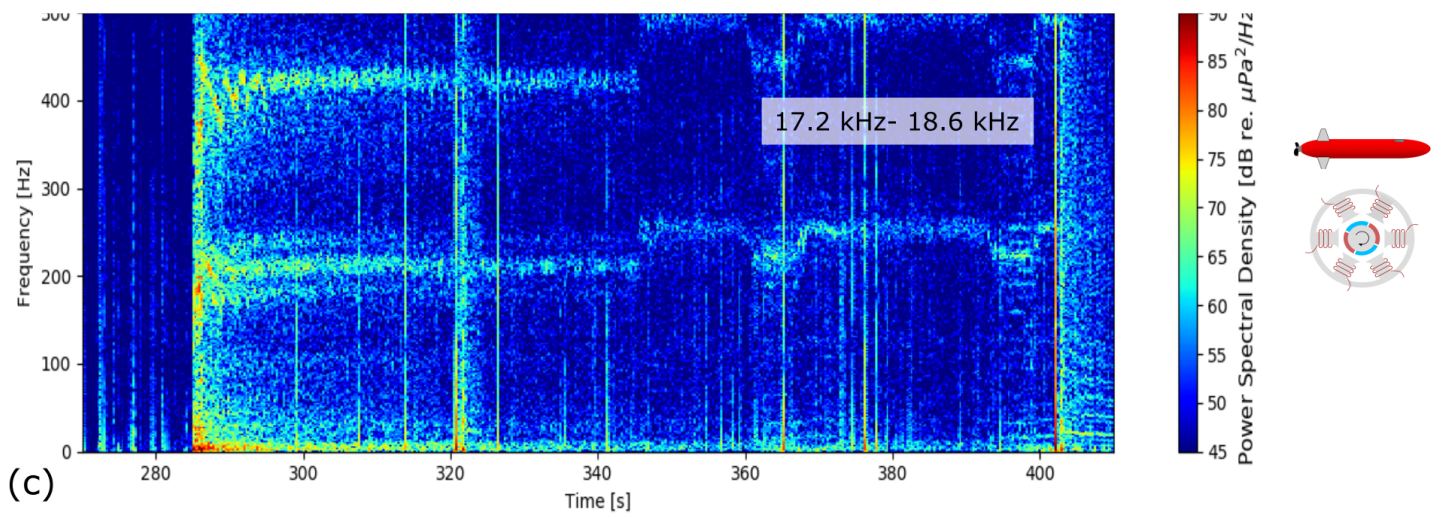

Figure B-6: Comparison of applying the DEMON algorithm to the Riptide Charles River test for (a) broadband noise from $1 \mathrm{kHz}-17 \mathrm{kHz}$, (b) broadband noise from $10 \mathrm{kHz}-17 \mathrm{kHz}$, and (c) high-frequency motor noise 

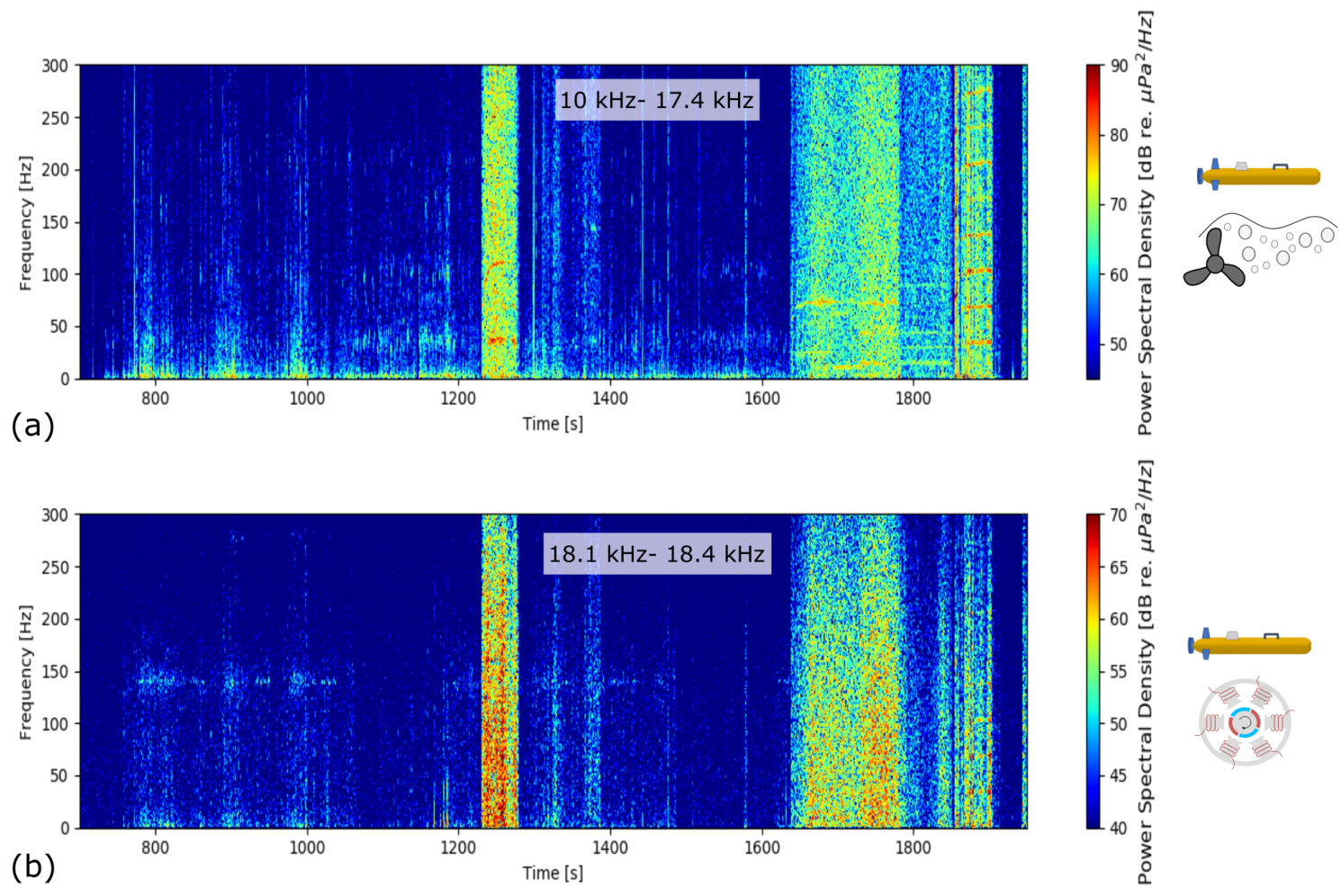

Figure B-7: Comparison of applying the DEMON algorithm to the Sandshark Charles River test for (a) broadband noise from $10 \mathrm{kHz}-17.45 \mathrm{kHz}$ and (b) high-frequency motor noise 


\section{Appendix $\mathrm{C}$}

\section{Observability of Range}

The UUV tracking solution can be uniquely determined from passive sonar measurements under the condition that bearing rate is nonzero and the target moves at a constant speed. To illustrate this, three scenarios are described in Figures C-1-C-3. It should be noted that the convergence of the state estimation technique also depends on the initial conditions. In this thesis work, the unscented Kalman filter results were initialized with ground truth. Future work should investigate the stability of the tracking solution with uncertainty in the initial state.

When the UUV directly approaches or leaves the acoustic array, the bearing is constant. Therefore, bearing rate is zero and range rate equals the speed of the robot. In this scenario, the range of the UUV to the array is ambiguous. As noted in Figure C-1, given the same measurements of bearing, bearing rate, range rate, and speed, the robot could be at any distance along the bearing line.

A unique range can be derived from the passive array measurements- bearing, bearing rate, range rate, and speed- if the target changes bearing. In Figure $\mathrm{C}-2$, the vehicle maintains a constant range to the array, while changing bearing. In this case, the range rate is zero, and consequently, the measured speed is equal to the tangential speed (the arc distance over time). Given bearing rate, there can only be one range solution for the measured speed.

Likewise, in Figure C-3, a unique range solution can be derived when the vehicle changes both bearing and range. The key is that given speed $|\vec{v}|$ and range rate $v_{r}$, the tangential velocity $v_{\theta}$ can be estimated. The tangential velocity is $v_{\theta}=\Delta s / \Delta T$ where $\Delta s$ is the arc 


\section{Ambiguous Range with Zero Bearing Rate (Constant Bearing)}

(a)

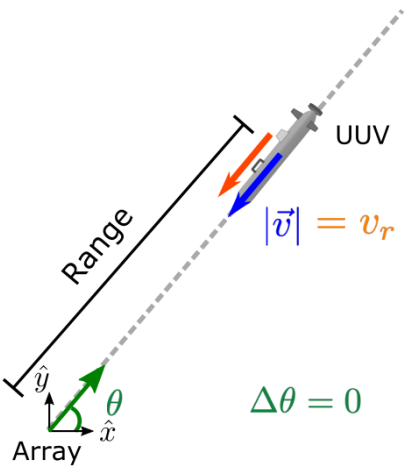

(b)

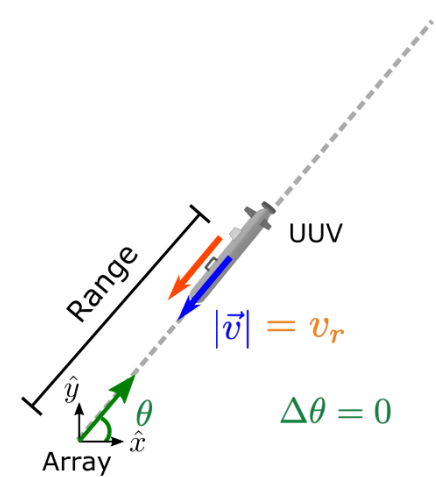

Legend for Measurements

Range Rate: $v_{r} \quad$ Bearing: $\theta$

Speed: $|\vec{v}| \quad$ Change in Bearing: $\Delta \theta$

Figure C-1: Schematic shows the passive sonar measurements for a UUV approaching an array with constant bearing. The same measurements are produced for (a) and (b) which have different ranges.

length travelled by the UUV. With the change in bearing $\Delta \theta$ from bearing rate $\dot{\theta}$, the range $r$ can be estimated:

$$
\begin{array}{r}
r \Delta \theta=\Delta s \\
r \frac{\theta}{\Delta T}=\frac{\Delta s}{\Delta T} . \\
r \dot{\theta}=v_{\theta}
\end{array}
$$




\section{Unique Range with Zero Range Rate (Constant Range)}

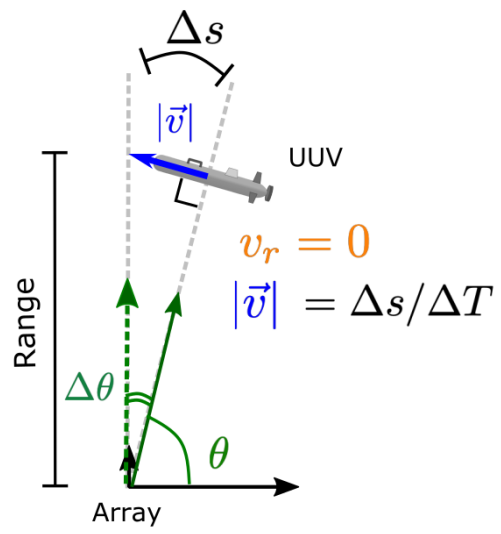

\begin{tabular}{cc} 
Legend for Measurements \\
\hline Range Rate: $v_{r}$ & Bearing: $\theta$ \\
Speed: $|\vec{v}|$ & Change in Bearing: $\Delta \theta$ \\
Arc Length: $\Delta s$ & Change in Time: $\Delta T$
\end{tabular}

Figure C-2: Schematic shows the passive sonar measurements for a UUV moving around an array at a constant range. A unique solution for range is derived for these measurements.

\section{Unique Range with Non-Zero Range Rate and Bearing Rate}

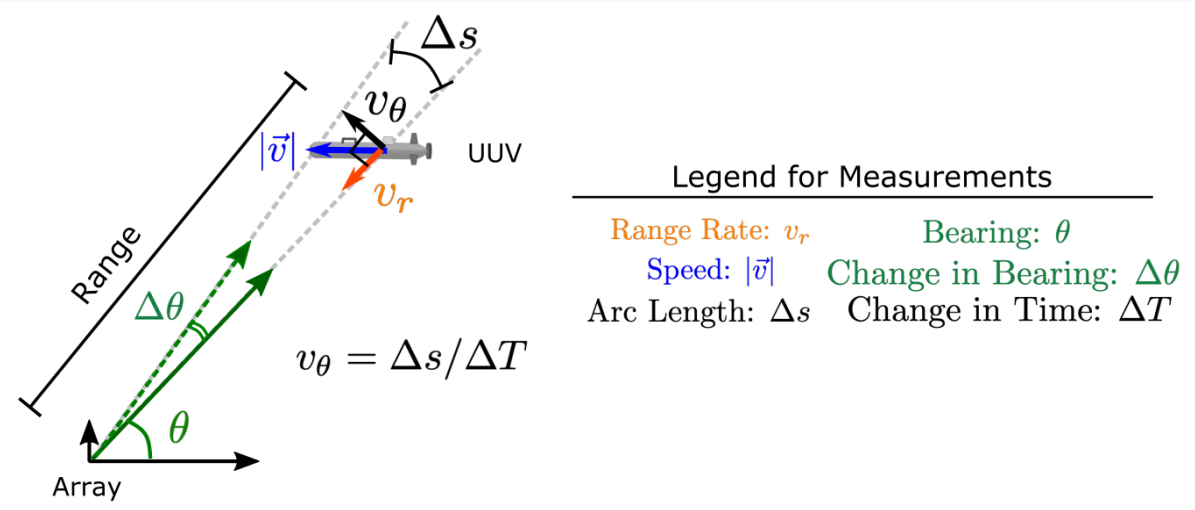

Figure C-3: Schematic shows the passive sonar measurements for a UUV moving with a nonzero bearing rate and range rate. A unique solution for range is derived for these measurements. 


\section{Bibliography}

[1] Robert J. Urick, Principles of Underwater Sound, vol. 3rd edn. McGraw-Hill, 1983.

[2] Y. Ji, B. Li, and J. Sun, "Harmonic Analysis on Torque Ripple of Brushless DC Motor Based on Advanced Commutation Control," Journal of Control Science and Engineering, vol. 2018, 2018.

[3] Y. Bar-Shalom, X. R. Li, and T. Kirubarajan, Estimation with applications to tracking and navigation: theory algorithms and software. John Wiley \& Sons, 2001.

[4] B. Hiremath, IHS Jane's Unmanned Maritime Vehicles. 2015.

[5] G. Griffiths, Technology and Applications of Autonomous Underwater Vehicles. London: Taylor \& Francis, 2003.

[6] "REMUS and Mine Countermeasures." Office of Naval Research. https://www.onr.navy.mil/en/About-ONR/History/tales-of-discovery/remus Accessed:2018-04-25.

[7] D. Clegg and M. Peterson, "User operational evaluation system of unmanned underwater vehicles for very shallow water mine countermeasures," Oceans 2003: Celebrating the Past... Teaming Toward the Future, vol. 3, pp. 1417-1423, 2003.

[8] "REMUS 100." Woods Hole Oceanographic Institution. https://www2.whoi.edu/site/osl/vehicles/remus-100/ Accessed: 2021-09-28.

[9] "REMUS 600." Woods Hole Oceanographic Institution. https://www2.whoi.edu/site/osl/vehicles/remus-600/ Accessed: 2021-09-28.

[10] U.S. Navy, "The Navy Unmanned Undersea Vehicle (UUV) Master Plan," 2004.

[11] S. Neuman, "Failed MH370 Search May Have Solved Mystery Of 19th Century Shipwrecks," National Public Radio, 2018.

[12] P. K. Le Hardy and C. Moore, "Deep ocean search for Malaysia airlines flight 370," 2014 Oceans - St. John's, OCEANS 2014, pp. 31-34, 2015.

[13] R. B. Wynn, V. A. Huvenne, T. P. Le Bas, B. J. Murton, D. P. Connelly, B. J. Bett, H. A. Ruhl, K. J. Morris, J. Peakall, D. R. Parsons, E. J. Sumner, S. E. Darby, R. M. Dorrell, and J. E. Hunt, "Autonomous Underwater Vehicles (AUVs): Their past, present and future contributions to the advancement of marine geoscience," Marine Geology, vol. 352, pp. 451-468, 2014. 
[14] A. J. Poulsen and H. Schmidt, "Acoustic noise properties in the rapidly changing arctic ocean," in Proceedings of Meetings on Acoustics, vol. 28, pp. 1-10, Acoustical Society of America, 2017.

[15] D. Wang, P. F. Lermusiaux, P. J. Haley, D. Eickstedt, W. G. Leslie, and H. Schmidt, "Acoustically focused adaptive sampling and on-board routing for marine rapid environmental assessment," Journal of Marine Systems, vol. 78, pp. S393-S407, 2009.

[16] G. E. Packard, A. Kukulya, T. Austin, M. Dennett, R. Littlefield, G. Packard, M. Purcell, R. Stokey, and G. Skomal, "Continuous autonomous tracking and imaging of white sharks and basking sharks using a REMUS-100 AUV," OCEANS 2013 MTS/IEEE - San Diego: An Ocean in Common, pp. 4-8, 2013.

[17] K. L. Dodge, A. L. Kukulya, E. Burke, and M. F. Baumgartner, "TurtleCam: A Smart Autonomous Underwater Vehicle for Investigating Behaviors and Habitats of Sea Turtles," Frontiers in Marine Science, 2018.

[18] D. R. Yoerger, M. Curran, J. Fujii, C. R. German, D. Gomez-Ibanez, A. F. Govindarajan, J. C. Howland, J. K. Llopiz, P. H. Wiebe, B. W. Hobson, K. Katija, M. Risi, B. H. Robison, C. J. Wilkinson, S. M. Rock, and J. A. Breier, "Mesobot: An Autonomous Underwater Vehicle for Tracking and Sampling Midwater Targets," AUV 2018 - 2018 IEEE/OES Autonomous Underwater Vehicle Workshop, Proceedings, 2018.

[19] A. Martin, P. Boyd, K. Buesseler, I. Cetinic, H. Claustre, S. Giering, S. Henson, X. Irigoien, I. Kriest, L. Memery, et al., "Study the twilight zone before it is too late," Nature, vol. 580, pp. 26-28, 2020.

[20] R. O'Rourke, "Navy Large Unmanned Surface and Undersea Vehicles: Background and Issues for Congress," Congressional Research Service, 2020.

[21] "Department of the Navy Unmanned Campaign Framework," 2021.

[22] "An analysis of the Navy's fiscal year 2022 shipbuilding plan," Congressional Budget Office, 2021.

[23] R. Mabus, "Autonomous Undersea Vehicle Requirement for 2025," 2016.

[24] DARPA, "Special Notice DARPA-SN-16-11 1: Open Ocean Counter Unmanned Underwater Vehicle (OOCUUV) Study," pp. 1-7, 2016.

[25] "Unmanned Undersea Vehicle (UUV) Detection and Classification in Harbor Environments," tech. rep., Office of Naval Research (ONR). Small Business Innovation Research (SBIR) Proposal Submission, 2015.

[26] "Counter-Unmanned Undersea Vehicle (C-UUV) Hosted by the Stiletto Maritime Demonstration Program," tech. rep., ASD Rapid Reaction Technology Office, Stiletto Maritime Demonstration Platform.

[27] D. Wu and S. D. Pekarek, "Using mechanical vibration to estimate rotor speed in induction motor drives," PESC Record - IEEE Annual Power Electronics Specialists Conference, pp. 2412-2417, 2007. 
[28] J. J. Leonard and A. Bahr, "Autonomous underwater vehicle navigation," in Springer Handbook of Ocean Engineering, pp. 341-357, 2016.

[29] D. Thomson, "True Dual Redundant Acoustic Dynamic Positioning Sensors," Dynamic Positioning Committee.

[30] K. Vickery, "Acoustic positioning systems - a practical overview of current systems," Proceedings of the IEEE Symposium on Autonomous Underwater Vehicle Technology, pp. 5-17, 1998.

[31] L. Paull, S. Saeedi, M. Seto, and H. Li, "AUV navigation and localization: A review," IEEE Journal of Oceanic Engineering, vol. 39, no. 1, pp. 131-149, 2014.

[32] A. Mallios, P. Ridao, D. Ribas, and S. Zandara, "Sonar-based simultaneous localization and mapping for autonomous underwater vehicles," in Further Advances in Unmanned Marine Vehicles, pp. 149-170, 2012.

[33] J. Bellingham, "Autonomous Underwater Vehicles (auvs)," in Encyclopedia of Ocean Sciences, pp. 212-216, 2001.

[34] "A Survey of Missions for Unmanned Undersea Vehicle," tech. rep., RAND Corporation, Santa Monica, 2009.

[35] W. A. Salah, D. Ishak, and K. J. Hammadi, "Minimization of torque ripples in BLDC motors due to phase commutation - A review," Przeglad Elektrotechniczny, vol. 87, no. 1, pp. 183-188, 2011.

[36] S.-H. Kim, "Brushless direct current motors," in Electric Motor Control, pp. 389-416, Elsevier, 2017.

[37] "Advanced Development Micro-Unmanned Undersea Vehicle," Riptide Autonomous Solutions.

[38] A. Underwood and C. Murphy, "Design of a micro-AUV for autonomy development and multi-vehicle systems," OCEANS 2017 - Aberdeen, pp. 1-6, 2017.

[39] S. Petillo, H. Schmidt, and A. Balasuriya, "Constructing a distributed AUV network for underwater plume-tracking operations," International Journal of Distributed Sensor Networks, vol. 2012, 2012.

[40] P. S. A. Randeni, N. R. Rypkema, E. M. Fischell, A. L. Forrest, M. R. Benjamin, and H. Schmidt, "Implementation of a Hydrodynamic Model-Based Navigation System for a Low-Cost AUV Fleet," AUV 2018 - 2018 IEEE/OES Autonomous Underwater Vehicle Workshop, Proceedings, 2018.

[41] N. R. Rypkema, Underwater and out of sight: Towards ubiquity in underwater robotics. PhD thesis, Massachusetts Institute of Technology, 2019.

[42] M. R. Benjamin, J. J. Leonard, H. Schmidt, and P. M. Newman, "Nested Autonomy for Unmanned Marine Vehicles with MOOS-IvP," Journal of Field Robotics, vol. 29, no. 4 , pp. 554-575, 2015. 
[43] M. H.A. d'Assumpcao, "Theoretical Assessment of "DEMON" Performance," Department of Supply Australian Defense Scientific Service Weapons Research Establishment, no. Technical Memorandum CPD 169.

[44] K. W. Chung, A. Sutin, A. Sedunov, and M. Bruno, "DEMON acoustic ship signature measurements in an urban harbor," Advances in Acoustics and Vibration, vol. 2011, no. i, 2011.

[45] A. Pollara, A. Sutin, and H. Salloum, "Improvement of the Detection of Envelope Modulation on Noise (DEMON) and its application to small boats," OCEANS 2016 MTS/IEEE Monterey, OCE 2016, 2016.

[46] A. Pollara, A. Sutin, and H. Salloum, "Passive acoustic methods of small boat detection, tracking and classification," 2017 IEEE International Symposium on Technologies for Homeland Security, 2017.

[47] P. Leaders, R. Wynn, and E. Linley, "Global Inventory of AUV and Glider Technology available for Routine Marine Surveying," no. September, pp. 1-153, 2013.

[48] K. Railey, D. DiBiaso, and H. Schmidt, "An acoustic remote sensing method for highprecision propeller rotation and speed estimation of unmanned underwater vehicles," The Journal of the Acoustical Society of America, vol. 148, no. 6, pp. 3942-3950, 2020.

[49] G. Griffiths, P. Enoch, and N. W. Millard, "On the radiated noise of the Autosub autonomous underwater vehicle," ICES Journal of Marine Science, vol. 58, no. 6, pp. 1195-1200, 2001.

[50] M. Sumega, P. Rafajdus, and M. Stulrajter, "Current harmonics controller for reduction of acoustic noise, vibrations and torque ripple caused by cogging torque in PM motors under FOC operation," Energies, vol. 13, no. 10, 2020.

[51] T. M. Jahns and W. L. Soong, "Pulsating torque minimization techniques for permanent magnet AC motor drives - A review," IEEE Transactions on Industrial Electronics, vol. 43, no. 2, pp. 321-330, 1996.

[52] T. Sun, J. M. Kim, G. H. Lee, J. P. Hong, and M. R. Choi, "Effect of pole and slot combination on noise and vibration in permanent magnet synchronous motor," IEEE Transactions on Magnetics, vol. 47, no. 5, pp. 1038-1041, 2011.

[53] J. F. Gieras, "Analytical approach to cogging torque calculation in PM brushless motors," IEMDC 2003 - IEEE International Electric Machines and Drives Conference, vol. 2, no. 5, pp. 815-819, 2003.

[54] F. Magnussen and H. Lendenmann, "Parasitic effects in PM machines with concentrated windings," IEEE Transactions on Industry Applications, vol. 43, no. 5, pp. 1223-1232, 2007.

[55] M. S. Islam, S. Mir, and T. Sebastian, "Issues in reducing the cogging torque of massproduced permanent-magnet brushless DC motor," IEEE Transactions on Industry Applications, vol. 40, no. 3, pp. 813-820, 2004. 
[56] S. Lee, T. Lemley, and G. Keohane, "A comparison study of the commutation methods for the three-phase permanent magnet brushless dc motor," in Electrical Manufacturing Technical Conference 2009: Electrical Manufacturing and Coil Winding Expo, EMCWA 2009, pp. 49-55, 2009.

[57] J. De Viaene, F. Verbelen, S. Derammelaere, and K. Stockman, "Energy-efficient sensorless load angle control of a BLDC motor using sinusoidal currents," IET Electric Power Applications, vol. 12, no. 9, pp. 1378-1389, 2018.

[58] S.-H. Kim, "Vector control of alternating current motors," in Electric Motor Control, pp. 203-246, 2017.

[59] U. Kafader, "Sensorless Control of Brushless DC Motors." Drive.tech by Maxon https://drive.tech/en/stream-content/sensorless-control-of-brushless-motors.

[60] J. C. Gamazo-Real, E. Vázquez-Sánchez, and J. Gómez-Gil, "Position and speed control of brushless dc motors using sensorless techniques and application trends," Sensors, vol. 10, no. 7, pp. 6901-6947, 2010.

[61] P. P. Acarnley and J. F. Watson, "Review of position-sensorless operation of brushless permanent-magnet machines," IEEE Transactions on Industrial Electronics, vol. 53, no. 2, pp. 352-362, 2006.

[62] H. Yang and Y. Chen, "Influence of radial force harmonics with low mode number on electromagnetic vibration of PMSM," IEEE Transactions on Energy Conversion, vol. 29, no. 1, pp. 38-45, 2014.

[63] M. S. Islam, R. Islam, and T. Sebastian, "Noise and vibration characteristics of permanent-magnet synchronous motors using electromagnetic and structural analyses," IEEE Transactions on Industry Applications, vol. 50, no. 5, pp. 3214-3222, 2014.

[64] H. J. Lee, S. U. Chung, and S. M. Hwang, "Noise source identification of a BLDC motor," Journal of Mechanical Science and Technology, vol. 22, no. 4, pp. 708-713, 2008.

[65] R. Shanthi, S. Kalyani, and R. Thangasankaran, "Performance analysis of speed control of PMSM drive with sinusoidal PWM and space vector pwm fed voltage source inverters," IEEE International Conference on Innovations in Green Energy and Healthcare Technologies - 2017, IGEHT 2017, pp. 1-10, 2017.

[66] E. Zeze and K. Akatsu, "Research on vibration analysis and noise-reduction technique of PM motor," Proceedings - 2012 20th International Conference on Electrical Machines, ICEM 2012, pp. 458-463, 2012.

[67] T. Hara, T. Ajima, Y. Tanabe, M. Watanabe, K. Hoshino, and K. Oyama, "Analysis of Vibration and Noise in Permanent Magnet Synchronous Motors with Distributed Winding for the PWM Method," IEEE Transactions on Industry Applications, vol. 54, no. 6, pp. 6042-6049, 2018.

[68] I. P. Tsoumas, H. Tischmacher, and B. Eichinger, "Influence of the number of pole pairs on the audible noise of inverter-fed induction motors: Radial force waves and 
mechanical resonances," Proceedings - 2014 International Conference on Electrical Machines, ICEM 2014, pp. 1811-1817, 2014.

[69] Lo, WC, Chan, CC, Zhu, ZQ, Xu, Howe, Chau, and KT, "Acoustic noise radiated by PWM-controlled induction machine drives," 2000.

[70] J. Le Besnerais, V. Lanfranchi, M. Hecquet, and P. Brochet, "Characterization and reduction of audible magnetic noise due to PWM supply in induction machines," IEEE Transactions on Industrial Electronics, vol. 57, no. 4, pp. 1288-1295, 2010.

[71] T. G. Habetler and D. M. Divan, "Acoustic Noise Reduction in Sinusoidal PWM Drives Using a Randomly Modulated Carrier," Tech. Rep. 3, 1991.

[72] H. V. Trees, "Synthesis of Linear Arrays and Aperatures," in Optimum Array Processing: Part IV of Detection, Estimation, and Modulation Theory, pp. 90-230, John Wiley \& Sons, Inc., 2002.

[73] A. B. Baggeroer and H. Cox, "Passive sonar limits upon nulling multiple moving ships with large aperture arrays," in Conference Record of the Thirty-Third Asilomar Conference on Signals, Systems, and Computers (Cat. No. CH37020), vol. 1, pp. 103108, IEEE, 1999.

[74] V. Oppenheim, "Power Spectral Density," MIT OpenCourseWare, pp. 183-195, 2010.

[75] J. C. Kinsey, R. Eustice, and L. L. Whitcomb, "A Survey of Underwater Vehicle Navigation: Recent Advances and New Challenges," IFAC Conference of Manoeuvring and Control of Marine Craft (MCMC 2006), vol. 88, pp. 1-12, 2006.

[76] B. P. Gibbs, "Advanced Kalman Filtering, Least-Squares and Modeling: A Practical Handbook," Advanced Kalman Filtering, Least-Squares and Modeling: A Practical Handbook, no. c, 2011.

[77] S. J. Julier and J. K. Uhlmann, "New extension of the Kalman filter to nonlinear systems," Signal Processing, Sensor Fusion, and Target Recognition VI, vol. 3068, no. July 1997, p. 182, 1997.

[78] J. Luo, Y. Han, and L. Fan, "Underwater acoustic target tracking: A review," Sensors (Switzerland), vol. 18, no. 1, 2018.

[79] A. Doucet and A. M. Johansen, "A Tutorial on Particle filtering and smoothing: Fiteen years later," The Oxford handbook of nonlinear filtering, no. December 2008, pp. 656-705, 2011.

[80] X. Li, Z. D. Deng, L. T. Rauchenstein, and T. J. Carlson, "Contributed Review: Source-localization algorithms and applications using time of arrival and time difference of arrival measurements," Review of Scientific Instruments, vol. 87, no. 4, 2016.

[81] S. S. Blackman and S. H. Roszkowski, "Application of IMM filtering to passive ranging," SPIE's International Symposium on Optical Science, Engineering, and Instrumentation, vol. 3809, no. October 1999, pp. 270-281, 1999. 
[82] T. Kirubarajan, Y. Bar-Shalom, and D. Lerro, "Bearings-only tracking of maneuvering targets using a batch-recursive estimator," IEEE Transactions on Aerospace and Electronic Systems, vol. 37, no. 3, pp. 770-780, 2001.

[83] M. S. Arulampalam, B. Ristic, N. Gordon, and T. Mansell, "Bearings-only tracking of manoeuvring targets using particle filters," Eurasip Journal on Applied Signal Processing, vol. 2004, no. 15, pp. 2351-2365, 2004.

[84] J.-P. Le Cadre and O. Tremois, "Bearings-Only Tracking for Maneuvering Sources," IEEE Transactions on Aerospace and Electronic Systems, vol. 34, no. 1, pp. 179-193, 1998.

[85] J. Kim, T. Suh, and J. Ryu, "Bearings-only target motion analysis of a highly manoeuvring target," IET Radar, Sonar \& Navigation, vol. 11, no. 6, pp. 1011-1019, 2017.

[86] S. S. Blackman and R. Popoli, Design and analysis of modern tracking systems. 1999.

[87] A. S. Gadre, D. K. Maczka, D. Spinello, B. R. McCarter, D. J. Stilwell, W. Neu, M. J. Roan, and J. B. Hennage, "Cooperative localization of an acoustic using towed hydrophone arrays," 2008 IEEE/OES Autonomous Underwater Vehicles, AUV 2008, 2008.

[88] D. P. Eickstedt and M. R. Benjamin, "Cooperative target tracking in a distributed autonomous sensor network," in OCEANS 2006, pp. 1-6, IEEE, 2006.

[89] H. Roh, M.-H. Cho, and M.-J. Tahk, "Trajectory Optimization Using Cramér-Rao Lower Bound for Bearings-Only Target Tracking," 2018.

[90] J. Le Cadre, "Optimization of the Observer Motion for Bearings-Only Target Motion Analysis," no. December, pp. 3126-3131, 1997.

[91] S. E. Hammel, "Optimal Observer Motion for Bearings-only Localization and Tracking," 1988.

[92] J. M. Passerieux and D. Van Cappel, "Optimal observer maneuver for bearingsonly tracking," Bayesian Bounds for Parameter Estimation and Nonlinear Filtering/Tracking, vol. 34, no. 3, pp. 761-772, 2007.

[93] Y. Oshman and P. Davidson, "Optimization of observer trajectories for bearings-only target localization," IEEE Transactions on Aerospace and Electronic Systems, vol. 35, no. 3, pp. 892-902, 1999.

[94] A. Wolek, B. R. Dzikowicz, J. Mcmahon, and B. H. Houston, "At sea Evaluation of an Underwater Vehicle Beahvior for Passive Target Tracking," pp. 1-10, 2018.

[95] D. Laneuville and C. Jauffret, "Recursive bearings-only TMA via Unscented Kalman Filter: Cartesian vs. modified polar coordinates," IEEE Aerospace Conference Proceedings, vol. 403, pp. 1-11, 2008.

[96] J. M. Passerieux, D. Pillon, P. Blanc-Benon, and C. Jauffret, "Target motion analysis with bearings and frequencies measurements via instrumental variable estimator," in ICASSP, IEEE International Conference on Acoustics, Speech and Signal Processing - Proceedings, vol. 4, pp. 2645-2648, 1989. 
[97] N. H. Nguyen and K. I. DoÊĞGançay, "Single-platform passive emitter localization with bearing and Doppler-shift measurements using pseudolinear estimation techniques," Signal Processing, vol. 125, pp. 336-348, 2016.

[98] K. C. Ho and Y. T. Chan, "An asymptotically unbiased estimator for Bearings-only and Doppler-bearing target motion analysis," IEEE Transactions on Signal Processing, vol. 54, no. 3, pp. 809-822, 2006.

[99] Y. Chan and S. Rudnicki, "Bearings-only and Doppler-bearing tracking using instrumental variables," IEEE Transactions on Aerospace and Electronic Systems, vol. 28, no. 4, pp. 1076-1083, 1992.

[100] S. K. Rao, "Doppler-bearing passive target tracking using a parameterized unscented kalman filter," IETE Journal of Research, vol. 56, no. 1, pp. 69-75, 2010.

[101] X. Li, C. Zhao, J. Yu, and W. Wei, "Underwater bearing-only and bearing-doppler target tracking based on square root unscented Kalman filter," Entropy, vol. 21, no. 8, 2019 .

[102] C. Jauffret and A. C. Pérez, "Efficient Pseudolinear Estimation for Bearings and Frequencies Target Motion Analysis," 2018 21st International Conference on Information Fusion, FUSION 2018, pp. 2556-2563, 2018.

[103] B. Ristic and A. Farina, "Target tracking via multi-static Doppler shifts," IET Radar, Sonar \& Navigation, vol. 7, no. 5, pp. 508-516, 2013.

[104] I. Shames, A. N. Bishop, M. Smith, and B. D. Anderson, "Doppler shift target localization," IEEE Transactions on Aerospace and Electronic Systems, vol. 49, no. 1, pp. 266-276, 2013.

[105] B. Southall, B. F. Buxton, and J. A. Marchant, "Controllability and Observability: Tools for Kalman Filter Design," pp. 17.1-17.10, 2013.

[106] R. van der Merwe and E. Wan, "Sigma-Point Kalman Filters for Probabilistic Inference in Dynamic State-Space Models," Proceedings of the Workshop on Advances in Machine Learning, no. April, 2003.

[107] C. J. McFarland, M. V. Jakuba, S. Suman, J. C. Kinsey, and L. L. Whitcomb, "Toward ice-relative navigation of underwater robotic vehicles under moving sea ice: Experimental evaluation in the Arctic sea," Proceedings - IEEE International Conference on Robotics and Automation, vol. 2015-June, no. June, pp. 1527-1534, 2015.

[108] J. C. Kinsey, D. R. Yoerger, M. V. Jakuba, R. Camilli, C. R. Fisher, and C. R. German, "Assessing the deepwater horizon oil spill with the sentry autonomous underwater vehicle," IEEE International Conference on Intelligent Robots and Systems, pp. 261-267, 2011.

[109] P. F. J. Lermusiaux, T. Lolla, P. J. H. Jr, K. Yigit, M. P. Ueckermann, T. Sondergaard, and W. G. Leslie, "Science of Autonomy: Time-Optimal Path Planning and Adaptive Sampling for Swarms of Ocean Vehicles," in Handbook of Ocean Engineering, pp. 481497, 2016. 
[110] M. Eckstein, "DARPA, BAE Systems Developing Small Unmanned Underwater Vehicles to Hunt Enemy Submarines," US Naval Institute News, pp. 1-8, 2017.

[111] "Large Displacement Unmanned Underwater Vehicle Innovative Naval Prototype Technology," Office of Naval Research ONR BAA Announcement 11-025, 2011.

[112] M. Eckstein, "Navy Hosts Virtual Industry Day to Keep Hammerhead Mine on Accelerated Acquisition Path," US Naval Institute News, pp. 1-10, 2020.

[113] G. L. Ogden, L. M. Zurk, M. E. Jones, and M. E. Peterson, "Extraction of small boat harmonic signatures from passive sonar," The Journal of the Acoustical Society of America, vol. 129, no. 107, 2011.

[114] W. C. Dixon and C. R. Rollins, "Very Low Frequency Acoustic Detection of Submarines," Naval Research Laboratory, 1977.

[115] S. Stanic, "Passive Swimmer Detection Passive Acoustic Ranging by Multimode Waveguide," pp. 97-98, 2004.

[116] L. Fillinger, P. De Theije, M. Zampolli, A. Sutin, H. Salloum, N. Sedunov, and A. Sedunov, "Towards a passive acoustic underwater system for protecting harbours against intruders," 2010 International Waterside Security Conference, WSS 2010, 2010.

[117] A. Sutin, B. Bunin, A. Sedunov, N. Sedunov, L. Fillinger, M. Tsionskiy, and M. Bruno, "Stevens passive acoustic system for underwater surveillance," 2010 International Waterside Security Conference, WSS 2010, 2010.

[118] N. N. D. Moura, J. M. D. Seixas, and R. Ramos, "Passive Sonar Signal Detection and Classification Based on Independent Component Analysis," Sonar Systems, pp. 93$103,2011$.

[119] J. D. Holmes, W. M. Careyjames, and F. Lynch, "An overview of unmanned underwater vehicle noise in the low to mid frequencies bands," Acoustical Society of America, vol. 9, 2010.

[120] J. Gebbie, M. Siderius, and J. S. Allen Iii, "Aspect-dependent radiated noise analysis of an underway autonomous underwater vehicle," Journal of Acoustical Society of America, 2012.

[121] S. Zhang and X. Ma, "A speed measurement method using AUV radiated noise spectrum," in 2018 OCEANS - MTS/IEEE Kobe Techno-Oceans, OCEANS - Kobe 2018, 2018.

[122] D. G. Dorrell, M. F. Hsieh, M. Popescu, L. Evans, D. A. Staton, and V. Grout, "A review of the design issues and techniques for radial-flux brushless surface and internal rare-earth permanent-magnet motors," IEEE Transactions on Industrial Electronics, vol. 58, no. 9, pp. 3741-3757, 2011.

[123] A. Lelkes, J. Krotsch, and R. W. D. Doncker, "Low-Noise External Rotor BLDC Motor for Fan Applications," Conference Record of the 2002 IEEE Industry Applications Conference. 37th IAS Annual Meeting (Cat. No.02CH37344), vol. 3, pp. 2036-2042 vol.3, 2002. 
[124] P. D. Welch, "The Use of Fast Fourier Transform for the Estimation of Power Spectra: A Method Based on Time Averaging Over Short, Modified Periodograms," IEEE Transactions on Audio and Electroacoustics, vol. AU-15, no. 2, pp. 70-73, 1967.

[125] B. Allotta, R. Costanzi, F. Fanelli, N. Monni, L. Paolucci, and A. Ridolfi, "Sea currents estimation during AUV navigation using Unscented Kalman Filter," IFACPapersOnLine, vol. 50, no. 1, pp. 13668-13673, 2017.

[126] S. A. Randeni P., A. L. Forrest, R. Cossu, Z. Q. Leong, and D. Ranmuthugala, "Determining the horizontal and vertical water velocity components of a turbulentwater column using the motion response of an autonomous underwater vehicle," Journal of Marine Science and Engineering, vol. 5, no. 3, 2017.

[127] Ø. Hegrenaes and O. Hallingstad, "Model-aided INS with sea current estimation for robust underwater navigation," IEEE Journal of Oceanic Engineering, vol. 36, no. 2, pp. 316-337, 2011.

[128] B. Borowski, A. Sutin, H.-S. Roh, and B. Bunin, "Passive acoustic threat detection in estuarine environments," International Society for Optics and Photonics, vol. 6945, no. Optics and Photonics in Global Homeland Security IV, 2008.

[129] Z. Y. Zhang, "Diver Detection Sonars and Target Strength: Review and Discussions," 14 th International Congress on Sound ES Vibration, pp. 1-8, 2007.

[130] L. Fillinger, A. J. Hunter, M. Zampolli, M. C. Clarijs, and . A. J. Hunter, "Passive acoustic detection of closed-circuit underwater breathing apparatus in an operational port environment," The Journal of the Acoustical Society of America, vol. 132, no. 10, pp. 700671121-14800503, 2012.

[131] M. Bruno, K. W. Chung, H. Salloum, A. Sedunov, N. Sedunov, A. Sutin, H. Graber, and P. Mallas, "Concurrent use of satellite imaging and passive acoustics for maritime domain awareness," 2010 International Waterside Security Conference, WSS 2010, pp. $1-8,2010$.

[132] A. J. Poulsen, D. P. Eickstedt, and J. Ianniello, "Bearing Stabilization and Tracking for an AUV with an Acoustic Line Array," IEEE Oceans, 2006.

[133] M. J. Hamilton, S. Kemna, and D. Hughes, "Antisubmarine Warfare Applications for Autonomous Underwater Vehicles: The GLINT09 Sea Trial Results," Journal of Field Robotics, 2010.

[134] G. Ferri, A. Munafò, R. Goldhahn, and K. Lepage, "Results from COLLAB13 sea trial on tracking underwater targets with AUVs in bistatic sonar scenarios," 2014 Oceans - St. John's, OCEANS 2014, 2015.

[135] G. Ferri, A. Munafo, and K. D. LePage, "An Autonomous Underwater Vehicle DataDriven Control Strategy for Target Tracking," IEEE Journal of Oceanic Engineering, vol. 43, no. 2, pp. 323-343, 2018.

[136] H. Schmidt, M. R. Benjamin, S. M. Petillo, and R. Lum, "Nested autonomy for distributed ocean sensing," in Springer Handbook of Ocean Engineering, pp. 459-479, 2016. 
[137] C. Allen and C. Allen, "20,000 Drones Under the Sea," U.S. Naval Institute, vol. $144 / 7 / 1,385,2018$.

[138] C. Woody, "Watch the US Coast Guard seize a narco sub laden with more than 5,600 pounds of cocaine," Business Insider, 2016.

[139] M. L. Barlett and G. R. Wilson, "Characteristics of Small Boat Signatures," Journal of Acoustical Society of America, 2002.

[140] C. Erbe, S. Liong, M. W. Koessler, A. J. Duncan, and T. Gourlay, "Underwater sound of rigid-hulled inflatable boats," The Journal of the Acoustical Society of America, vol. 139, no. 6, pp. EL223-EL227, 2016.

[141] J. Cuschieri and F. Debiesme, "Characterizing the Sources of Noise and Vibration on a Small Autonomous Submersible," International Congress and Exhibition on Noise Control Engineering, no. August, pp. 1-6, 2000.

[142] R. Zimmerman, G. L. D'Spain, and C. D. Chadwell, "Decreasing the radiated acoustic and vibration noise of a mid-size AUV," IEEE Journal of Oceanic Engineering, vol. 30, no. 1, pp. 179-187, 2005.

[143] W. A. Salah, D. Ishak, and K. J. Hammadi, "PWM switching strategy for torque ripple minimization in BLDC motor," Journal of Electrical Engineering, vol. 62, no. 3, pp. 141-146, 2011.

[144] P. S. A. Randeni, N. R. Rypkema, E. M. Fischell, A. L. Forrest, M. R. Benjamin, and H. Schmidt, "Implementation of a Hydrodynamic Model-Based Navigation System for a Low-Cost AUV Fleet," in AUV 2018 - 2018 IEEE/OES Autonomous Underwater Vehicle Workshop, Proceedings, 2018.

[145] R. P. Hodges, "Underwater Acoustics: Analysis, Design and Performance of Sonar," Underwater Acoustic Systems, pp. 16-31, 1990.

[146] S. Atapattu, C. Tellambura, and H. Jiang, "Analysis of area under the ROC curve of energy detection," IEEE Transactions on Wireless Communications, vol. 9, no. 3, pp. 1216-1225, 2010.

[147] H. V. Trees, "Arrays and Spatial Filters," in Optimum Array Processing: Part IV of Detection, Estimation, and Modulation Theory, ch. 2, pp. 17-89, John Wiley \& Sons, Inc., 2002.

[148] E. Gentilho, P. R. Scalassara, and T. Abrão, "Direction-of-Arrival Estimation Methods: A Performance-Complexity Tradeoff Perspective," Journal of Signal Processing Systems, vol. 92, no. 2, pp. 239-256, 2020.

[149] J.-P. Marage and Y. Mori, "Basic Theoretical Notions in Active Processing," Sonar and Underwater Acoustics, pp. 459-524, 2013.

[150] F. B. Jensen, W. A. Kuperman, M. B. Porter, and H. Schmidt, Computational Ocean Acoustics. Woodbury: American Institute of Physics, 1994.

[151] M. B. Porter, "The BELLHOP Manual and User's Guide (Preliminary Draft)," pp. 157, 2011. 
[152] O. C. Rodriguez, "General description of the Bellhop ray tracing program," 2008.

[153] W. A. Kuperman and P. Roux, "Underwater acoustics," in Springer Handbook of Acoustics, pp. 157-212, Springer, 2014.

[154] G.-C. Hsieh and J. C. Hung, "Phase-Locked Loop Techniques-A Survey," IEEE Transactions on Industrial Electronics, vol. 43, no. 6, pp. 609-615, 1996.

[155] H. L. V. Trees, "Adaptive Beamformers," in Optimum Array Processing: Part IV of Detection, Estimation, and Modulation Theory, vol. 4, pp. 710-916, 2002.

[156] M. Zhang, W. Xu, and Y. Xu, "Inversion of the Sound Speed with Radiated Noise of an Autonomous Underwater Vehicle in Shallow Water Waveguides," IEEE Journal of Oceanic Engineering, vol. 41, no. 1, pp. 204-216, 2016.

[157] Benjamin Vedder, "VESC - Open Source ESC," 2015. http://vedder.se/2015/01/vesc-open-source-esc/. 